\title{
Direct Observation of Aminyl Radical Intermediate During Single Crystal to Single Crystal Photoinduced Orton Rearrangement
}

Panče Naumov, Kenji Sakurai, Masahiko Tanaka, Hideyuki Hara

\section{SUPPORTING INFORMATION}




\section{FIGURES}
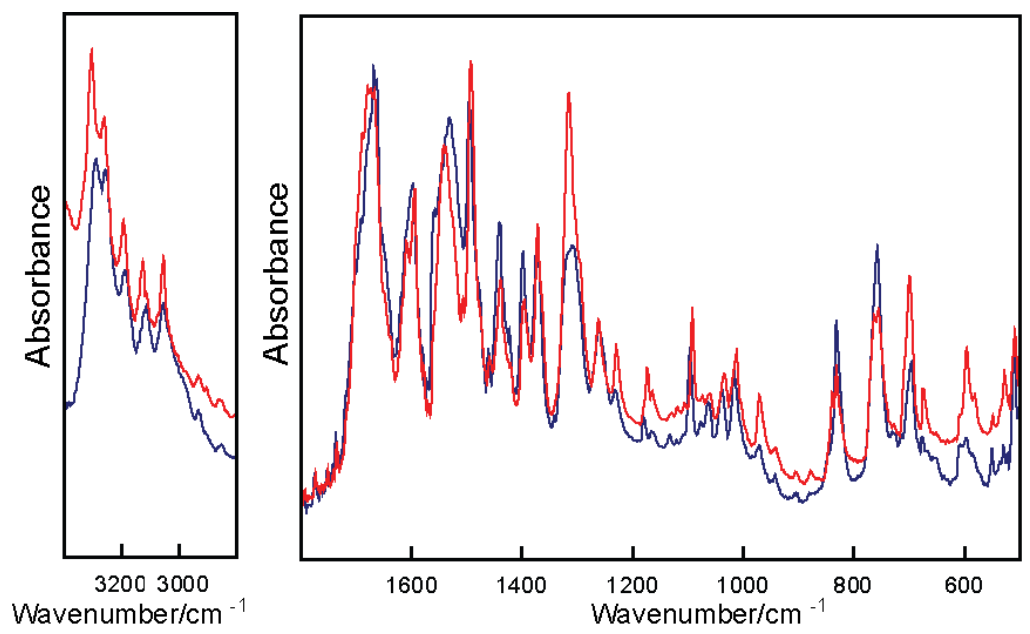

Figure S1. FTIR spectra of ex situ (red) and in situ (blue) CAAB UV-irradiated (365 nm) in $\mathrm{KBr}$ pellets. The spectra show that the $\mathrm{KBr}$ matrix does not have significant influence on the irradiation product $(p-\mathrm{CA})$. 


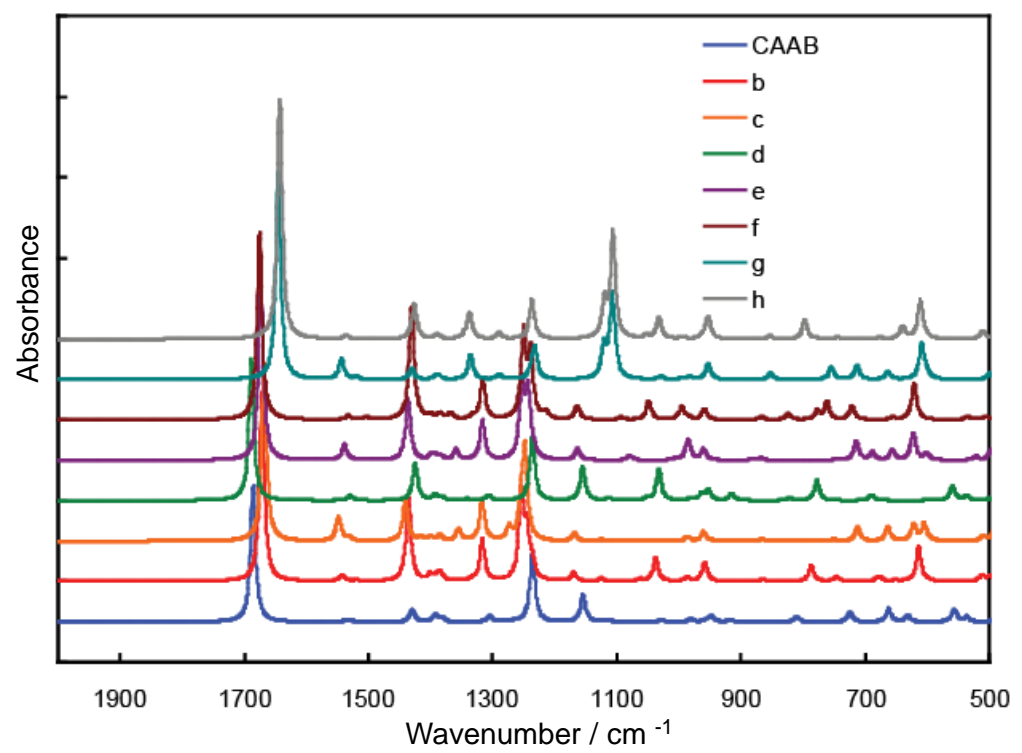<smiles>CC(=O)N(Cl)c1ccccc1</smiles>

a<smiles>CC(=O)Nc1ccc(Cl)cc1</smiles>

b<smiles>CC(=O)Nc1ccccc1</smiles>

C<smiles>CC(=O)N(Cl)c1ccc(Cl)cc1</smiles>

d<smiles>CC(=O)Nc1ccccc1Cl</smiles>

e<smiles>CC(=O)Nc1ccc(Cl)cc1Cl</smiles>

$\mathbf{f}$<smiles>C/C(O)=N\c1ccccc1</smiles>

g<smiles>C/C(O)=N\c1ccc(Cl)cc1</smiles>

$\mathbf{h}$

Figure S2. Theoretical harmonic IR spectra (top) of CAAB, $p$-CA and six models of the photolysis products (bottom) calculated at B3LYP/6-31G(d,p) level. The vibrational frequencies were uniformly scaled with $f=0.9323$, the factor obtained from the experimental (solid state) and theoretical (single molecule) frequency of the $v(C=O)$ mode of CAAB. 


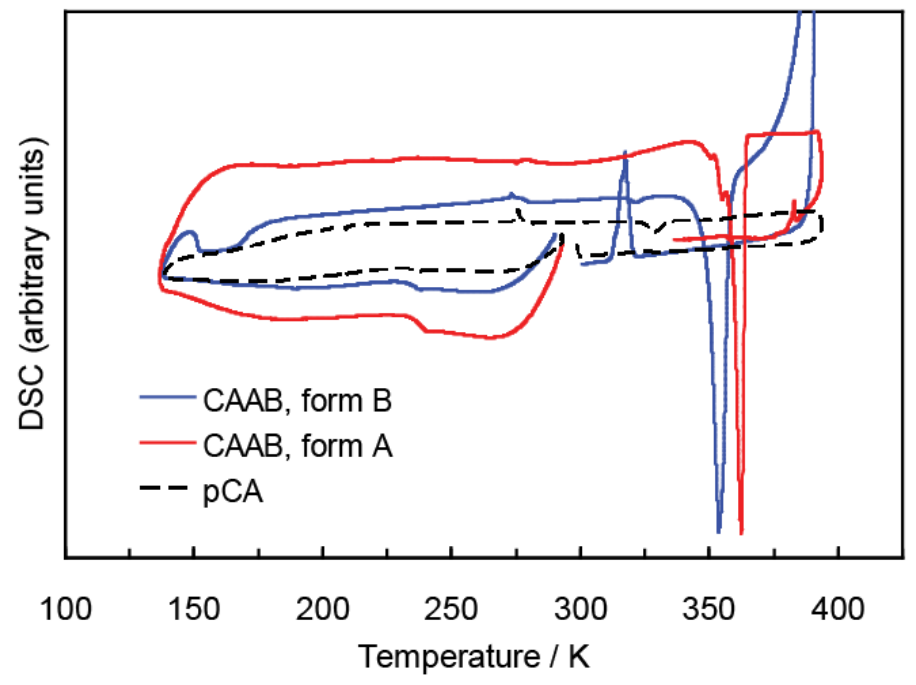

Figure S3. Differential scanning calorimetry (DSC) curves recorded by cooling and subsequent heating of the polymorphic modifications of $\mathrm{CAAB}, \mathrm{A}$ and $\mathrm{B}$, and parachloroacetanilide ( $\mathrm{pCA}$ ) (pure pCA was obtained by recrystallization of CAAB from $\mathrm{CHCl}_{3}$ ).

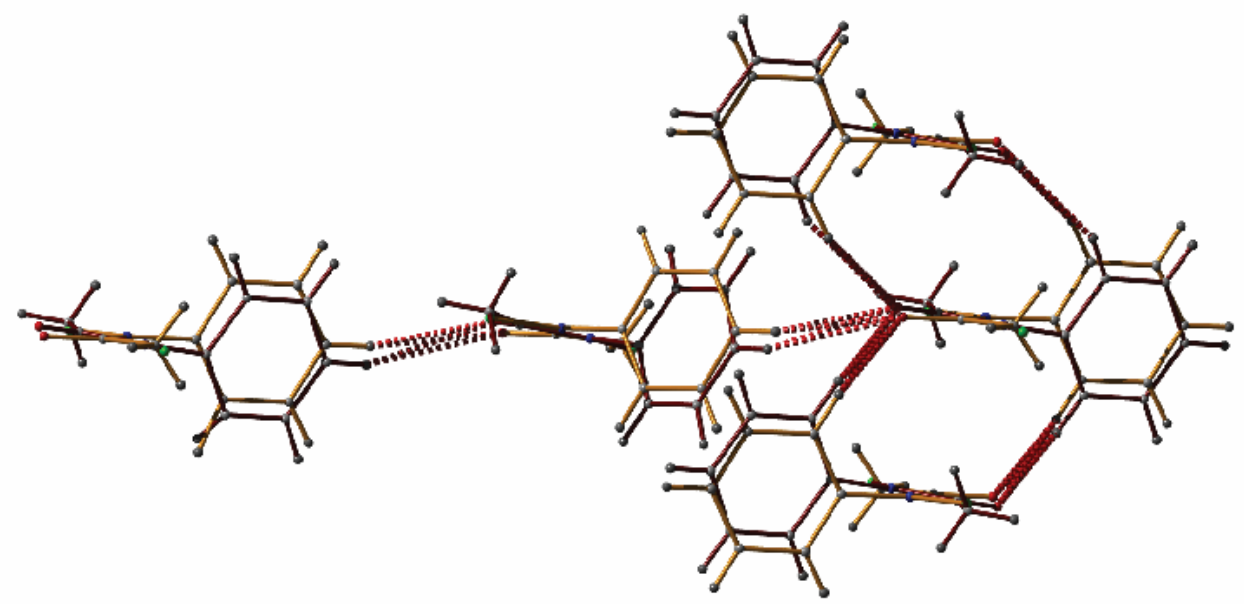

Figure S4. Part of the packing motif in the thermally disordered, non-reacted CAAB crystal showing the relevant $\mathrm{C}-\mathrm{H} \cdots \mathrm{O}$ hydrogen bonds. 


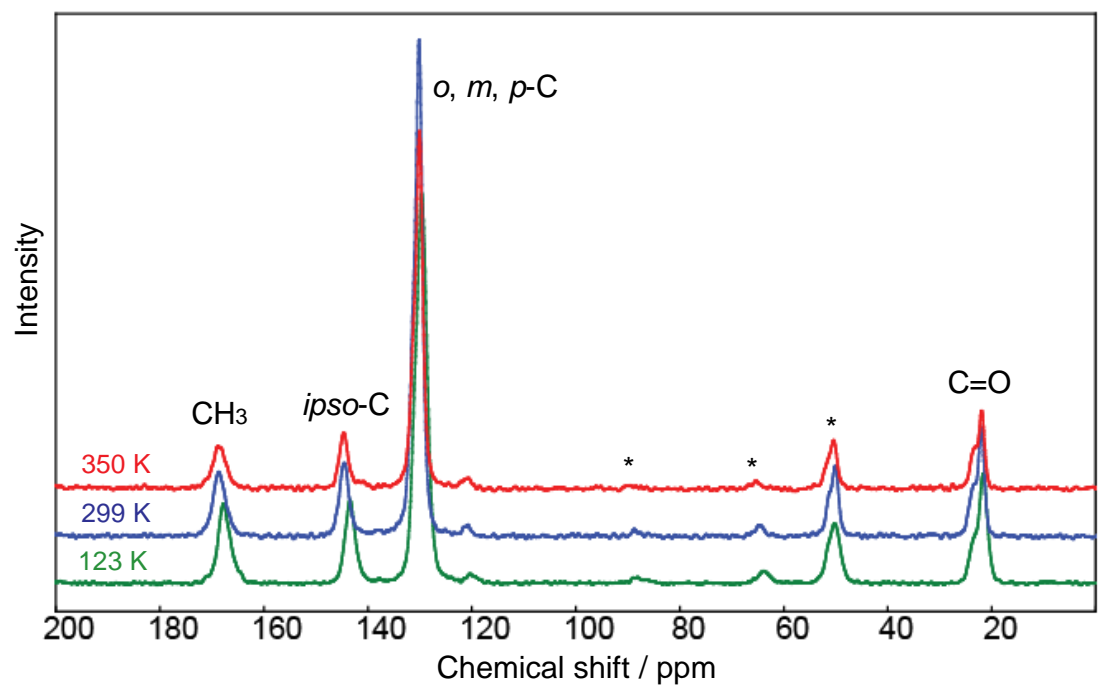

Figure S5. Variable-temperature ${ }^{13} \mathrm{CP}$ MAS NMR spectra of non-irradiated CAAB. The spinning side-bands are marked with asterisks. The NMR assignments are based on the SCF GIAO shielding tensors calculated for the B3LYP/6-31G(d,p) optimized structure of CAAB.

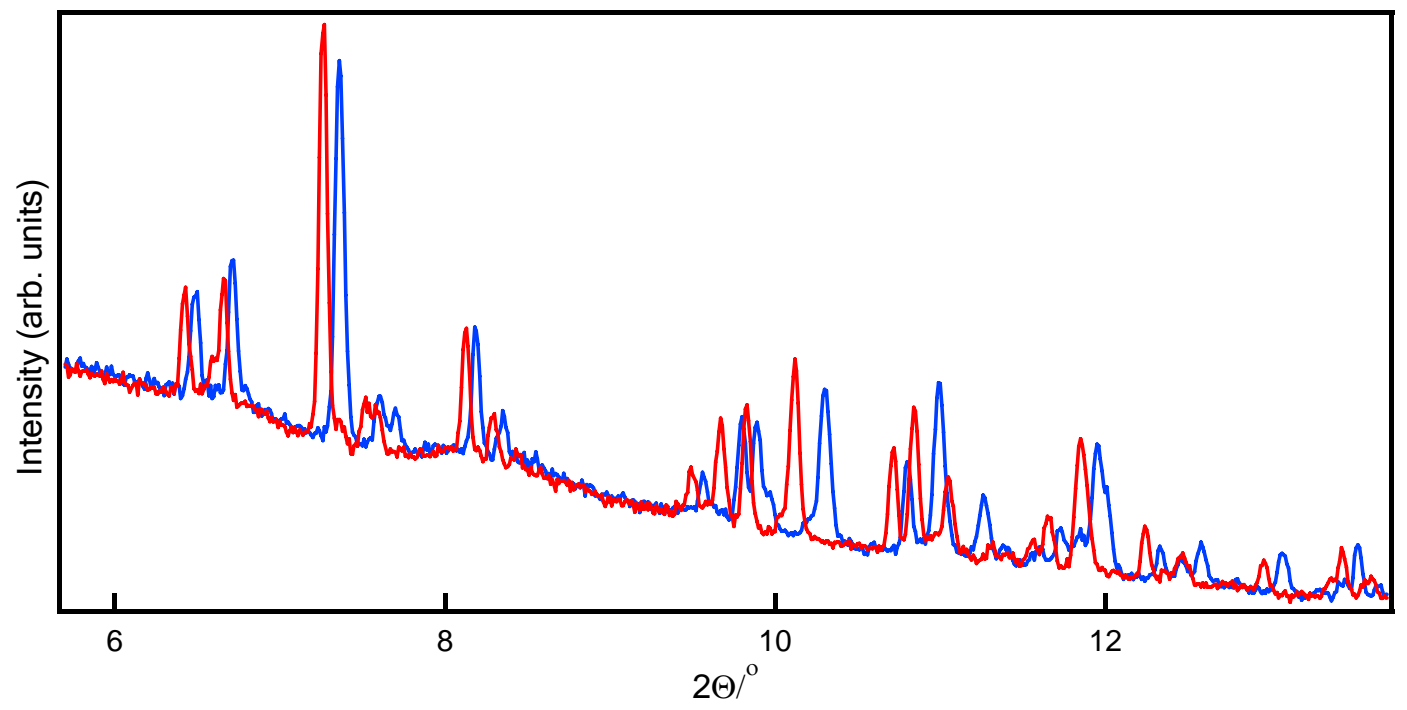

Figure S6. Thermally induced changes of the low-angle region in the powder patterns of non-irradiated $\mathrm{CAAB}$ recorded at $200 \mathrm{~K}$ (blue line) and $300 \mathrm{~K}$ (red line) using synchrotron $\mathrm{X}$-ray radiation $(\lambda=0.6358 \AA$, background not subtracted). 

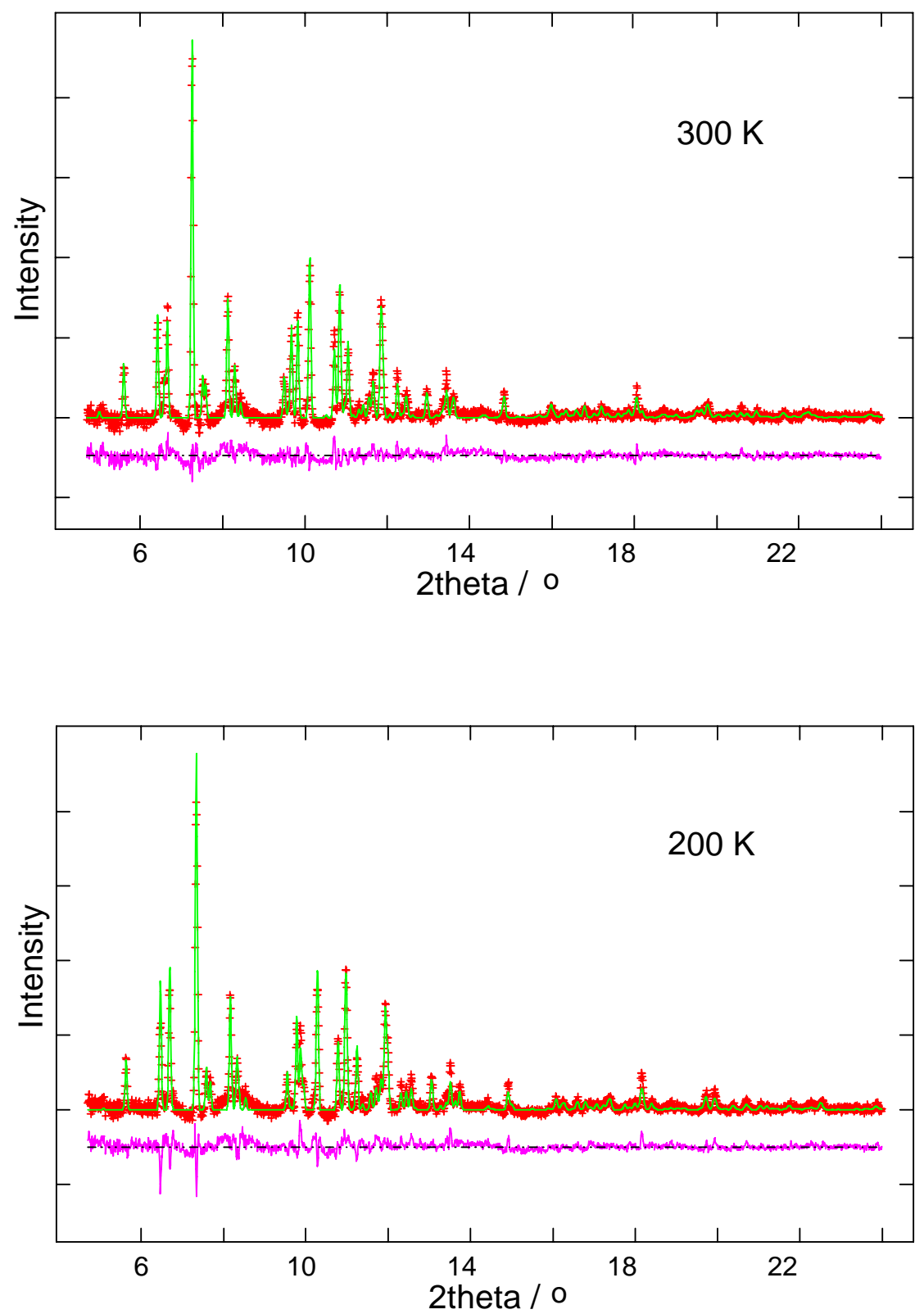

Figure S7. Experimental (red crosses) and simulated (green line) synchrotron powder Xray diffraction patterns $(\lambda=0.6358 \AA$, background subtracted), and their difference (pink line) of doubly disordered $\mathrm{CAAB}$ in non-irradiated microcrystalline powder. 


\section{TABLES}

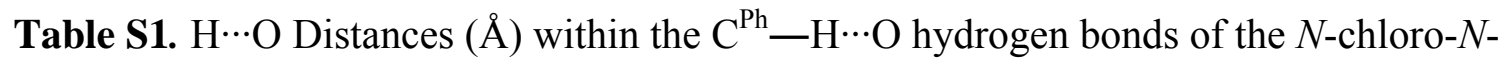
acetyl group

\begin{tabular}{cccc}
\hline Residue \# & Acceptor & \multicolumn{2}{c}{ Donor } \\
\cline { 3 - 4 } & & $\mathrm{O} 1$ & $\mathrm{O}^{\prime}$ \\
\hline $\mathrm{Ph} 1^{[\mathrm{a}]}$ & $\mathrm{C} 6$ (ortho) & 2.615 & 2.983 \\
& $\mathrm{C} 6^{\prime}$ (ortho) & 2.230 & 2.585 \\
\hline $\mathrm{Ph} 2^{[\mathrm{b}]}$ & $\mathrm{C} 2$ (ortho) & 2.446 & 2.372 \\
& $\mathrm{C} 2^{\prime}$ (ortho) & 2.863 & 2.664 \\
$\mathrm{Ph} 3^{[\mathrm{c}]}$ & $\mathrm{C} 4$ (para) & 2.695 & 2.582 \\
& $\mathrm{C} 4{ }^{\prime}$ (para) & 2.852 & 2.835
\end{tabular}

Symmetry operators: [a] $x, y, z[\mathrm{~b}] 1+x, 1-y,-z[\mathrm{c}] 1-x, 0.5+y, 0.5-z$.

Table S2. Molecular models, Cartesian coordinates and intramolecular parameters of the optimized structures of the model compounds (theoretical level: B3LYP/6-31G(d,p), the labels refer to Fig. S2)

\section{S2.1. CAAB}

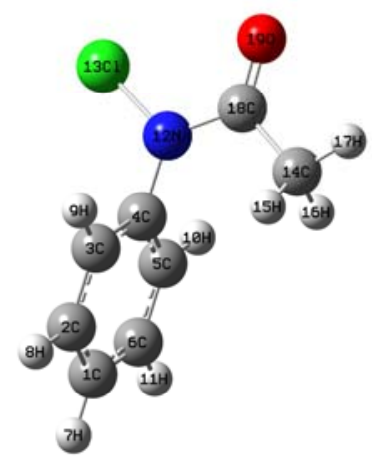

OPTIMIZED PARAMETERS

\begin{tabular}{|c|c|c|c|}
\hline R1 & $\mathrm{R}(1,2)$ & 1.3966 & $-\mathrm{DE} / \mathrm{DX}=$ \\
\hline R2 & $\mathrm{R}(1,6)$ & 1.3967 & $-\mathrm{DE} / \mathrm{DX}=$ \\
\hline R3 & $\mathrm{R}(1,7)$ & 1.0858 & $-\mathrm{DE} / \mathrm{DX}=$ \\
\hline R4 & $\mathrm{R}(2,3)$ & 1.3943 & $-\mathrm{DE} / \mathrm{DX}=$ \\
\hline R5 & $\mathrm{R}(2,8)$ & 1.0856 & $-\mathrm{DE} / \mathrm{DX}=$ \\
\hline R6 & $\mathrm{R}(3,4)$ & 1.3989 & $-\mathrm{DE} / \mathrm{DX}=$ \\
\hline R7 & $\mathrm{R}(3,9)$ & 1.0852 & $-\mathrm{DE} / \mathrm{DX}=$ \\
\hline
\end{tabular}

\begin{tabular}{|c|c|c|c|}
\hline R8 & $\mathrm{R}(4,5)$ & 1.3995 & $-\mathrm{DE} / \mathrm{DX}=$ \\
\hline R9 & $\mathrm{R}(4,12)$ & 1.4301 & $-\mathrm{DE} / \mathrm{DX}=$ \\
\hline R10 & $\mathrm{R}(5,6)$ & 1.3942 & $-\mathrm{DE} / \mathrm{DX}=$ \\
\hline R11 & $\mathrm{R}(5,10)$ & 1.0855 & $-\mathrm{DE} / \mathrm{DX}=$ \\
\hline R12 & $\mathrm{R}(6,11)$ & 1.0857 & $-\mathrm{DE} / \mathrm{DX}=$ \\
\hline R13 & $\mathrm{R}(12,13)$ & 1.7506 & $-\mathrm{DE} / \mathrm{DX}=$ \\
\hline R14 & $\mathrm{R}(12,18)$ & 1.3939 & $-\mathrm{DE} / \mathrm{DX}=$ \\
\hline R15 & $\mathrm{R}(14,15)$ & 1.0925 & $-\mathrm{DE} / \mathrm{DX}=0.0$ \\
\hline R16 & $\mathrm{R}(14,16)$ & 1.094 & $-\mathrm{DE} / \mathrm{DX}=0.0$ \\
\hline R17 & $\mathrm{R}(14,17)$ & 1.0897 & $-\mathrm{DE} / \mathrm{DX}=0.0$ \\
\hline R18 & $\mathrm{R}(14,18)$ & 1.5231 & $-\mathrm{DE} / \mathrm{DX}=0.0$ \\
\hline R19 & $\mathrm{R}(18,19)$ & 1.214 & $-\mathrm{DE} / \mathrm{DX}=0.0$ \\
\hline A1 & $\mathrm{A}(2,1,6)$ & 120.1361 & $-\mathrm{DE} / \mathrm{DX}=0.0$ \\
\hline A2 & $\mathrm{A}(2,1,7)$ & 119.9355 & $-\mathrm{DE} / \mathrm{DX}=0.0$ \\
\hline A3 & $\mathrm{A}(6,1,7)$ & 119.928 & $-\mathrm{DE} / \mathrm{DX}=0.0$ \\
\hline A4 & $\mathrm{A}(1,2,3)$ & 120.0663 & $-\mathrm{DE} / \mathrm{DX}=0.0$ \\
\hline A5 & $\mathrm{A}(1,2,8)$ & 120.1389 & $-\mathrm{DE} / \mathrm{DX}=$ \\
\hline A6 & $\mathrm{A}(3,2,8)$ & 119.7947 & $-\mathrm{DE} / \mathrm{DX}=$ \\
\hline A7 & $\mathrm{A}(2,3,4)$ & 119.7122 & $-\mathrm{DE} / \mathrm{DX}=$ \\
\hline A8 & $\mathrm{A}(2,3,9)$ & 121.1514 & $-\mathrm{DE} / \mathrm{DX}=$ \\
\hline A9 & $\mathrm{A}(4,3,9)$ & 119.1364 & $-\mathrm{DE} / \mathrm{DX}=0.0$ \\
\hline A10 & $\mathrm{A}(3,4,5)$ & 120.3113 & $-\mathrm{DE} / \mathrm{DX}=0.0$ \\
\hline A11 & $\mathrm{A}(3,4,12)$ & 119.479 & $-\mathrm{DE} / \mathrm{DX}=$ \\
\hline A12 & $\mathrm{A}(5,4,12)$ & 120.2081 & $-\mathrm{DE} / \mathrm{DX}=0.0$ \\
\hline A13 & $\mathrm{A}(4,5,6)$ & 119.7013 & $-\mathrm{DE} / \mathrm{DX}=0.0$ \\
\hline A14 & $\mathrm{A}(4,5,10)$ & 119.3224 & $-\mathrm{DE} / \mathrm{DX}=0.0$ \\
\hline A15 & $\mathrm{A}(6,5,10)$ & 120.9761 & $-\mathrm{DE} / \mathrm{DX}=0.0$ \\
\hline A16 & $\mathrm{A}(1,6,5)$ & 120.0647 & $-\mathrm{DE} / \mathrm{DX}=0.0$ \\
\hline A17 & $\mathrm{A}(1,6,11)$ & 120.1415 & $-\mathrm{DE} / \mathrm{DX}=0.0$ \\
\hline A18 & $\mathrm{A}(5,6,11)$ & 119.7933 & $-\mathrm{DE} / \mathrm{DX}=0.0$ \\
\hline A19 & $\mathrm{A}(4,12,13)$ & 115.6464 & $-\mathrm{DE} / \mathrm{DX}=0.0$ \\
\hline A20 & $\mathrm{A}(4,12,18)$ & 127.139 & $-\mathrm{DE} / \mathrm{DX}=0.0$ \\
\hline A 21 & $\mathrm{~A}(13,12,18)$ & 116.2791 & $-\mathrm{DE} / \mathrm{DX}=0.0$ \\
\hline A22 & $\mathrm{A}(15,14,16)$ & 107.6097 & $-\mathrm{DE} / \mathrm{DX}=0.0$ \\
\hline
\end{tabular}




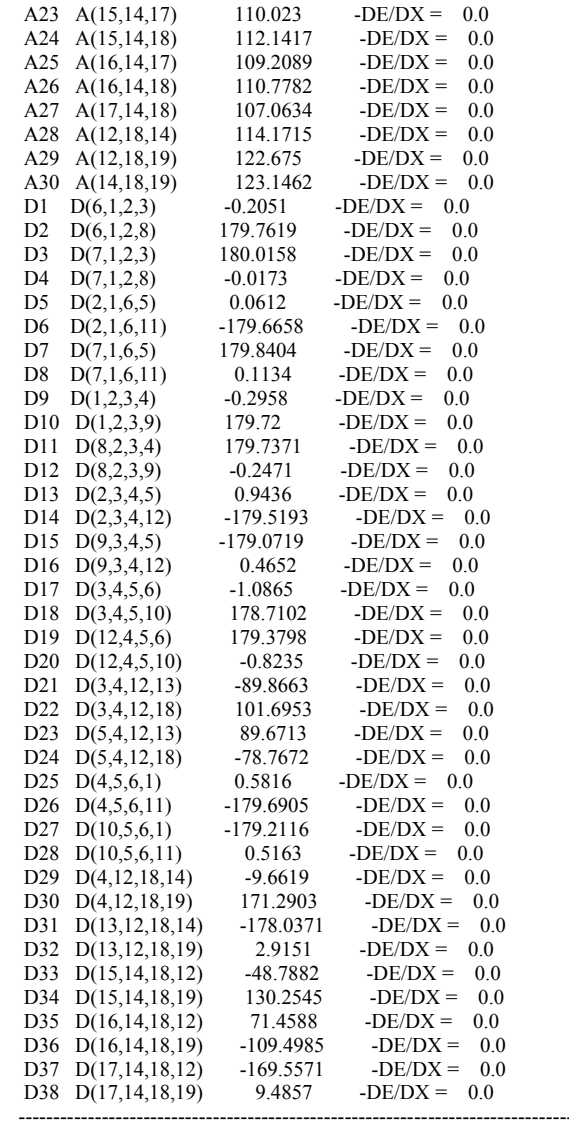

CARTESIAN COORDINATES

$\begin{array}{cccccc}1 & 6 & 0 & -.054936 & .169539 & -.074288 \\ 2 & 6 & 0 & -.027240 & .248395 & 1.319847 \\ 3 & 6 & 0 & 1.179648 & .107508 & 2.003625 \\ 4 & 6 & 0 & 2.359868 & -.119080 & 1.287615 \\ 5 & 6 & 0 & 2.336436 & -.184977 & -.110107 \\ 6 & 6 & 0 & 1.126058 & -.046297 & -.788016 \\ 7 & 1 & 0 & -.995972 & .279819 & -.604653 \\ 8 & 1 & 0 & -.944628 & .420015 & 1.874445 \\ 9 & 1 & 0 & 1.222161 & .170178 & 3.086194 \\ 10 & 1 & 0 & 3.264897 & -.340487 & -.650612 \\ 11 & 1 & 0 & 1.105432 & -.102202 & -1.872048 \\ 12 & 7 & 0 & 3.593415 & -.273143 & 1.994660 \\ 13 & 17 & 0 & 4.490276 & 1.202118 & 2.283929 \\ 14 & 6 & 0 & 3.452166 & -2.718616 & 1.963281 \\ 15 & 1 & 0 & 2.441363 & -2.671376 & 2.375107 \\ 16 & 1 & 0 & 3.362813 & -2.862747 & .882494 \\ 17 & 1 & 0 & 3.991507 & -3.561770 & 2.394029 \\ 18 & 6 & 0 & 4.258746 & -1.465150 & 2.276364 \\ 19 & 8 & 0 & 5.377201 & -1.495950 & 2.747458\end{array}$

S2.2. b

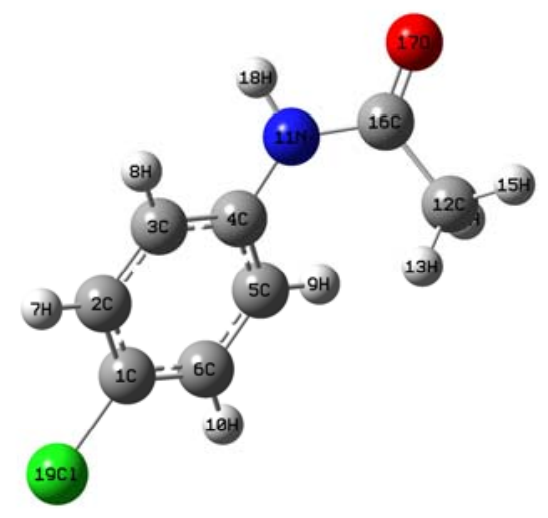

OPTIMIZED PARAMETERS

\begin{tabular}{|c|c|c|c|}
\hline R1 & $\mathrm{R}(1,2)$ & 1.3944 & $-\mathrm{DE} / \mathrm{DX}=$ \\
\hline R2 & $\mathrm{R}(1,6)$ & 1.393 & $-\mathrm{DE} / \mathrm{DX}=0.0$ \\
\hline R3 & $\mathrm{R}(1,19)$ & 1.7576 & $-\mathrm{DE} / \mathrm{DX}=0.0$ \\
\hline R4 & $\mathrm{R}(2,3)$ & 1.3914 & $-\mathrm{DE} / \mathrm{DX}=0.0$ \\
\hline R5 & $\mathrm{R}(2,7)$ & 1.0841 & $-\mathrm{DE} / \mathrm{DX}=$ \\
\hline R6 & $\mathrm{R}(3,4)$ & 1.4037 & $-\mathrm{DE} / \mathrm{DX}=$ \\
\hline R7 & $\mathrm{R}(3,8)$ & 1.0862 & $-\mathrm{DE} / \mathrm{DX}=$ \\
\hline R8 & $\mathrm{R}(4,5)$ & 1.4022 & $-\mathrm{DE} / \mathrm{DX}=0.0$ \\
\hline R9 & $\mathrm{R}(4,11)$ & 1.4108 & $-\mathrm{DE} / \mathrm{DX}=0.0$ \\
\hline R10 & $\mathrm{R}(5,6)$ & 1.3938 & $-\mathrm{DE} / \mathrm{DX}=$ \\
\hline R11 & $\mathrm{R}(5,9)$ & 1.0831 & $-\mathrm{DE} / \mathrm{DX}=$ \\
\hline R12 & $\mathrm{R}(6,10)$ & 1.0842 & $-\mathrm{DE} / \mathrm{DX}=0.0$ \\
\hline R13 & $\mathrm{R}(11,16)$ & 1.3878 & $-\mathrm{DE} / \mathrm{DX}=0.0$ \\
\hline R14 & $\mathrm{R}(11,18)$ & 1.013 & $-\mathrm{DE} / \mathrm{DX}=0.0$ \\
\hline R15 & $\mathrm{R}(12,13)$ & 1.0919 & $-\mathrm{DE} / \mathrm{DX}=0.0$ \\
\hline R16 & $\mathrm{R}(12,14)$ & 1.0952 & $-\mathrm{DE} / \mathrm{DX}=$ \\
\hline R17 & $\mathrm{R}(12,15)$ & 1.0904 & $-\mathrm{DE} / \mathrm{DX}=$ \\
\hline R18 & $\mathrm{R}(12,16)$ & 1.5163 & $-\mathrm{DE} / \mathrm{DX}=$ \\
\hline R19 & $\mathrm{R}(16,17)$ & 1.2217 & $-\mathrm{DE} / \mathrm{DX}=$ \\
\hline $\mathrm{A} 1$ & $\mathrm{~A}(2,1,6)$ & 120.6092 & $-\mathrm{DE} / \mathrm{DX}=0.0$ \\
\hline A2 & $\mathrm{A}(2,1,19)$ & 119.6783 & $-\mathrm{DE} / \mathrm{DX}=0.0$ \\
\hline A3 & $\mathrm{A}(6,1,19)$ & 119.711 & $-\mathrm{DE} / \mathrm{DX}=0.0$ \\
\hline A4 & $\mathrm{A}(1,2,3)$ & 119.3775 & $-\mathrm{DE} / \mathrm{DX}=0.0$ \\
\hline A5 & $\mathrm{A}(1,2,7)$ & 120.173 & $-\mathrm{DE} / \mathrm{DX}=0.0$ \\
\hline A6 & $\mathrm{A}(3,2,7)$ & 120.4448 & $-\mathrm{DE} / \mathrm{DX}=0.0$ \\
\hline A7 & $\mathrm{A}(2,3,4)$ & 120.9566 & $-\mathrm{DE} / \mathrm{DX}=0.0$ \\
\hline A8 & $\mathrm{A}(2,3,8)$ & 119.6007 & $-\mathrm{DE} / \mathrm{DX}=0.0$ \\
\hline A9 & $\mathrm{A}(4,3,8)$ & 119.4401 & $-\mathrm{DE} / \mathrm{DX}=0.0$ \\
\hline A 10 & $\mathrm{~A}(3,4,5)$ & 118.7441 & $-\mathrm{DE} / \mathrm{DX}=0.0$ \\
\hline A11 & $\mathrm{A}(3,4,11)$ & 118.5323 & $-\mathrm{DE} / \mathrm{DX}=0.0$ \\
\hline A12 & $\mathrm{A}(5,4,11)$ & 122.6665 & $-\mathrm{DE} / \mathrm{DX}=0.0$ \\
\hline A13 & $\mathrm{A}(4,5,6)$ & 120.5644 & $-\mathrm{DE} / \mathrm{DX}=0.0$ \\
\hline A14 & $\mathrm{A}(4,5,9)$ & 120.1879 & $-\mathrm{DE} / \mathrm{DX}=0.0$ \\
\hline A15 & $\mathrm{A}(6,5,9)$ & 119.2311 & $-\mathrm{DE} / \mathrm{DX}=$ \\
\hline A16 & $\mathrm{A}(1,6,5)$ & 119.7175 & $-\mathrm{DE} / \mathrm{DX}=0.0$ \\
\hline A17 & $\mathrm{A}(1,6,10)$ & 120.0666 & $-\mathrm{DE} / \mathrm{DX}=0.0$ \\
\hline A18 & $\mathrm{A}(5,6,10)$ & 120.2001 & $-\mathrm{DE} / \mathrm{DX}=0.0$ \\
\hline A19 & $\mathrm{A}(4,11,16)$ & 131.9621 & $-\mathrm{DE} / \mathrm{DX}=0.0$ \\
\hline A 20 & $\mathrm{~A}(4,11,18)$ & 116.5506 & $-\mathrm{DE} / \mathrm{DX}=0.0$ \\
\hline A21 & $\mathrm{A}(16,11,18)$ & 111.0318 & $-\mathrm{DE} / \mathrm{DX}=0.0$ \\
\hline A22 & $\mathrm{A}(13,12,14)$ & 108.2527 & $-\mathrm{DE} / \mathrm{DX}=0.0$ \\
\hline A23 & $\mathrm{A}(13,12,15)$ & 110.15 & $-\mathrm{DE} / \mathrm{DX}=0.0$ \\
\hline A24 & $\mathrm{A}(13,12,16)$ & 113.2057 & $-\mathrm{DE} / \mathrm{DX}=$ \\
\hline A25 & $\mathrm{A}(14,12,15)$ & 107.7429 & $-\mathrm{DE} / \mathrm{DX}=0.0$ \\
\hline A26 & $\mathrm{A}(14,12,16)$ & 110.211 & $-\mathrm{DE} / \mathrm{DX}=0.0$ \\
\hline A27 & $\mathrm{A}(15,12,16)$ & 107.1395 & $-\mathrm{DE} / \mathrm{DX}=0.0$ \\
\hline A28 & $\mathrm{A}(11,16,12)$ & 118.8879 & $-\mathrm{DE} / \mathrm{DX}=0.0$ \\
\hline A 29 & $\mathrm{~A}(11,16,17)$ & 119.103 & $-\mathrm{DE} / \mathrm{DX}=0.0$ \\
\hline A 30 & $\mathrm{~A}(12,16,17)$ & 122.0047 & $-\mathrm{DE} / \mathrm{DX}=0.0$ \\
\hline D1 & $\mathrm{D}(6,1,2,3)$ & -1.0596 & $-\mathrm{DE} / \mathrm{DX}=0.0$ \\
\hline D2 & $\mathrm{D}(6,1,2,7)$ & 179.7271 & $-\mathrm{DE} / \mathrm{DX}=0.0$ \\
\hline D3 & $\mathrm{D}(19,1,2,3)$ & 179.3779 & $-\mathrm{DE} / \mathrm{DX}=0.0$ \\
\hline D4 & $\mathrm{D}(19,1,2,7)$ & 0.1646 & $-\mathrm{DE} / \mathrm{DX}=0.0$ \\
\hline D5 & $\mathrm{D}(2,1,6,5)$ & -0.1339 & $-\mathrm{DE} / \mathrm{DX}=0.0$ \\
\hline D6 & $\mathrm{D}(2,1,6,10)$ & -178.6956 & $-\mathrm{DE} / \mathrm{DX}=0.0$ \\
\hline D7 & $\mathrm{D}(19,1,6,5)$ & 179.4284 & $-\mathrm{DE} / \mathrm{DX}=0.0$ \\
\hline D8 & $\mathrm{D}(19,1,6,10)$ & 0.8668 & $-\mathrm{DE} / \mathrm{DX}=0.0$ \\
\hline D9 & $\mathrm{D}(1,2,3,4)$ & 0.7782 & $-\mathrm{DE} / \mathrm{DX}=0.0$ \\
\hline
\end{tabular}




\begin{tabular}{|c|c|c|c|}
\hline D10 & $\mathrm{D}(1,2,3,8)$ & -178.631 & $-\mathrm{DE} / \mathrm{DX}=$ \\
\hline D11 & $\mathrm{D}(7,2,3,4)$ & 179.9893 & $-\mathrm{DE} / \mathrm{DX}=0.0$ \\
\hline D12 & $\mathrm{D}(7,2,3,8)$ & 0.5801 & $-\mathrm{DE} / \mathrm{DX}=0.0$ \\
\hline D13 & $\mathrm{D}(2,3,4,5)$ & 0.6807 & $-\mathrm{DE} / \mathrm{DX}=0.0$ \\
\hline D14 & $\mathrm{D}(2,3,4,11)$ & 178.0073 & $-\mathrm{DE} / \mathrm{DX}=0.0$ \\
\hline D15 & $\mathrm{D}(8,3,4,5)$ & -179.9091 & $-\mathrm{DE} / \mathrm{DX}=0.0$ \\
\hline D16 & $\mathrm{D}(8,3,4,11)$ & -2.5825 & $-\mathrm{DE} / \mathrm{DX}=0.0$ \\
\hline D17 & $\mathrm{D}(3,4,5,6)$ & -1.889 & $-\mathrm{DE} / \mathrm{DX}=0.0$ \\
\hline D18 & $\mathrm{D}(3,4,5,9)$ & 176.6144 & $-\mathrm{DE} / \mathrm{DX}=0.0$ \\
\hline D19 & $\mathrm{D}(11,4,5,6)$ & -179.0989 & $-\mathrm{DE} / \mathrm{DX}=0.0$ \\
\hline D20 & $\mathrm{D}(11,4,5,9)$ & -0.5955 & $-\mathrm{DE} / \mathrm{DX}=0.0$ \\
\hline $\mathrm{D} 21$ & $\mathrm{D}(3,4,11,16)$ & 139.9681 & $-\mathrm{DE} / \mathrm{DX}=0.0$ \\
\hline D22 & $\mathrm{D}(3,4,11,18)$ & -31.4723 & $-\mathrm{DE} / \mathrm{DX}=0.0$ \\
\hline D23 & $\mathrm{D}(5,4,11,16)$ & -42.8164 & $-\mathrm{DE} / \mathrm{DX}=0.0$ \\
\hline D24 & $\mathrm{D}(5,4,11,18)$ & 145.7432 & $-\mathrm{DE} / \mathrm{DX}=0.0$ \\
\hline D25 & $\mathrm{D}(4,5,6,1)$ & 1.6283 & $-\mathrm{DE} / \mathrm{DX}=0.0$ \\
\hline D26 & $\mathrm{D}(4,5,6,10)$ & -179.8121 & $-\mathrm{DE} / \mathrm{DX}=0.0$ \\
\hline D27 & $\mathrm{D}(9,5,6,1)$ & -176.8893 & $-\mathrm{DE} / \mathrm{DX}=0.0$ \\
\hline D28 & $\mathrm{D}(9,5,6,10)$ & 1.6704 & $-\mathrm{DE} / \mathrm{DX}=0.0$ \\
\hline D29 & $\mathrm{D}(4,11,16,12)$ & 0.7638 & $-\mathrm{DE} / \mathrm{DX}=0.0$ \\
\hline D30 & $\mathrm{D}(4,11,16,17)$ & -178.4854 & $-\mathrm{DE} / \mathrm{DX}=0.0$ \\
\hline D31 & $\mathrm{D}(18,11,16,12)$ & 172.5628 & $-\mathrm{DE} / \mathrm{DX}=0.0$ \\
\hline D32 & $\mathrm{D}(18,11,16,17)$ & -6.6863 & $-\mathrm{DE} / \mathrm{DX}=0.0$ \\
\hline D33 & $\mathrm{D}(13,12,16,11)$ & -32.5332 & $-\mathrm{DE} / \mathrm{DX}=0.0$ \\
\hline D34 & $\mathrm{D}(13,12,16,17)$ & 146.6932 & $-\mathrm{DE} / \mathrm{DX}=0.0$ \\
\hline D35 & $\mathrm{D}(14,12,16,11)$ & 88.8641 & $-\mathrm{DE} / \mathrm{DX}=0.0$ \\
\hline D36 & $\mathrm{D}(14,12,16,17)$ & -91.9095 & $-\mathrm{DE} / \mathrm{DX}=0.0$ \\
\hline D37 & $\mathrm{D}(15,12,16,11)$ & -154.1759 & $-\mathrm{DE} / \mathrm{DX}=0.0$ \\
\hline D38 & $\mathrm{D}(15,12,16,17)$ & 25.0505 & $-\mathrm{DE} / \mathrm{DX}=0.0$ \\
\hline
\end{tabular}

\section{CARTESIAN COORDINATES}

\begin{tabular}{|c|c|c|c|c|c|}
\hline 1 & 6 & 0 & -.140508 & .077778 & .090286 \\
\hline 2 & 6 & 0 & -.050465 & .384642 & 1.447540 \\
\hline 3 & 6 & 0 & 1.178920 & .280328 & 2.090845 \\
\hline 4 & 6 & 0 & 2.329602 & -.112985 & 1.389775 \\
\hline 5 & 6 & 0 & 2.223158 & -.387107 & .018776 \\
\hline 6 & 6 & 0 & .989934 & -.308819 & -.626014 \\
\hline 7 & 1 & 0 & -.933960 & .689014 & 1.997139 \\
\hline 8 & 1 & 0 & 1.248787 & .496903 & 3.152959 \\
\hline 9 & 1 & 0 & 3.105627 & -.639099 & -.556343 \\
\hline 10 & 1 & 0 & .913886 & -.524969 & -1.685675 \\
\hline 11 & 7 & 0 & 3.557512 & -.160675 & 2.082751 \\
\hline 12 & 6 & 0 & 4.528871 & -2.243626 & 1.094879 \\
\hline 13 & 1 & 0 & 3.509857 & -2.608271 & .950098 \\
\hline 14 & 1 & 0 & 4.938845 & -1.973477 & .115948 \\
\hline 15 & 1 & 0 & 5.155053 & -3.033393 & 1.510916 \\
\hline 16 & 6 & 0 & 4.611799 & -1.062204 & 2.041663 \\
\hline 17 & 8 & 0 & 5.571157 & -.888539 & 2.777852 \\
\hline 18 & 1 & 0 & 3.679403 & .507235 & 2.834520 \\
\hline 19 & 17 & 0 & -1.692751 & .191011 & -.726425 \\
\hline
\end{tabular}

\section{S2.3. c}

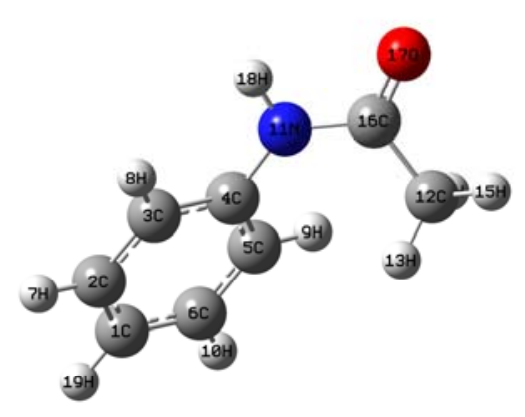

OPTIMIZED PARAMETERS

\begin{tabular}{|c|c|c|c|}
\hline R1 & $\mathrm{R}(1,2)$ & 1.3963 & $-\mathrm{DE} / \mathrm{DX}=0.0$ \\
\hline R2 & $\mathrm{R}(1,6)$ & 1.3948 & $-\mathrm{DE} / \mathrm{DX}=0.0$ \\
\hline R3 & $\mathrm{R}(1,19)$ & 1.0854 & $-\mathrm{DE} / \mathrm{DX}=0.0$ \\
\hline R4 & $\mathrm{R}(2,3)$ & 1.3924 & $-\mathrm{DE} / \mathrm{DX}=0.0$ \\
\hline R5 & $\mathrm{R}(2,7)$ & 1.086 & $-\mathrm{DE} / \mathrm{DX}=0.0$ \\
\hline R6 & $\mathrm{R}(3,4)$ & 1.4037 & $-\mathrm{DE} / \mathrm{DX}=0.0$ \\
\hline R7 & $\mathrm{R}(3,8)$ & 1.0866 & $-\mathrm{DE} / \mathrm{DX}=$ \\
\hline
\end{tabular}

\begin{tabular}{|c|c|c|c|}
\hline R8 & $\mathrm{R}(4,5)$ & 1.4022 & $-\mathrm{DE} / \mathrm{DX}=$ \\
\hline R9 & $\mathrm{R}(4,11)$ & 1.4132 & $-\mathrm{DE} / \mathrm{DX}=0.0$ \\
\hline R10 & $\mathrm{R}(5,6)$ & 1.3949 & $-\mathrm{DE} / \mathrm{DX}=0.0$ \\
\hline R11 & $\mathrm{R}(5,9)$ & 1.0834 & $-\mathrm{DE} / \mathrm{DX}=$ \\
\hline R12 & $\mathrm{R}(6,10)$ & 1.0861 & $-\mathrm{DE} / \mathrm{DX}=0.0$ \\
\hline R13 & $\mathrm{R}(11,16)$ & 1.3857 & $-\mathrm{DE} / \mathrm{DX}=0.0$ \\
\hline R14 & $\mathrm{R}(11,18)$ & 1.0129 & $-\mathrm{DE} / \mathrm{DX}=0.0$ \\
\hline R15 & $\mathrm{R}(12,13)$ & 1.0919 & $-\mathrm{DE} / \mathrm{DX}=0.0$ \\
\hline R16 & $\mathrm{R}(12,14)$ & 1.0951 & $-\mathrm{DE} / \mathrm{DX}=0.0$ \\
\hline R17 & $\mathrm{R}(12,15)$ & 1.0904 & $-\mathrm{DE} / \mathrm{DX}=0.0$ \\
\hline R18 & $\mathrm{R}(12,16)$ & 1.5167 & $-\mathrm{DE} / \mathrm{DX}=$ \\
\hline R19 & $\mathrm{R}(16,17)$ & 1.2227 & $-\mathrm{DE} / \mathrm{DX}=0.0$ \\
\hline A1 & $\mathrm{A}(2,1,6)$ & 119.291 & $-\mathrm{DE} / \mathrm{DX}=0.0$ \\
\hline A2 & $\mathrm{A}(2,1,19)$ & 120.3558 & $-\mathrm{DE} / \mathrm{DX}=0.0$ \\
\hline A3 & $\mathrm{A}(6,1,19)$ & 120.3511 & $-\mathrm{DE} / \mathrm{DX}=0.0$ \\
\hline A4 & $\mathrm{A}(1,2,3)$ & 120.3736 & $-\mathrm{DE} / \mathrm{DX}=0.0$ \\
\hline A5 & $\mathrm{A}(1,2,7)$ & 120.1831 & $-\mathrm{DE} / \mathrm{DX}=0.0$ \\
\hline A6 & $\mathrm{A}(3,2,7)$ & 119.4397 & $-\mathrm{DE} / \mathrm{DX}=0.0$ \\
\hline A7 & $\mathrm{A}(2,3,4)$ & 120.4703 & $-\mathrm{DE} / \mathrm{DX}=0.0$ \\
\hline A8 & $\mathrm{A}(2,3,8)$ & 120.2763 & $-\mathrm{DE} / \mathrm{DX}=$ \\
\hline A9 & $\mathrm{A}(4,3,8)$ & 119.2506 & $-\mathrm{DE} / \mathrm{DX}=0.0$ \\
\hline $\mathrm{A} 10$ & $\mathrm{~A}(3,4,5)$ & 119.0391 & $-\mathrm{DE} / \mathrm{DX}=0.0$ \\
\hline A11 & $\mathrm{A}(3,4,11)$ & 118.4097 & $-\mathrm{DE} / \mathrm{DX}=0.0$ \\
\hline A12 & $\mathrm{A}(5,4,11)$ & 122.497 & $-\mathrm{DE} / \mathrm{DX}=0.0$ \\
\hline A13 & $\mathrm{A}(4,5,6)$ & 120.0752 & $-\mathrm{DE} / \mathrm{DX}=0.0$ \\
\hline A14 & $\mathrm{A}(4,5,9)$ & 120.0268 & $-\mathrm{DE} / \mathrm{DX}=0.0$ \\
\hline A15 & $\mathrm{A}(6,5,9)$ & 119.8803 & $-\mathrm{DE} / \mathrm{DX}=0.0$ \\
\hline A16 & $\mathrm{A}(1,6,5)$ & 120.7196 & $-\mathrm{DE} / \mathrm{DX}=0.0$ \\
\hline A17 & $\mathrm{A}(1,6,10)$ & 120.071 & $-\mathrm{DE} / \mathrm{DX}=0.0$ \\
\hline A18 & $\mathrm{A}(5,6,10)$ & 119.1959 & $-\mathrm{DE} / \mathrm{DX}=0.0$ \\
\hline A19 & $\mathrm{A}(4,11,16)$ & 131.9322 & $-\mathrm{DE} / \mathrm{DX}=0.0$ \\
\hline A20 & $\mathrm{A}(4,11,18)$ & 116.5074 & $-\mathrm{DE} / \mathrm{DX}=0.0$ \\
\hline A21 & $\mathrm{A}(16,11,18)$ & 111.0621 & $-\mathrm{DE} / \mathrm{DX}=0.0$ \\
\hline A 22 & $\mathrm{~A}(13,12,14)$ & 108.1627 & $-\mathrm{DE} / \mathrm{DX}=0.0$ \\
\hline A 23 & $\mathrm{~A}(13,12,15)$ & 110.2424 & $-\mathrm{DE} / \mathrm{DX}=0.0$ \\
\hline $\mathrm{A} 24$ & $\mathrm{~A}(13,12,16)$ & 113.12 & $-\mathrm{DE} / \mathrm{DX}=0.0$ \\
\hline $\mathrm{A} 25$ & $\mathrm{~A}(14,12,15)$ & 107.8114 & $-\mathrm{DE} / \mathrm{DX}=0.0$ \\
\hline A26 & $\mathrm{A}(14,12,16)$ & 110.2338 & $-\mathrm{DE} / \mathrm{DX}=0.0$ \\
\hline A27 & $\mathrm{A}(15,12,16)$ & 107.1515 & $-\mathrm{DE} / \mathrm{DX}=0.0$ \\
\hline A 28 & $\mathrm{~A}(11,16,12)$ & 118.8431 & $-\mathrm{DE} / \mathrm{DX}=0.0$ \\
\hline A29 & $\mathrm{A}(11,16,17)$ & 119.2618 & $-\mathrm{DE} / \mathrm{DX}=0.0$ \\
\hline $\mathrm{A} 30$ & $\mathrm{~A}(12,16,17)$ & 121.8906 & $-\mathrm{DE} / \mathrm{DX}=0.0$ \\
\hline D1 & $\mathrm{D}(6,1,2,3)$ & -1.0534 & $-\mathrm{DE} / \mathrm{DX}=0.0$ \\
\hline D2 & $\mathrm{D}(6,1,2,7)$ & 179.6383 & $-\mathrm{DE} / \mathrm{DX}=0.0$ \\
\hline D3 & $\mathrm{D}(19,1,2,3)$ & 179.4734 & $-\mathrm{DE} / \mathrm{DX}=0.0$ \\
\hline D4 & $\mathrm{D}(19,1,2,7)$ & 0.1652 & $-\mathrm{DE} / \mathrm{DX}=0.0$ \\
\hline D5 & $\mathrm{D}(2,1,6,5)$ & -0.1389 & $-\mathrm{DE} / \mathrm{DX}=0.0$ \\
\hline D6 & $\mathrm{D}(2,1,6,10)$ & -178.7947 & $-\mathrm{DE} / \mathrm{DX}=0.0$ \\
\hline D7 & $\mathrm{D}(19,1,6,5)$ & 179.3343 & $-\mathrm{DE} / \mathrm{DX}=0.0$ \\
\hline D8 & $\mathrm{D}(19,1,6,10)$ & 0.6785 & $-\mathrm{DE} / \mathrm{DX}=0.0$ \\
\hline D9 & $\mathrm{D}(1,2,3,4)$ & 0.7506 & $-\mathrm{DE} / \mathrm{DX}=0.0$ \\
\hline D10 & $\mathrm{D}(1,2,3,8)$ & -178.6332 & $-\mathrm{DE} / \mathrm{DX}=0.0$ \\
\hline D11 & $\mathrm{D}(7,2,3,4)$ & -179.936 & $-\mathrm{DE} / \mathrm{DX}=0.0$ \\
\hline D12 & $\mathrm{D}(7,2,3,8)$ & 0.6802 & $-\mathrm{DE} / \mathrm{DX}=0.0$ \\
\hline D13 & $\mathrm{D}(2,3,4,5)$ & 0.7388 & $-\mathrm{DE} / \mathrm{DX}=0.0$ \\
\hline D14 & $\mathrm{D}(2,3,4,11)$ & 178.128 & $-\mathrm{DE} / \mathrm{DX}=0.0$ \\
\hline D15 & $\mathrm{D}(8,3,4,5)$ & -179.8711 & $-\mathrm{DE} / \mathrm{DX}=0.0$ \\
\hline D16 & $\mathrm{D}(8,3,4,11)$ & -2.482 & $-\mathrm{DE} / \mathrm{DX}=0.0$ \\
\hline D17 & $\mathrm{D}(3,4,5,6)$ & -1.9193 & $-\mathrm{DE} / \mathrm{DX}=0.0$ \\
\hline D18 & $\mathrm{D}(3,4,5,9)$ & 176.553 & $-\mathrm{DE} / \mathrm{DX}=0.0$ \\
\hline D19 & $\mathrm{D}(11,4,5,6)$ & -179.1965 & $-\mathrm{DE} / \mathrm{DX}=0.0$ \\
\hline D20 & $\mathrm{D}(11,4,5,9)$ & -0.7242 & $-\mathrm{DE} / \mathrm{DX}=0.0$ \\
\hline D21 & $\mathrm{D}(3,4,11,16)$ & 139.2692 & $-\mathrm{DE} / \mathrm{DX}=0.0$ \\
\hline D22 & $\mathrm{D}(3,4,11,18)$ & -31.7824 & $-\mathrm{DE} / \mathrm{DX}=0.0$ \\
\hline D23 & $\mathrm{D}(5,4,11,16)$ & -43.4372 & $-\mathrm{DE} / \mathrm{DX}=0.0$ \\
\hline D24 & $\mathrm{D}(5,4,11,18)$ & 145.5112 & $-\mathrm{DE} / \mathrm{DX}=0.0$ \\
\hline D25 & $\mathrm{D}(4,5,6,1)$ & 1.6363 & $-\mathrm{DE} / \mathrm{DX}=0.0$ \\
\hline D26 & $\mathrm{D}(4,5,6,10)$ & -179.6963 & $-\mathrm{DE} / \mathrm{DX}=0.0$ \\
\hline D27 & $\mathrm{D}(9,5,6,1)$ & -176.8383 & $-\mathrm{DE} / \mathrm{DX}=0.0$ \\
\hline D28 & $\mathrm{D}(9,5,6,10)$ & 1.8291 & $-\mathrm{DE} / \mathrm{DX}=0.0$ \\
\hline D29 & $\mathrm{D}(4,11,16,12)$ & 0.9689 & $-\mathrm{DE} / \mathrm{DX}=0.0$ \\
\hline D30 & $\mathrm{D}(4,11,16,17)$ & -178.27 & $-\mathrm{DE} / \mathrm{DX}=0.0$ \\
\hline D31 & $\mathrm{D}(18,11,16,12)$ & 172.3907 & $-\mathrm{DE} / \mathrm{DX}=0.0$ \\
\hline D32 & $\mathrm{D}(18,11,16,17)$ & -6.8481 & $-\mathrm{DE} / \mathrm{DX}=0.0$ \\
\hline D33 & $\mathrm{D}(13,12,16,11)$ & -32.7424 & $-\mathrm{DE} / \mathrm{DX}=0.0$ \\
\hline D34 & $\mathrm{D}(13,12,16,17)$ & 146.4755 & $-\mathrm{DE} / \mathrm{DX}=0.0$ \\
\hline D35 & $\mathrm{D}(14,12,16,11)$ & 88.4962 & $-\mathrm{DE} / \mathrm{DX}=0.0$ \\
\hline D36 & $\mathrm{D}(14,12,16,17)$ & -92.2859 & $-\mathrm{DE} / \mathrm{DX}=0.0$ \\
\hline D37 & $\mathrm{D}(15,12,16,11)$ & -154.4432 & $-\mathrm{DE} / \mathrm{DX}=0.0$ \\
\hline D38 & $\mathrm{D}(15,12,16,17)$ & 24.7747 & $-\mathrm{DE} / \mathrm{DX}=0.0$ \\
\hline
\end{tabular}

CARTESIAN COORDINATES

$\begin{array}{llllll}1 & 6 & 0 & -.153111 & .084826 & .091373 \\ 2 & 6 & 0 & -.043629 & .383969 & 1.450859 \\ 3 & 6 & 0 & 1.185310 & .281834 & 2.097498\end{array}$




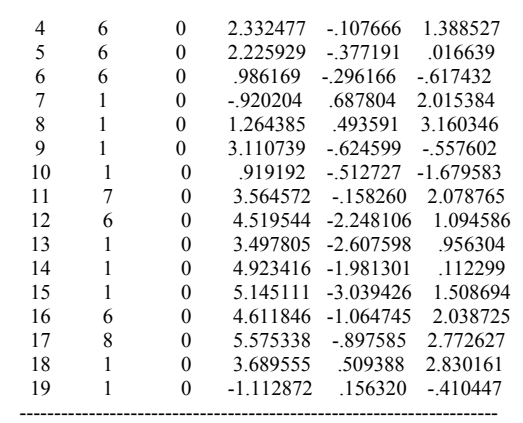

S2.4. d

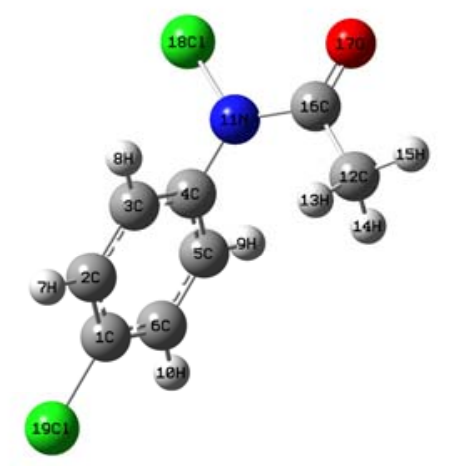

OPTIMIZED PARAMETERS

\begin{tabular}{|c|c|c|c|}
\hline R1 & $\mathrm{R}(1,2)$ & 1.3958 & $-\mathrm{DE} / \mathrm{DX}=0.0$ \\
\hline R2 & $\mathrm{R}(1,6)$ & 1.3956 & $-\mathrm{DE} / \mathrm{DX}=0.0$ \\
\hline R3 & $\mathrm{R}(1,19)$ & 1.7541 & $-\mathrm{DE} / \mathrm{DX}=0.0$ \\
\hline R4 & $\mathrm{R}(2,3)$ & 1.3928 & $-\mathrm{DE} / \mathrm{DX}=0.0$ \\
\hline R5 & $\mathrm{R}(2,7)$ & 1.084 & $-\mathrm{DE} / \mathrm{DX}=0.0$ \\
\hline R6 & $\mathrm{R}(3,4)$ & 1.399 & $-\mathrm{DE} / \mathrm{DX}=0.0$ \\
\hline R7 & $\mathrm{R}(3,8)$ & 1.085 & $-\mathrm{DE} / \mathrm{DX}=0.0$ \\
\hline R8 & $\mathrm{R}(4,5)$ & 1.3994 & $-\mathrm{DE} / \mathrm{DX}=0.0$ \\
\hline R9 & $\mathrm{R}(4,11)$ & 1.4276 & $-\mathrm{DE} / \mathrm{DX}=0.0$ \\
\hline R10 & $\mathrm{R}(5,6)$ & 1.393 & $-\mathrm{DE} / \mathrm{DX}=0.0$ \\
\hline R11 & $\mathrm{R}(5,9)$ & 1.0853 & $-\mathrm{DE} / \mathrm{DX}=0.0$ \\
\hline $\mathrm{R} 12$ & $\mathrm{R}(6,10)$ & 1.084 & $-\mathrm{DE} / \mathrm{DX}=0.0$ \\
\hline R13 & $\mathrm{R}(11,16)$ & 1.3959 & $-\mathrm{DE} / \mathrm{DX}=0.0$ \\
\hline R14 & $\mathrm{R}(11,18)$ & 1.7508 & $-\mathrm{DE} / \mathrm{DX}=0.0$ \\
\hline R15 & $\mathrm{R}(12,13)$ & 1.0924 & $-\mathrm{DE} / \mathrm{DX}=0.0$ \\
\hline R16 & $\mathrm{R}(12,14)$ & 1.0942 & $-\mathrm{DE} / \mathrm{DX}=0.0$ \\
\hline R17 & $\mathrm{R}(12,15)$ & 1.0897 & $-\mathrm{DE} / \mathrm{DX}=0.0$ \\
\hline R18 & $\mathrm{R}(12,16)$ & 1.5227 & $-\mathrm{DE} / \mathrm{DX}=0.0$ \\
\hline R19 & $\mathrm{R}(16,17)$ & 1.2132 & $-\mathrm{DE} / \mathrm{DX}=0.0$ \\
\hline A1 & $\mathrm{A}(2,1,6)$ & 121.3368 & $-\mathrm{DE} / \mathrm{DX}=0.0$ \\
\hline A2 & $\mathrm{A}(2,1,19)$ & 119.3329 & $-\mathrm{DE} / \mathrm{DX}=0.0$ \\
\hline A3 & $\mathrm{A}(6,1,19)$ & 119.3301 & $-\mathrm{DE} / \mathrm{DX}=0.0$ \\
\hline A4 & $\mathrm{A}(1,2,3)$ & 119.12 & $-\mathrm{DE} / \mathrm{DX}=0.0$ \\
\hline A5 & $\mathrm{A}(1,2,7)$ & 120.1005 & $-\mathrm{DE} / \mathrm{DX}=0.0$ \\
\hline A6 & $\mathrm{A}(3,2,7)$ & 120.7795 & $-\mathrm{DE} / \mathrm{DX}=0.0$ \\
\hline A7 & $\mathrm{A}(2,3,4)$ & 120.2349 & $-\mathrm{DE} / \mathrm{DX}=0.0$ \\
\hline A8 & $\mathrm{A}(2,3,8)$ & 120.4818 & $-\mathrm{DE} / \mathrm{DX}=0.0$ \\
\hline A9 & $\mathrm{A}(4,3,8)$ & 119.2832 & $-\mathrm{DE} / \mathrm{DX}=0.0$ \\
\hline A10 & $\mathrm{A}(3,4,5)$ & 119.9589 & $-\mathrm{DE} / \mathrm{DX}=0.0$ \\
\hline A11 & $\mathrm{A}(3,4,11)$ & 119.6187 & $-\mathrm{DE} / \mathrm{DX}=0.0$ \\
\hline A12 & $\mathrm{A}(5,4,11)$ & 120.419 & $-\mathrm{DE} / \mathrm{DX}=0.0$ \\
\hline A13 & $\mathrm{A}(4,5,6)$ & 120.2267 & $-\mathrm{DE} / \mathrm{DX}=0.0$ \\
\hline A14 & $\mathrm{A}(4,5,9)$ & 119.4853 & $-\mathrm{DE} / \mathrm{DX}=0.0$ \\
\hline A15 & $\mathrm{A}(6,5,9)$ & 120.2876 & $-\mathrm{DE} / \mathrm{DX}=0.0$ \\
\hline A16 & $\mathrm{A}(1,6,5)$ & 119.1126 & $-\mathrm{DE} / \mathrm{DX}=0.0$ \\
\hline A17 & $\mathrm{A}(1,6,10)$ & 120.1115 & $-\mathrm{DE} / \mathrm{DX}=0.0$ \\
\hline A18 & $\mathrm{A}(5,6,10)$ & 120.7749 & $-\mathrm{DE} / \mathrm{DX}=0.0$ \\
\hline A19 & $\mathrm{A}(4,11,16)$ & 127.1389 & $-\mathrm{DE} / \mathrm{DX}=0.0$ \\
\hline A 20 & $\mathrm{~A}(4,11,18)$ & 115.5633 & $-\mathrm{DE} / \mathrm{DX}=0.0$ \\
\hline
\end{tabular}

\begin{tabular}{|c|c|c|c|}
\hline $\mathrm{A} 21$ & $\mathrm{~A}(16,11,18)$ & 116.2788 & $-\mathrm{DE} / \mathrm{DX}=$ \\
\hline $\mathrm{A} 22$ & $\mathrm{~A}(13,12,14)$ & 107.7018 & $-\mathrm{DE} / \mathrm{DX}=0.0$ \\
\hline A 23 & $\mathrm{~A}(13,12,15)$ & 110.0237 & $-\mathrm{DE} / \mathrm{DX}=$ \\
\hline $\mathrm{A} 24$ & $\mathrm{~A}(13,12,16)$ & 112.3299 & $-\mathrm{DE} / \mathrm{DX}=$ \\
\hline $\mathrm{A} 25$ & $\mathrm{~A}(14,12,15)$ & 109.0206 & $-\mathrm{DE} / \mathrm{DX}=0.0$ \\
\hline A26 & $\mathrm{A}(14,12,16)$ & 110.6872 & $-\mathrm{DE} / \mathrm{DX}=0.0$ \\
\hline $\mathrm{A} 27$ & $\mathrm{~A}(15,12,16)$ & 107.0493 & $-\mathrm{DE} / \mathrm{DX}=$ \\
\hline $\mathrm{A} 28$ & $\mathrm{~A}(11,16,12)$ & 114.2459 & $-\mathrm{DE} / \mathrm{DX}=0.0$ \\
\hline A 29 & $\mathrm{~A}(11,16,17)$ & 122.531 & $-\mathrm{DE} / \mathrm{DX}=0.0$ \\
\hline $\mathrm{A} 30$ & $\mathrm{~A}(12,16,17)$ & 123.215 & $-\mathrm{DE} / \mathrm{DX}=0.0$ \\
\hline D1 & $\mathrm{D}(6,1,2,3)$ & -0.2955 & $-\mathrm{DE} / \mathrm{DX}=0.0$ \\
\hline D2 & $\mathrm{D}(6,1,2,7)$ & 179.77 & $-\mathrm{DE} / \mathrm{DX}=0.0$ \\
\hline D3 & $\mathrm{D}(19,1,2,3)$ & 179.8486 & $-\mathrm{DE} / \mathrm{DX}=0.0$ \\
\hline D4 & $\mathrm{D}(19,1,2,7)$ & -0.0859 & $-\mathrm{DE} / \mathrm{DX}=0.0$ \\
\hline D5 & $\mathrm{D}(2,1,6,5)$ & 0.0507 & $-\mathrm{DE} / \mathrm{DX}=0.0$ \\
\hline D6 & $\mathrm{D}(2,1,6,10)$ & -179.5811 & $-\mathrm{DE} / \mathrm{DX}=0.0$ \\
\hline D7 & $\mathrm{D}(19,1,6,5)$ & 179.9066 & $-\mathrm{DE} / \mathrm{DX}=0.0$ \\
\hline D8 & $\mathrm{D}(19,1,6,10)$ & 0.2748 & $-\mathrm{DE} / \mathrm{DX}=0.0$ \\
\hline D9 & $\mathrm{D}(1,2,3,4)$ & -0.2204 & $-\mathrm{DE} / \mathrm{DX}=0.0$ \\
\hline D10 & $\mathrm{D}(1,2,3,8)$ & 179.8228 & $-\mathrm{DE} / \mathrm{DX}=0.0$ \\
\hline D11 & $\mathrm{D}(7,2,3,4)$ & 179.7137 & $-\mathrm{DE} / \mathrm{DX}=0.0$ \\
\hline D12 & $\mathrm{D}(7,2,3,8)$ & -0.2431 & $-\mathrm{DE} / \mathrm{DX}=0.0$ \\
\hline D13 & $\mathrm{D}(2,3,4,5)$ & 0.9754 & $-\mathrm{DE} / \mathrm{DX}=0.0$ \\
\hline D14 & $\mathrm{D}(2,3,4,11)$ & -179.6948 & $-\mathrm{DE} / \mathrm{DX}=0.0$ \\
\hline D15 & $\mathrm{D}(8,3,4,5)$ & -179.0673 & $-\mathrm{DE} / \mathrm{DX}=0.0$ \\
\hline D16 & $\mathrm{D}(8,3,4,11)$ & 0.2626 & $-\mathrm{DE} / \mathrm{DX}=0.0$ \\
\hline D17 & $\mathrm{D}(3,4,5,6)$ & -1.2239 & $-\mathrm{DE} / \mathrm{DX}=0.0$ \\
\hline D18 & $\mathrm{D}(3,4,5,9)$ & 178.5442 & $-\mathrm{DE} / \mathrm{DX}=0.0$ \\
\hline D19 & $\mathrm{D}(11,4,5,6)$ & 179.4517 & $-\mathrm{DE} / \mathrm{DX}=0.0$ \\
\hline D20 & $\mathrm{D}(11,4,5,9)$ & -0.7802 & $-\mathrm{DE} / \mathrm{DX}=0.0$ \\
\hline D21 & $\mathrm{D}(3,4,11,16)$ & 104.0219 & $-\mathrm{DE} / \mathrm{DX}=0.0$ \\
\hline D22 & $\mathrm{D}(3,4,11,18)$ & -88.0395 & $-\mathrm{DE} / \mathrm{DX}=0.0$ \\
\hline D23 & $\mathrm{D}(5,4,11,16)$ & -76.6514 & $-\mathrm{DE} / \mathrm{DX}=0.0$ \\
\hline D24 & $\mathrm{D}(5,4,11,18)$ & 91.2872 & $-\mathrm{DE} / \mathrm{DX}=0.0$ \\
\hline D25 & $\mathrm{D}(4,5,6,1)$ & 0.7103 & $-\mathrm{DE} / \mathrm{DX}=0.0$ \\
\hline D26 & $\mathrm{D}(4,5,6,10)$ & -179.6604 & $-\mathrm{DE} / \mathrm{DX}=0.0$ \\
\hline D27 & $\mathrm{D}(9,5,6,1)$ & -179.0559 & $-\mathrm{DE} / \mathrm{DX}=0.0$ \\
\hline D28 & $\mathrm{D}(9,5,6,10)$ & 0.5734 & $-\mathrm{DE} / \mathrm{DX}=0.0$ \\
\hline D29 & $\mathrm{D}(4,11,16,12)$ & -10.3462 & $-\mathrm{DE} / \mathrm{DX}=0.0$ \\
\hline D30 & $\mathrm{D}(4,11,16,17)$ & 170.6554 & $-\mathrm{DE} / \mathrm{DX}=0.0$ \\
\hline D31 & $\mathrm{D}(18,11,16,12)$ & -178.2102 & $-\mathrm{DE} / \mathrm{DX}=0.0$ \\
\hline D32 & $\mathrm{D}(18,11,16,17)$ & 2.7913 & $-\mathrm{DE} / \mathrm{DX}=0.0$ \\
\hline D33 & $\mathrm{D}(13,12,16,11)$ & -46.4308 & $-\mathrm{DE} / \mathrm{DX}=0.0$ \\
\hline D34 & $\mathrm{D}(13,12,16,17)$ & 132.5599 & $-\mathrm{DE} / \mathrm{DX}=0.0$ \\
\hline D35 & $\mathrm{D}(14,12,16,11)$ & 73.9982 & $-\mathrm{DE} / \mathrm{DX}=0.0$ \\
\hline D36 & $\mathrm{D}(14,12,16,17)$ & -107.0111 & $-\mathrm{DE} / \mathrm{DX}=0.0$ \\
\hline D37 & $\mathrm{D}(15,12,16,11)$ & -167.3043 & $-\mathrm{DE} / \mathrm{DX}=0.0$ \\
\hline D38 & $\mathrm{D}(15,12,16,17)$ & 11.6864 & $-\mathrm{DE} / \mathrm{DX}=0.0$ \\
\hline
\end{tabular}

CARTESIAN COORDINATES

$\begin{array}{cccccc}1 & 6 & 0 & -.037088 & -.014145 & .046406 \\ 2 & 6 & 0 & .043655 & -.093219 & 1.437604 \\ 3 & 6 & 0 & 1.296429 & -.075253 & 2.045929 \\ 4 & 6 & 0 & 2.455899 & .016267 & 1.268374 \\ 5 & 6 & 0 & 2.357675 & .110694 & -.124419 \\ 6 & 6 & 0 & 1.109552 & .088322 & -.742512 \\ 7 & 1 & 0 & -.860199 & -.168527 & 2.031202 \\ 8 & 1 & 0 & 1.384771 & -.132624 & 3.125781 \\ 9 & 1 & 0 & 3.261007 & .205243 & -.718432 \\ 10 & 1 & 0 & 1.023429 & .155359 & -1.820986 \\ 11 & 7 & 0 & 3.733469 & .027039 & 1.905256 \\ 12 & 6 & 0 & 4.099038 & -2.364797 & 1.507148 \\ 13 & 1 & 0 & 3.113262 & -2.578366 & 1.926544 \\ 14 & 1 & 0 & 4.008417 & -2.385748 & .416906 \\ 15 & 1 & 0 & 4.810721 & -3.128729 & 1.819098 \\ 16 & 6 & 0 & 4.649009 & -1.024343 & 1.975555 \\ 17 & 8 & 0 & 5.778733 & -.878870 & 2.393302 \\ 18 & 17 & 0 & 4.324967 & 1.603404 & 2.385508 \\ 19 & 17 & 0 & -1.611823 & -.040331 & -.725812 \\ ------------------------------------------------\end{array}$

S2.4. e 


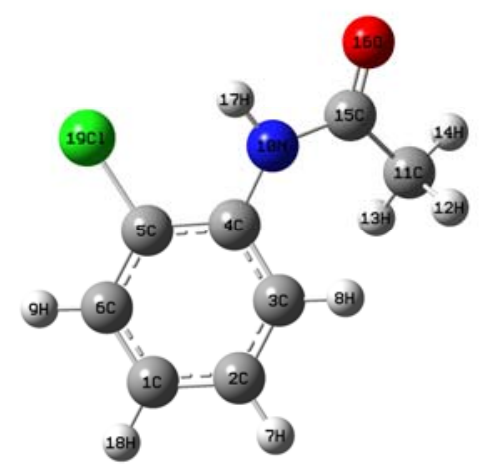

OPTIMIZED PARAMETERS

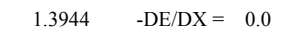

$\begin{array}{llll}\mathrm{R} 3 & \mathrm{R}(1,18) & 1.085 & -\mathrm{DE} / \mathrm{DX}=0.0\end{array}$

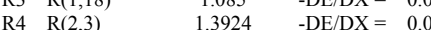

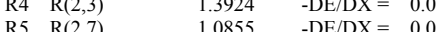

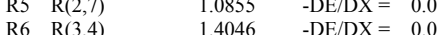

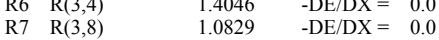

$\begin{array}{llll}\mathrm{R} 8 & \mathrm{R}(4,5) & 1.4095 & -\mathrm{DE} / \mathrm{DX}=0.0\end{array}$

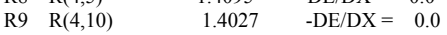

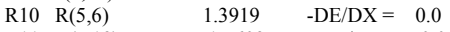

$\begin{array}{llll}\mathrm{R} 11 & \mathrm{R}(5,19) & 1.7609 & -\mathrm{DE} / \mathrm{DX}=0.0 \\ \mathrm{R} 12 & \mathrm{R}(6,9) & 1.0842 & -\mathrm{DE} / \mathrm{DX}=0.0\end{array}$

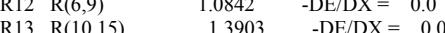

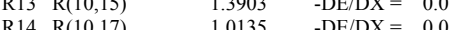

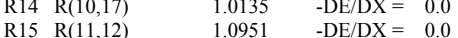

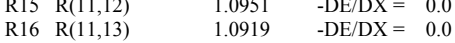

R17 R(11,14) $1.0904 \quad$-DE/DX $=0.0$

$\mathrm{R} 18 \mathrm{R}(11,15) \quad 1.5162 \quad-\mathrm{DE} / \mathrm{DX}=0.0$

R19 R $(15,16) \quad 1.2207 \quad-\mathrm{DE} / \mathrm{DX}=0.0$

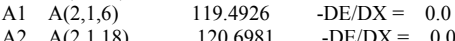

A3 $\mathrm{A}(6,1,18) \quad 119.8061 \quad-\mathrm{DE} / \mathrm{DX}=0.0$

A4 $\mathrm{A}(1,2,3) \quad 120.4411 \quad-\mathrm{DE} / \mathrm{DX}=0.0$

$\mathrm{A} 5 \mathrm{~A}(1,2,7) \quad 120.2493 \quad \mathrm{DE} / \mathrm{DX}=0.0$

A $A(1,2,7) \quad 120.2493 \quad-D E / D X=0.0$

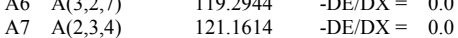

A8 $\quad \mathrm{A}(2,3,8) \quad 119.7903 \quad-\mathrm{DE} / \mathrm{DX}=0.0$

A9 $\mathrm{A}(4,3,8) \quad 119.032 \quad-\mathrm{DE} / \mathrm{DX}=0.0$

$\begin{array}{llll}\mathrm{A} 10 & \mathrm{~A}(3,4,5) & 117.3281 & -\mathrm{DE} / \mathrm{DX}=0.0\end{array}$

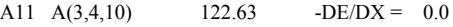

A12 $\mathrm{A}(5,4,10) \quad 119.9793 \quad-\mathrm{DE} / \mathrm{DX}=0.0$

$\begin{array}{llll}\mathrm{A} 13 & \mathrm{~A}(4,5,6) & 121.6803 & -\mathrm{DE} / \mathrm{DX}=0.0\end{array}$

$\mathrm{A} 14 \mathrm{~A}(4,5,19) \quad 119.6392 \quad-\mathrm{DE} / \mathrm{DX}=0.0$

A14 A $(4,5,19) \quad 119.6392 \quad-D E / D X=0.0$

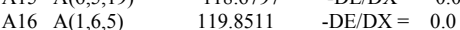

$\mathrm{A} 17 \mathrm{~A}(1,6,9) \quad 120.9811 \quad-\mathrm{DE} / \mathrm{DX}=0.0$

$\mathrm{A} 18 \mathrm{~A}(5,6,9) \quad 119.1657 \quad-\mathrm{DE} / \mathrm{DX}=0.0$

A19 $\mathrm{A}(4,10,15) \quad 131.7903 \quad-\mathrm{DE} / \mathrm{DX}=0.0$

A20 $\mathrm{A}(4,10,17) \quad 116.3315 \quad-\mathrm{DE} / \mathrm{DX}=0.0$

A21 $\mathrm{A}(15,10,17) \quad 111.1777 \quad-\mathrm{DE} / \mathrm{DX}=0.0$

A22 $\mathrm{A}(12,11,13) \quad 108.2885 \quad-\mathrm{DE} / \mathrm{DX}=0.0$

$107.7244-\mathrm{DE} / \mathrm{DX}=0.0$

A23 A (12,11,14) $\quad 107.7244 \quad-\mathrm{DE} / \mathrm{DX}=0.0$

$\mathrm{A} 25 \mathrm{~A}(13,11,14)-110.1523 \quad-\mathrm{DE} / \mathrm{DX}=0.0$

A25 A (13,11,14)

A26 $\mathrm{A}(13,11,15) \quad 113.2109 \quad-\mathrm{DE} / \mathrm{DX}=0.0$

A27 $\mathrm{A}(14,11,15) \quad 107.0101 \quad-\mathrm{DE} / \mathrm{DX}=0.0$

A28 $\mathrm{A}(10,15,11) \quad 119.1926 \quad-\mathrm{DE} / \mathrm{DX}=0.0$

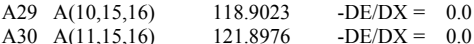

A30 A $(11,15,16)$

D1 $\mathrm{D}(6,1,2,3)$

D2 $\mathrm{D}(6,1,2,7)$

D3 $\mathrm{D}(18,1,2,3)$

D4 $\mathrm{D}(18,1,2,7)$

D5 $\mathrm{D}(2,1,6,5)$

D6 $\mathrm{D}(2,1,6,9)$

D7 $\mathrm{D}(18,1,6,5)$

D8 $\quad \mathrm{D}(18,1,6,9)$

D9 $\mathrm{D}(1,2,3,4)$

D10 $\mathrm{D}(1,2,3,8)$

D11 $\mathrm{D}(7,2,3,4)$

D12 $\mathrm{D}(7,2,3,8)$

$\begin{array}{ll}\text { D12 } & \mathrm{D}(7,2,3,8) \\ \mathrm{D}(2,3,4,5)\end{array}$

D14 D $(2,3,4,10)$

$\mathrm{D} 15 \mathrm{D}(8,3,4,5)$
$-0.032$

$178.537 \quad-\mathrm{DE} / \mathrm{DX}=0.0$

$-179.3832-\mathrm{DE} / \mathrm{DX}=0.0$

$0.8141 \quad-\mathrm{DE} / \mathrm{DX}=0.0$

$1.191-\mathrm{DE} / \mathrm{DX}=0.0$

$-179.3427 \quad-\mathrm{DE} / \mathrm{DX}=0.0$

$-179.452 \quad-\mathrm{DE} / \mathrm{DX}=0.0$

$0.0143 \quad-\mathrm{DE} / \mathrm{DX}=0.0$

$-1.8437 \quad-\mathrm{DE} / \mathrm{DX}=0.0$

$176.6745 \quad-\mathrm{DE} / \mathrm{DX}=0.0$

$179.5737-\mathrm{DE} / \mathrm{DX}=0.0$

$-1.9081 \quad-\mathrm{DE} / \mathrm{DX}=0.0$

$179.5712-\mathrm{DE} / \mathrm{DX}=0.0$

$\begin{array}{rrr}179.5712 & -\mathrm{DE} / \mathrm{DX}= & 0.0 \\ -176.0762 & -\mathrm{DE} / \mathrm{DX}= & 0.0\end{array}$

\begin{tabular}{|c|c|c|c|}
\hline D16 & $\mathrm{D}(8,3,4,10)$ & 1.0419 & -DE/DX = \\
\hline D17 & $\mathrm{D}(3,4,5,6)$ & -1.2827 & $-\mathrm{DE} / \mathrm{DX}=0.0$ \\
\hline D18 & $\mathrm{D}(3,4,5,19)$ & 178.3848 & $-\mathrm{DE} / \mathrm{DX}=0.0$ \\
\hline D19 & $\mathrm{D}(10,4,5,6)$ & -178.4808 & $-\mathrm{DE} / \mathrm{DX}=0.0$ \\
\hline D20 & $\mathrm{D}(10,4,5,19)$ & 1.1866 & $-\mathrm{DE} / \mathrm{DX}=0.0$ \\
\hline D21 & $\mathrm{D}(3,4,10,15)$ & 41.0256 & $-\mathrm{DE} / \mathrm{DX}=0.0$ \\
\hline D22 & $\mathrm{D}(3,4,10,17)$ & -149.5601 & $-\mathrm{DE} / \mathrm{DX}=0.0$ \\
\hline D23 & $\mathrm{D}(5,4,10,15)$ & -141.9302 & $-\mathrm{DE} / \mathrm{DX}=0.0$ \\
\hline D24 & $\mathrm{D}(5,4,10,17)$ & 27.4841 & $-\mathrm{DE} / \mathrm{DX}=0.0$ \\
\hline D25 & $\mathrm{D}(4,5,6,1)$ & -0.5167 & $-\mathrm{DE} / \mathrm{DX}=0.0$ \\
\hline D26 & $\mathrm{D}(4,5,6,9)$ & -179.9926 & $-\mathrm{DE} / \mathrm{DX}=0.0$ \\
\hline D27 & $\mathrm{D}(19,5,6,1)$ & 179.8128 & $-\mathrm{DE} / \mathrm{DX}=0.0$ \\
\hline D28 & $\mathrm{D}(19,5,6,9)$ & 0.3369 & $-\mathrm{DE} / \mathrm{DX}=0.0$ \\
\hline D29 & $\mathrm{D}(4,10,15,11)$ & 0.2187 & $-\mathrm{DE} / \mathrm{DX}=0.0$ \\
\hline D30 & $\mathrm{D}(4,10,15,16)$ & 179.2449 & $-\mathrm{DE} / \mathrm{DX}=0.0$ \\
\hline D31 & $\mathrm{D}(17,10,15,11)$ & -169.6113 & $-\mathrm{DE} / \mathrm{DX}=0.0$ \\
\hline D32 & $\mathrm{D}(17,10,15,16)$ & 9.4149 & $-\mathrm{DE} / \mathrm{DX}=0.0$ \\
\hline D33 & $\mathrm{D}(12,11,15,10)$ & -88.9749 & $-\mathrm{DE} / \mathrm{DX}=0.0$ \\
\hline D34 & $\mathrm{D}(12,11,15,16)$ & 92.0292 & $-\mathrm{DE} / \mathrm{DX}=0.0$ \\
\hline D35 & $\mathrm{D}(13,11,15,10)$ & 32.5543 & $-\mathrm{DE} / \mathrm{DX}=0.0$ \\
\hline D36 & $\mathrm{D}(13,11,15,16)$ & -146.4416 & $-\mathrm{DE} / \mathrm{DX}=0.0$ \\
\hline D37 & $\mathrm{D}(14,11,15,10)$ & 154.101 & $-\mathrm{DE} / \mathrm{DX}=0.0$ \\
\hline D38 & $\mathrm{D}(14,11,15,16)$ & -24.8948 & $-\mathrm{DE} / \mathrm{DX}=0.0$ \\
\hline
\end{tabular}

CARTESIAN COORDINATES

$\begin{array}{cccccc}1 & 6 & 0 & -.085615 & .097436 & -.037192 \\ 2 & 6 & 0 & .193730 & -.529992 & 1.176360 \\ 3 & 6 & 0 & 1.500253 & -.593115 & 1.653573 \\ 4 & 6 & 0 & 2.570696 & -.064650 & .913440 \\ 5 & 6 & 0 & 2.261946 & .577027 & -.302930 \\ 6 & 6 & 0 & .954494 & .663824 & -.772298 \\ 7 & 1 & 0 & -.609700 & -.954293 & 1.770242 \\ 8 & 1 & 0 & 1.698483 & -1.034857 & 2.622175 \\ 9 & 1 & 0 & .763313 & 1.166730 & -1.713628 \\ 10 & 7 & 0 & 3.896964 & -.103610 & 1.368417 \\ 11 & 6 & 0 & 3.955929 & -2.418116 & 2.331503 \\ 12 & 1 & 0 & 3.468596 & -2.373835 & 3.311225 \\ 13 & 1 & 0 & 3.209187 & -2.710027 & 1.590241 \\ 14 & 1 & 0 & 4.744280 & -3.169391 & 2.386543 \\ 15 & 6 & 0 & 4.625508 & -1.094834 & 2.016110 \\ 16 & 8 & 0 & 5.793453 & -.879439 & 2.298438 \\ 17 & 1 & 0 & 4.497928 & .655046 & 1.067548 \\ 18 & 1 & 0 & -1.103416 & .157757 & -.408173 \\ 19 & 17 & 0 & 3.552616 & 1.298998 & -1.258732\end{array}$

S2.5. f

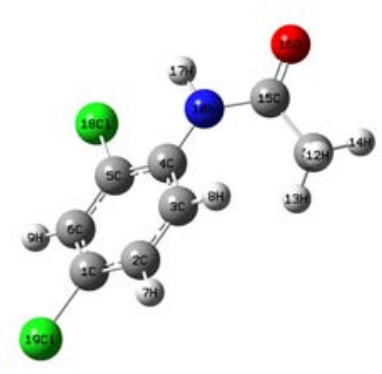

OPTIMIZED PARAMETERS

\begin{tabular}{|c|c|c|c|}
\hline R1 & $\mathrm{R}(1,2)$ & 1.3929 & $-\mathrm{DE} / \mathrm{DX}=0.0$ \\
\hline R2 & $\mathrm{R}(1,6)$ & 1.3927 & $-\mathrm{DE} / \mathrm{DX}=$ \\
\hline R3 & $\mathrm{R}(1,19)$ & 1.7542 & $-\mathrm{DE} / \mathrm{DX}=0.0$ \\
\hline R4 & $\mathrm{R}(2,3)$ & 1.3915 & $-\mathrm{DE} / \mathrm{DX}=0.0$ \\
\hline R5 & $\mathrm{R}(2,7)$ & 1.0837 & $-\mathrm{DE} / \mathrm{DX}=0.0$ \\
\hline R6 & $\mathrm{R}(3,4)$ & 1.4042 & $-\mathrm{DE} / \mathrm{DX}=0.0$ \\
\hline R7 & $\mathrm{R}(3,8)$ & 1.0826 & $-\mathrm{DE} / \mathrm{DX}=$ \\
\hline R8 & $\mathrm{R}(4,5)$ & 1.4098 & $-\mathrm{DE} / \mathrm{DX}=$ \\
\hline R9 & $\mathrm{R}(4,10)$ & 1.401 & $-\mathrm{DE} / \mathrm{DX}=0.0$ \\
\hline R10 & $\mathrm{R}(5,6)$ & 1.3917 & $-\mathrm{DE} / \mathrm{DX}=0.0$ \\
\hline R11 & $\mathrm{R}(5,18)$ & 1.757 & $-\mathrm{DE} / \mathrm{DX}=$ \\
\hline R12 & $\mathrm{R}(6,9)$ & 1.0828 & $-\mathrm{DE} / \mathrm{DX}=0.0$ \\
\hline R13 & $\mathrm{R}(10,15)$ & 1.3921 & $-\mathrm{DE} / \mathrm{DX}=0.0$ \\
\hline R14 & $\mathrm{R}(10,17)$ & 1.0136 & $-\mathrm{DE} / \mathrm{DX}=$ \\
\hline R15 & $\mathrm{R}(11,12)$ & 1.0952 & $-\mathrm{DE} / \mathrm{DX}=0.0$ \\
\hline
\end{tabular}




\begin{tabular}{|c|c|c|c|}
\hline R16 & $\mathrm{R}(11,13)$ & 1.092 & $-\mathrm{DE} / \mathrm{DX}=0.0$ \\
\hline R17 & $\mathrm{R}(11,14)$ & 1.0903 & $-\mathrm{DE} / \mathrm{DX}=0.0$ \\
\hline R18 & $\mathrm{R}(11,15)$ & 1.5158 & $-\mathrm{DE} / \mathrm{DX}=0.0$ \\
\hline R19 & $\mathrm{R}(15,16)$ & 1.2199 & $-\mathrm{DE} / \mathrm{DX}=$ \\
\hline A1 & $\mathrm{A}(2,1,6)$ & 120.6788 & $-\mathrm{DE} / \mathrm{DX}=0.0$ \\
\hline A2 & $\mathrm{A}(2,1,19)$ & 119.9625 & $-\mathrm{DE} / \mathrm{DX}=0.0$ \\
\hline $\mathrm{A} 3$ & $\mathrm{~A}(6,1,19)$ & 119.3562 & $-\mathrm{DE} / \mathrm{DX}=$ \\
\hline A4 & $\mathrm{A}(1,2,3)$ & 119.4566 & $-\mathrm{DE} / \mathrm{DX}=0.0$ \\
\hline A5 & $\mathrm{A}(1,2,7)$ & 120.2429 & $-\mathrm{DE} / \mathrm{DX}=0.0$ \\
\hline A6 & $\mathrm{A}(3,2,7)$ & 120.283 & $-\mathrm{DE} / \mathrm{DX}=0.0$ \\
\hline A7 & $\mathrm{A}(2,3,4)$ & 121.6813 & $-\mathrm{DE} / \mathrm{DX}=0.0$ \\
\hline A8 & $\mathrm{A}(2,3,8)$ & 119.1188 & $-\mathrm{DE} / \mathrm{DX}=0.0$ \\
\hline A9 & $\mathrm{A}(4,3,8)$ & 119.1844 & $-\mathrm{DE} / \mathrm{DX}=0.0$ \\
\hline A 10 & $\mathrm{~A}(3,4,5)$ & 117.1298 & $-\mathrm{DE} / \mathrm{DX}=0.0$ \\
\hline A11 & $\mathrm{A}(3,4,10)$ & 122.7373 & $-\mathrm{DE} / \mathrm{DX}=0.0$ \\
\hline A12 & $\mathrm{A}(5,4,10)$ & 120.0682 & $-\mathrm{DE} / \mathrm{DX}=$ \\
\hline A13 & $\mathrm{A}(4,5,6)$ & 121.9629 & $-\mathrm{DE} / \mathrm{DX}=0.0$ \\
\hline A14 & $\mathrm{A}(4,5,18)$ & 119.7589 & $-\mathrm{DE} / \mathrm{DX}=0.0$ \\
\hline A15 & $\mathrm{A}(6,5,18)$ & 118.2774 & $-\mathrm{DE} / \mathrm{DX}=0.0$ \\
\hline A16 & $\mathrm{A}(1,6,5)$ & 119.0439 & $-\mathrm{DE} / \mathrm{DX}=0.0$ \\
\hline A17 & $\mathrm{A}(1,6,9)$ & 120.8739 & $-\mathrm{DE} / \mathrm{DX}=0.0$ \\
\hline A18 & $\mathrm{A}(5,6,9)$ & 120.0792 & $-\mathrm{DE} / \mathrm{DX}=0.0$ \\
\hline A19 & $\mathrm{A}(4,10,15)$ & 131.753 & $-\mathrm{DE} / \mathrm{DX}=0.0$ \\
\hline A 20 & $\mathrm{~A}(4,10,17)$ & 116.4001 & $-\mathrm{DE} / \mathrm{DX}=0.0$ \\
\hline A21 & $\mathrm{A}(15,10,17)$ & 111.1631 & $-\mathrm{DE} / \mathrm{DX}=0.0$ \\
\hline A22 & $\mathrm{A}(12,11,13)$ & 108.3503 & $-\mathrm{DE} / \mathrm{DX}=$ \\
\hline A23 & $\mathrm{A}(12,11,14)$ & 107.6917 & $-\mathrm{DE} / \mathrm{DX}=$ \\
\hline A24 & $\mathrm{A}(12,11,15)$ & 110.3216 & $-\mathrm{DE} / \mathrm{DX}=$ \\
\hline A25 & $\mathrm{A}(13,11,14)$ & 110.0842 & $-\mathrm{DE} / \mathrm{DX}=$ \\
\hline A26 & $\mathrm{A}(13,11,15)$ & 113.2386 & $-\mathrm{DE} / \mathrm{DX}=$ \\
\hline A27 & $\mathrm{A}(14,11,15)$ & 107.0183 & $-\mathrm{DE} / \mathrm{DX}=$ \\
\hline A28 & $\mathrm{A}(10,15,11)$ & 119.1792 & $-\mathrm{DE} / \mathrm{DX}=$ \\
\hline A29 & $\mathrm{A}(10,15,16)$ & 118.7854 & $-\mathrm{DE} / \mathrm{DX}=$ \\
\hline A30 & $\mathrm{A}(11,15,16)$ & 122.0286 & $-\mathrm{DE} / \mathrm{DX}=0.0$ \\
\hline D1 & $\mathrm{D}(6,1,2,3)$ & -0.0702 & $-\mathrm{DE} / \mathrm{DX}=0.0$ \\
\hline D2 & $\mathrm{D}(6,1,2,7)$ & 178.407 & $-\mathrm{DE} / \mathrm{DX}=0.0$ \\
\hline D3 & $\mathrm{D}(19,1,2,3)$ & -179.492 & $-\mathrm{DE} / \mathrm{DX}=0.0$ \\
\hline D4 & $\mathrm{D}(19,1,2,7)$ & -1.0148 & $-\mathrm{DE} / \mathrm{DX}=0.0$ \\
\hline D5 & $\mathrm{D}(2,1,6,5)$ & 1.2402 & $-\mathrm{DE} / \mathrm{DX}=0.0$ \\
\hline D6 & $\mathrm{D}(2,1,6,9)$ & -179.388 & $-\mathrm{DE} / \mathrm{DX}=0.0$ \\
\hline D7 & $\mathrm{D}(19,1,6,5)$ & -179.3346 & $-\mathrm{DE} / \mathrm{DX}=0.0$ \\
\hline D8 & $\mathrm{D}(19,1,6,9)$ & 0.0372 & $-\mathrm{DE} / \mathrm{DX}=0.0$ \\
\hline D9 & $\mathrm{D}(1,2,3,4)$ & -1.8588 & $-\mathrm{DE} / \mathrm{DX}=0.0$ \\
\hline D10 & $\mathrm{D}(1,2,3,8)$ & 176.7 & $-\mathrm{DE} / \mathrm{DX}=0.0$ \\
\hline D11 & $\mathrm{D}(7,2,3,4)$ & 179.6646 & $-\mathrm{DE} / \mathrm{DX}=0.0$ \\
\hline D12 & $\mathrm{D}(7,2,3,8)$ & -1.7766 & $-\mathrm{DE} / \mathrm{DX}=0.0$ \\
\hline D13 & $\mathrm{D}(2,3,4,5)$ & 2.4893 & $-\mathrm{DE} / \mathrm{DX}=0.0$ \\
\hline D14 & $\mathrm{D}(2,3,4,10)$ & 179.5603 & $-\mathrm{DE} / \mathrm{DX}=0.0$ \\
\hline D15 & $\mathrm{D}(8,3,4,5)$ & -176.0685 & $-\mathrm{DE} / \mathrm{DX}=0.0$ \\
\hline D16 & $\mathrm{D}(8,3,4,10)$ & 1.0025 & $-\mathrm{DE} / \mathrm{DX}=0.0$ \\
\hline D17 & $\mathrm{D}(3,4,5,6)$ & -1.2809 & $-\mathrm{DE} / \mathrm{DX}=0.0$ \\
\hline D18 & $\mathrm{D}(3,4,5,18)$ & 178.3869 & $-\mathrm{DE} / \mathrm{DX}=0.0$ \\
\hline D19 & $\mathrm{D}(10,4,5,6)$ & -178.4341 & $-\mathrm{DE} / \mathrm{DX}=0.0$ \\
\hline D20 & $\mathrm{D}(10,4,5,18)$ & 1.2337 & $-\mathrm{DE} / \mathrm{DX}=0.0$ \\
\hline D21 & $\mathrm{D}(3,4,10,15)$ & 41.1096 & $-\mathrm{DE} / \mathrm{DX}=0.0$ \\
\hline D22 & $\mathrm{D}(3,4,10,17)$ & -149.3496 & $-\mathrm{DE} / \mathrm{DX}=0.0$ \\
\hline D23 & $\mathrm{D}(5,4,10,15)$ & -141.9026 & $-\mathrm{DE} / \mathrm{DX}=0.0$ \\
\hline D24 & $\mathrm{D}(5,4,10,17)$ & 27.6382 & $-\mathrm{DE} / \mathrm{DX}=0.0$ \\
\hline D25 & $\mathrm{D}(4,5,6,1)$ & -0.54 & $-\mathrm{DE} / \mathrm{DX}=0.0$ \\
\hline D26 & $\mathrm{D}(4,5,6,9)$ & -179.9169 & $-\mathrm{DE} / \mathrm{DX}=0.0$ \\
\hline D27 & $\mathrm{D}(18,5,6,1)$ & 179.7875 & $-\mathrm{DE} / \mathrm{DX}=0.0$ \\
\hline D28 & $\mathrm{D}(18,5,6,9)$ & 0.4106 & $-\mathrm{DE} / \mathrm{DX}=0.0$ \\
\hline D29 & $\mathrm{D}(4,10,15,11)$ & 0.1444 & $-\mathrm{DE} / \mathrm{DX}=0.0$ \\
\hline D30 & $\mathrm{D}(4,10,15,16)$ & 179.2115 & $-\mathrm{DE} / \mathrm{DX}=0.0$ \\
\hline D31 & $\mathrm{D}(17,10,15,11)$ & -169.814 & $-\mathrm{DE} / \mathrm{DX}=0.0$ \\
\hline D 32 & $\mathrm{D}(17,10,15,16)$ & 9.253 & $-\mathrm{DE} / \mathrm{DX}=0.0$ \\
\hline D33 & $\mathrm{D}(12,11,15,10)$ & -88.8533 & $-\mathrm{DE} / \mathrm{DX}=0.0$ \\
\hline D34 & $\mathrm{D}(12,11,15,16)$ & 92.1112 & $-\mathrm{DE} / \mathrm{DX}=0.0$ \\
\hline D35 & $\mathrm{D}(13,11,15,10)$ & 32.7737 & $-\mathrm{DE} / \mathrm{DX}=0.0$ \\
\hline D36 & $\mathrm{D}(13,11,15,16)$ & -146.2618 & $-\mathrm{DE} / \mathrm{DX}=0.0$ \\
\hline D37 & $\mathrm{D}(14,11,15,10)$ & 154.2581 & $-\mathrm{DE} / \mathrm{DX}=0.0$ \\
\hline D38 & $\mathrm{D}(14,11,15,16)$ & -24.7774 & $-\mathrm{DE} / \mathrm{DX}=0.0$ \\
\hline
\end{tabular}

CARTESIAN COORDINATES

$\begin{array}{cccccc}1 & 6 & 0 & -.068336 & .083353 & -.052404 \\ 2 & 6 & 0 & .192550 & -.548320 & 1.161301 \\ 3 & 6 & 0 & 1.498492 & -.600252 & 1.638872 \\ 4 & 6 & 0 & 2.572218 & -.064477 & .909688 \\ 5 & 6 & 0 & 2.265786 & .579005 & -.306705 \\ 6 & 6 & 0 & .962719 & .662327 & -.788224 \\ 7 & 1 & 0 & -.618484 & -.973909 & 1.740550 \\ 8 & 1 & 0 & 1.687854 & -1.044672 & 2.607738 \\ 9 & 1 & 0 & .762118 & 1.162731 & -1.727268 \\ 10 & 7 & 0 & 3.893923 & -.096605 & 1.373307 \\ 11 & 6 & 0 & 3.959822 & -2.414835 & 2.329671\end{array}$

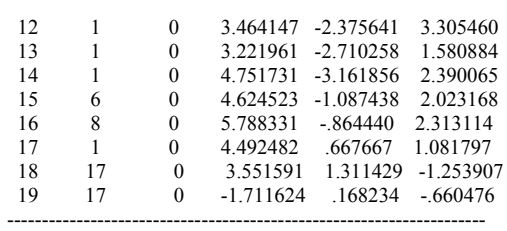

S2.6. g

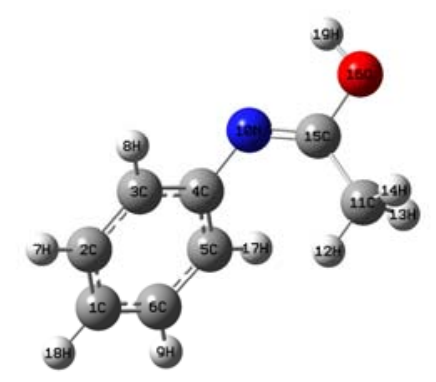

OPTIMIZED PARAMETERS

\begin{tabular}{|c|c|c|c|}
\hline R1 & $\mathrm{R}(1,2)$ & 1.3973 & $-\mathrm{DE} / \mathrm{DX}=0.0$ \\
\hline R2 & $\mathrm{R}(1,6)$ & 1.3951 & $-\mathrm{DE} / \mathrm{DX}=$ \\
\hline R3 & $\mathrm{R}(1,18)$ & 1.0857 & $-\mathrm{DE} / \mathrm{DX}=0.0$ \\
\hline R4 & $\mathrm{R}(2,3)$ & 1.3926 & $-\mathrm{DE} / \mathrm{DX}=0.0$ \\
\hline R5 & $\mathrm{R}(2,7)$ & 1.0864 & $-\mathrm{DE} / \mathrm{DX}=$ \\
\hline R6 & $\mathrm{R}(3,4)$ & 1.4045 & $-\mathrm{DE} / \mathrm{DX}=0.0$ \\
\hline R7 & $\mathrm{R}(3,8)$ & 1.0855 & $-\mathrm{DE} / \mathrm{DX}=$ \\
\hline R8 & $\mathrm{R}(4,5)$ & 1.4051 & $-\mathrm{DE} / \mathrm{DX}=0.0$ \\
\hline R9 & $\mathrm{R}(4,10)$ & 1.4065 & $-\mathrm{DE} / \mathrm{DX}=0.0$ \\
\hline R10 & $\mathrm{R}(5,6)$ & 1.3952 & $-\mathrm{DE} / \mathrm{DX}=0.0$ \\
\hline R11 & $\mathrm{R}(5,17)$ & 1.086 & $-\mathrm{DE} / \mathrm{DX}=0.0$ \\
\hline R12 & $\mathrm{R}(6,9)$ & 1.0865 & $-\mathrm{DE} / \mathrm{DX}=0.0$ \\
\hline R13 & $\mathrm{R}(10,15)$ & 1.2743 & $-\mathrm{DE} / \mathrm{DX}=0.0$ \\
\hline R14 & $\mathrm{R}(11,12)$ & 1.0892 & $-\mathrm{DE} / \mathrm{DX}=0.0$ \\
\hline R15 & $\mathrm{R}(11,13)$ & 1.0946 & $-\mathrm{DE} / \mathrm{DX}=0.0$ \\
\hline R16 & $\mathrm{R}(11,14)$ & 1.0939 & $-\mathrm{DE} / \mathrm{DX}=0.0$ \\
\hline R17 & $\mathrm{R}(11,15)$ & 1.5037 & $-\mathrm{DE} / \mathrm{DX}=0.0$ \\
\hline R18 & $\mathrm{R}(12,17)$ & 2.7454 & $-\mathrm{DE} / \mathrm{DX}=0.0$ \\
\hline R19 & $\mathrm{R}(15,16)$ & 1.3553 & $-\mathrm{DE} / \mathrm{DX}=0.0$ \\
\hline R20 & $\mathrm{R}(16,19)$ & 0.9724 & $-\mathrm{DE} / \mathrm{DX}=0.0$ \\
\hline Al & $\mathrm{A}(2,1,6)$ & 119.3402 & $-\mathrm{DE} / \mathrm{DX}=0.0$ \\
\hline A2 & $\mathrm{A}(2,1,18)$ & 120.3289 & $-\mathrm{DE} / \mathrm{DX}=0.0$ \\
\hline $\mathrm{A} 3$ & $\mathrm{~A}(6,1,18)$ & 120.3281 & $-\mathrm{DE} / \mathrm{DX}=0.0$ \\
\hline A4 & $\mathrm{A}(1,2,3)$ & 120.5074 & $-\mathrm{DE} / \mathrm{DX}=0.0$ \\
\hline A5 & $\mathrm{A}(1,2,7)$ & 120.0128 & $-\mathrm{DE} / \mathrm{DX}=$ \\
\hline A6 & $\mathrm{A}(3,2,7)$ & 119.4786 & $-\mathrm{DE} / \mathrm{DX}=0.0$ \\
\hline A7 & $\mathrm{A}(2,3,4)$ & 120.4607 & $-\mathrm{DE} / \mathrm{DX}=$ \\
\hline $\mathrm{A} 8$ & $\mathrm{~A}(2,3,8)$ & 120.9104 & $-\mathrm{DE} / \mathrm{DX}=$ \\
\hline A9 & $\mathrm{A}(4,3,8)$ & 118.6285 & $-\mathrm{DE} / \mathrm{DX}=0.0$ \\
\hline A10 & $\mathrm{A}(3,4,5)$ & 118.7817 & $-\mathrm{DE} / \mathrm{DX}=0.0$ \\
\hline A11 & $\mathrm{A}(3,4,10)$ & 118.7917 & $-\mathrm{DE} / \mathrm{DX}=0.0$ \\
\hline A12 & $\mathrm{A}(5,4,10)$ & 122.2758 & $-\mathrm{DE} / \mathrm{DX}=0.0$ \\
\hline A13 & $\mathrm{A}(4,5,6)$ & 120.4177 & $-\mathrm{DE} / \mathrm{DX}=0.0$ \\
\hline A14 & $\mathrm{A}(4,5,17)$ & 119.2378 & $-\mathrm{DE} / \mathrm{DX}=0.0$ \\
\hline A15 & $\mathrm{A}(6,5,17)$ & 120.3297 & $-\mathrm{DE} / \mathrm{DX}=0.0$ \\
\hline A16 & $\mathrm{A}(1,6,5)$ & 120.4739 & $-\mathrm{DE} / \mathrm{DX}=0.0$ \\
\hline A17 & $\mathrm{A}(1,6,9)$ & 120.0848 & $-\mathrm{DE} / \mathrm{DX}=0.0$ \\
\hline A18 & $\mathrm{A}(5,6,9)$ & 119.441 & $-\mathrm{DE} / \mathrm{DX}=0.0$ \\
\hline A19 & $\mathrm{A}(4,10,15)$ & 123.083 & $-\mathrm{DE} / \mathrm{DX}=0.0$ \\
\hline A20 & $\mathrm{A}(12,11,13)$ & 109.2295 & $-\mathrm{DE} / \mathrm{DX}=0.0$ \\
\hline A21 & $\mathrm{A}(12,11,14)$ & 109.9201 & $-\mathrm{DE} / \mathrm{DX}=0.0$ \\
\hline A22 & $\mathrm{A}(12,11,15)$ & 111.3048 & $-\mathrm{DE} / \mathrm{DX}=0.0$ \\
\hline A23 & $\mathrm{A}(13,11,14)$ & 107.172 & $-\mathrm{DE} / \mathrm{DX}=0.0$ \\
\hline A24 & $\mathrm{A}(13,11,15)$ & 110.0263 & $-\mathrm{DE} / \mathrm{DX}=0.0$ \\
\hline A25 & $\mathrm{A}(14,11,15)$ & 109.098 & $-\mathrm{DE} / \mathrm{DX}=0.0$ \\
\hline A26 & $\mathrm{A}(11,12,17)$ & 97.8043 & $-\mathrm{DE} / \mathrm{DX}=0.0$ \\
\hline A27 & $\mathrm{A}(10,15,11)$ & 130.3045 & $-\mathrm{DE} / \mathrm{DX}=0.0$ \\
\hline A28 & $\mathrm{A}(10,15,16)$ & 118.4907 & $-\mathrm{DE} / \mathrm{DX}=0.0$ \\
\hline A29 & $\mathrm{A}(11,15,16)$ & 111.1857 & $-\mathrm{DE} / \mathrm{DX}=0.0$ \\
\hline A30 & $\mathrm{A}(15,16,19)$ & 105.6646 & $-\mathrm{DE} / \mathrm{DX}=0.0$ \\
\hline A31 & $\mathrm{A}(5,17,12)$ & 79.1856 & $-\mathrm{DE} / \mathrm{DX}=0.0$ \\
\hline D1 & $\mathrm{D}(6,1,2,3)$ & -0.0624 & $-\mathrm{DE} / \mathrm{DX}=0.0$ \\
\hline D2 & $\mathrm{D}(6,1,2,7)$ & 179.5249 & $-\mathrm{DE} / \mathrm{DX}=0.0$ \\
\hline D3 & $\mathrm{D}(18,1,2,3)$ & -179.4576 & $-\mathrm{DE} / \mathrm{DX}=0.0$ \\
\hline
\end{tabular}




\begin{tabular}{|c|c|c|c|}
\hline D4 & $\mathrm{D}(18,1,2,7)$ & 0.1297 & $-\mathrm{DE} / \mathrm{DX}=0.0$ \\
\hline D5 & $\mathrm{D}(2,1,6,5)$ & 0.558 & $-\mathrm{DE} / \mathrm{DX}=0.0$ \\
\hline D6 & $\mathrm{D}(2,1,6,9)$ & -179.2344 & $-\mathrm{DE} / \mathrm{DX}=0.0$ \\
\hline D7 & $\mathrm{D}(18,1,6,5)$ & 179.9532 & $-\mathrm{DE} / \mathrm{DX}=0.0$ \\
\hline D8 & $\mathrm{D}(18,1,6,9)$ & 0.1609 & $-\mathrm{DE} / \mathrm{DX}=0.0$ \\
\hline D9 & $\mathrm{D}(1,2,3,4)$ & -1.0527 & $-\mathrm{DE} / \mathrm{DX}=0.0$ \\
\hline D10 & $\mathrm{D}(1,2,3,8)$ & 179.1599 & $-\mathrm{DE} / \mathrm{DX}=0.0$ \\
\hline D11 & $\mathrm{D}(7,2,3,4)$ & 179.3578 & $-\mathrm{DE} / \mathrm{DX}=0.0$ \\
\hline D12 & $\mathrm{D}(7,2,3,8)$ & -0.4296 & $-\mathrm{DE} / \mathrm{DX}=0.0$ \\
\hline D13 & $\mathrm{D}(2,3,4,5)$ & 1.645 & $-\mathrm{DE} / \mathrm{DX}=0.0$ \\
\hline D14 & $\mathrm{D}(2,3,4,10)$ & 177.2852 & $-\mathrm{DE} / \mathrm{DX}=0.0$ \\
\hline D15 & $\mathrm{D}(8,3,4,5)$ & -178.5627 & $-\mathrm{DE} / \mathrm{DX}=0.0$ \\
\hline D16 & $\mathrm{D}(8,3,4,10)$ & -2.9225 & $-\mathrm{DE} / \mathrm{DX}=0.0$ \\
\hline D17 & $\mathrm{D}(3,4,5,6)$ & -1.1505 & $-\mathrm{DE} / \mathrm{DX}=0.0$ \\
\hline D18 & $\mathrm{D}(3,4,5,17)$ & 177.4509 & $-\mathrm{DE} / \mathrm{DX}=0.0$ \\
\hline D19 & $\mathrm{D}(10,4,5,6)$ & -176.6311 & $-\mathrm{DE} / \mathrm{DX}=0.0$ \\
\hline D20 & $\mathrm{D}(10,4,5,17)$ & 1.9703 & $-\mathrm{DE} / \mathrm{DX}=0.0$ \\
\hline D21 & $\mathrm{D}(3,4,10,15)$ & 123.4891 & $-\mathrm{DE} / \mathrm{DX}=0.0$ \\
\hline D22 & $\mathrm{D}(5,4,10,15)$ & -61.0308 & $-\mathrm{DE} / \mathrm{DX}=0.0$ \\
\hline D23 & $\mathrm{D}(4,5,6,1)$ & 0.0577 & $-\mathrm{DE} / \mathrm{DX}=0.0$ \\
\hline D24 & $\mathrm{D}(4,5,6,9)$ & 179.8514 & $-\mathrm{DE} / \mathrm{DX}=0.0$ \\
\hline D25 & $\mathrm{D}(17,5,6,1)$ & -178.5284 & $-\mathrm{DE} / \mathrm{DX}=0.0$ \\
\hline D26 & $\mathrm{D}(17,5,6,9)$ & 1.2653 & $-\mathrm{DE} / \mathrm{DX}=0.0$ \\
\hline D27 & $\mathrm{D}(4,5,17,12)$ & 60.9704 & $-\mathrm{DE} / \mathrm{DX}=0.0$ \\
\hline D28 & $\mathrm{D}(6,5,17,12)$ & -120.4269 & $-\mathrm{DE} / \mathrm{DX}=0.0$ \\
\hline D29 & $\mathrm{D}(4,10,15,11)$ & -4.7908 & $-\mathrm{DE} / \mathrm{DX}=0.0$ \\
\hline D30 & $\mathrm{D}(4,10,15,16)$ & 176.9497 & $-\mathrm{DE} / \mathrm{DX}=0.0$ \\
\hline D31 & $\mathrm{D}(13,11,12,17)$ & -67.7757 & $-\mathrm{DE} / \mathrm{DX}=0.0$ \\
\hline D32 & $\mathrm{D}(14,11,12,17)$ & 174.9021 & $-\mathrm{DE} / \mathrm{DX}=0.0$ \\
\hline D33 & $\mathrm{D}(15,11,12,17)$ & 53.9142 & $-\mathrm{DE} / \mathrm{DX}=0.0$ \\
\hline D34 & $\mathrm{D}(12,11,15,10)$ & -5.1346 & $-\mathrm{DE} / \mathrm{DX}=0.0$ \\
\hline D35 & $\mathrm{D}(12,11,15,16)$ & 173.2248 & $-\mathrm{DE} / \mathrm{DX}=0.0$ \\
\hline D36 & $\mathrm{D}(13,11,15,10)$ & 116.0908 & $-\mathrm{DE} / \mathrm{DX}=0.0$ \\
\hline D37 & $\mathrm{D}(13,11,15,16)$ & -65.5498 & $-\mathrm{DE} / \mathrm{DX}=0.0$ \\
\hline D38 & $\mathrm{D}(14,11,15,10)$ & -126.603 & $-\mathrm{DE} / \mathrm{DX}=0.0$ \\
\hline D39 & $\mathrm{D}(14,11,15,16)$ & 51.7564 & $-\mathrm{DE} / \mathrm{DX}=0.0$ \\
\hline D40 & $\mathrm{D}(11,12,17,5)$ & -126.4115 & $-\mathrm{DE} / \mathrm{DX}=0.0$ \\
\hline D41 & $\mathrm{D}(10,15,16,19)$ & -1.0959 & $-\mathrm{DE} / \mathrm{DX}=0.0$ \\
\hline D42 & $\mathrm{D}(11,15,16,19)$ & -179.6724 & $-\mathrm{DE} / \mathrm{DX}=0.0$ \\
\hline
\end{tabular}

\section{CARTESIAN COORDINATES}

$\begin{array}{cccccc}1 & 6 & 0 & -.102592 & -.113853 & .049061 \\ 2 & 6 & 0 & .162260 & -.565557 & 1.344589 \\ 3 & 6 & 0 & 1.408254 & -.349270 & 1.927804 \\ 4 & 6 & 0 & 2.424351 & .306885 & 1.213860 \\ 5 & 6 & 0 & 2.148527 & .770434 & -.083565 \\ 6 & 6 & 0 & .894523 & .559145 & -.657454 \\ 7 & 1 & 0 & -.608801 & -1.084089 & 1.907569 \\ 8 & 1 & 0 & 1.616668 & -.685382 & 2.938663 \\ 9 & 1 & 0 & .697650 & .926511 & -1.660844 \\ 10 & 7 & 0 & 3.649960 & .558229 & 1.856501 \\ 11 & 6 & 0 & 5.080241 & -.718608 & .216951 \\ 12 & 1 & 0 & 4.169594 & -1.096802 & -.245778 \\ 13 & 1 & 0 & 5.653104 & -.152624 & -.524414 \\ 14 & 1 & 0 & 5.707986 & -1.553652 & .541353 \\ 15 & 6 & 0 & 4.769472 & .152808 & 1.402395 \\ 16 & 8 & 0 & 5.899902 & .501210 & 2.063951 \\ 17 & 1 & 0 & 2.915269 & 1.315416 & -.626339 \\ 18 & 1 & 0 & -1.077864 & -.276311 & -.399539 \\ 19 & 1 & 0 & 5.608233 & 1.052968 & 2.809659 \\ ----------------------------------------------------\end{array}$

\section{S2.7.h}

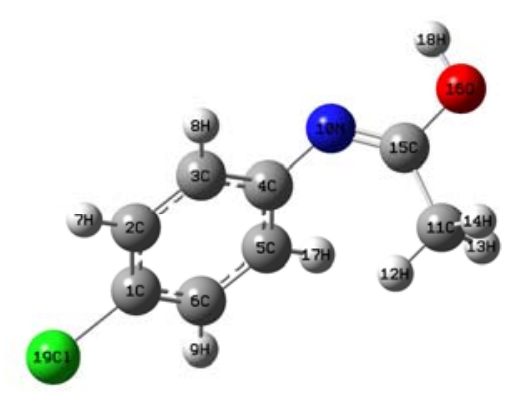

OPTIMIZED PARAMETERS

\begin{tabular}{|c|c|c|c|}
\hline R1 & $\mathrm{R}(1,2)$ & 1.3951 & $-\mathrm{DE} / \mathrm{DX}=0.0$ \\
\hline R2 & $\mathrm{R}(1,6)$ & 1.3927 & $-\mathrm{DE} / \mathrm{DX}=0.0$ \\
\hline R3 & $\mathrm{R}(1,19)$ & 1.7612 & $-\mathrm{DE} / \mathrm{DX}=0.0$ \\
\hline R4 & $\mathrm{R}(2,3)$ & 1.3917 & $-\mathrm{DE} / \mathrm{DX}=0.0$ \\
\hline R5 & $\mathrm{R}(2,7)$ & 1.0844 & $-\mathrm{DE} / \mathrm{DX}=0.0$ \\
\hline R6 & $\mathrm{R}(3,4)$ & 1.4046 & $-\mathrm{DE} / \mathrm{DX}=$ \\
\hline R7 & $\mathrm{R}(3,8)$ & 1.0851 & $-\mathrm{DE} / \mathrm{DX}=0.0$ \\
\hline R8 & $\mathrm{R}(4,5)$ & 1.4049 & $-\mathrm{DE} / \mathrm{DX}=0.0$ \\
\hline R9 & $\mathrm{R}(4,10)$ & 1.404 & $-\mathrm{DE} / \mathrm{DX}=0.0$ \\
\hline R10 & $\mathrm{R}(5,6)$ & 1.3944 & $-\mathrm{DE} / \mathrm{DX}=0.0$ \\
\hline R11 & $\mathrm{R}(5,17)$ & 1.0856 & $-\mathrm{DE} / \mathrm{DX}=0.0$ \\
\hline R12 & $R(6,9)$ & 1.0844 & $-\mathrm{DE} / \mathrm{DX}=0.0$ \\
\hline R13 & $\mathrm{R}(10,15)$ & 1.2753 & $-\mathrm{DE} / \mathrm{DX}=0.0$ \\
\hline R14 & $\mathrm{R}(11,12)$ & 1.0893 & $-\mathrm{DE} / \mathrm{DX}=0.0$ \\
\hline R15 & $\mathrm{R}(11,13)$ & 1.0946 & $-\mathrm{DE} / \mathrm{DX}=0.0$ \\
\hline R16 & $\mathrm{R}(11,14)$ & 1.0937 & $-\mathrm{DE} / \mathrm{DX}=0.0$ \\
\hline R17 & $\mathrm{R}(11,15)$ & 1.5036 & $-\mathrm{DE} / \mathrm{DX}=0.0$ \\
\hline R18 & $\mathrm{R}(12,17)$ & 2.7423 & $-\mathrm{DE} / \mathrm{DX}=0.0$ \\
\hline R19 & $\mathrm{R}(15,16)$ & 1.3535 & $-\mathrm{DE} / \mathrm{DX}=0.0$ \\
\hline R20 & $\mathrm{R}(16,18)$ & 0.9725 & $-\mathrm{DE} / \mathrm{DX}=0.0$ \\
\hline A1 & $\mathrm{A}(2,1,6)$ & 120.7844 & $-\mathrm{DE} / \mathrm{DX}=0.0$ \\
\hline A2 & $\mathrm{A}(2,1,19)$ & 119.601 & $-\mathrm{DE} / \mathrm{DX}=0.0$ \\
\hline A3 & $\mathrm{A}(6,1,19)$ & 119.6125 & $-\mathrm{DE} / \mathrm{DX}=0.0$ \\
\hline A4 & $\mathrm{A}(1,2,3)$ & 119.4197 & $-\mathrm{DE} / \mathrm{DX}=0.0$ \\
\hline A5 & $\mathrm{A}(1,2,7)$ & 120.0492 & $-\mathrm{DE} / \mathrm{DX}=0.0$ \\
\hline A6 & $\mathrm{A}(3,2,7)$ & 120.5298 & $-\mathrm{DE} / \mathrm{DX}=0.0$ \\
\hline A7 & $\mathrm{A}(2,3,4)$ & 120.9617 & $-\mathrm{DE} / \mathrm{DX}=0.0$ \\
\hline A8 & $\mathrm{A}(2,3,8)$ & 120.264 & $-\mathrm{DE} / \mathrm{DX}=0.0$ \\
\hline A9 & $\mathrm{A}(4,3,8)$ & 118.774 & $-\mathrm{DE} / \mathrm{DX}=0.0$ \\
\hline A10 & $\mathrm{A}(3,4,5)$ & 118.506 & $-\mathrm{DE} / \mathrm{DX}=0.0$ \\
\hline A11 & $\mathrm{A}(3,4,10)$ & 118.8262 & $-\mathrm{DE} / \mathrm{DX}=0.0$ \\
\hline A12 & $\mathrm{A}(5,4,10)$ & 122.5018 & $-\mathrm{DE} / \mathrm{DX}=0.0$ \\
\hline A13 & $\mathrm{A}(4,5,6)$ & 120.8955 & $-\mathrm{DE} / \mathrm{DX}=0.0$ \\
\hline A14 & $\mathrm{A}(4,5,17)$ & 119.4281 & $-\mathrm{DE} / \mathrm{DX}=0.0$ \\
\hline A15 & $\mathrm{A}(6,5,17)$ & 119.6616 & $-\mathrm{DE} / \mathrm{DX}=0.0$ \\
\hline A16 & $\mathrm{A}(1,6,5)$ & 119.4121 & $-\mathrm{DE} / \mathrm{DX}=0.0$ \\
\hline A17 & $\mathrm{A}(1,6,9)$ & 120.1085 & $-\mathrm{DE} / \mathrm{DX}=0.0$ \\
\hline A18 & $\mathrm{A}(5,6,9)$ & 120.479 & $-\mathrm{DE} / \mathrm{DX}=0.0$ \\
\hline A19 & $\mathrm{A}(4,10,15)$ & 123.2138 & $-\mathrm{DE} / \mathrm{DX}=0.0$ \\
\hline A20 & $\mathrm{A}(12,11,13)$ & 109.2137 & $-\mathrm{DE} / \mathrm{DX}=0.0$ \\
\hline A21 & $\mathrm{A}(12,11,14)$ & 109.8686 & $-\mathrm{DE} / \mathrm{DX}=0.0$ \\
\hline A 22 & $\mathrm{~A}(12,11,15)$ & 111.4329 & $-\mathrm{DE} / \mathrm{DX}=0.0$ \\
\hline A23 & $\mathrm{A}(13,11,14)$ & 107.1547 & $-\mathrm{DE} / \mathrm{DX}=0.0$ \\
\hline A24 & $\mathrm{A}(13,11,15)$ & 110.0058 & $-\mathrm{DE} / \mathrm{DX}=0.0$ \\
\hline A25 & $\mathrm{A}(14,11,15)$ & 109.0689 & $-\mathrm{DE} / \mathrm{DX}=0.0$ \\
\hline A26 & $\mathrm{A}(11,12,17)$ & 97.499 & $-\mathrm{DE} / \mathrm{DX}=0.0$ \\
\hline A27 & $\mathrm{A}(10,15,11)$ & 130.3043 & $-\mathrm{DE} / \mathrm{DX}=0.0$ \\
\hline A28 & $\mathrm{A}(10,15,16)$ & 118.4611 & $-\mathrm{DE} / \mathrm{DX}=0.0$ \\
\hline A29 & $\mathrm{A}(11,15,16)$ & 111.2157 & $-\mathrm{DE} / \mathrm{DX}=0.0$ \\
\hline $\mathrm{A} 30$ & $\mathrm{~A}(15,16,18)$ & 105.859 & $-\mathrm{DE} / \mathrm{DX}=0.0$ \\
\hline A31 & $\mathrm{A}(5,17,12)$ & 79.5607 & $-\mathrm{DE} / \mathrm{DX}=0.0$ \\
\hline D1 & $\mathrm{D}(6,1,2,3)$ & -0.1492 & $-\mathrm{DE} / \mathrm{DX}=0.0$ \\
\hline D2 & $\mathrm{D}(6,1,2,7)$ & 179.4441 & $-\mathrm{DE} / \mathrm{DX}=0.0$ \\
\hline D3 & $\mathrm{D}(19,1,2,3)$ & -179.6147 & $-\mathrm{DE} / \mathrm{DX}=0.0$ \\
\hline D4 & $\mathrm{D}(19,1,2,7)$ & -0.0214 & $-\mathrm{DE} / \mathrm{DX}=0.0$ \\
\hline D5 & $\mathrm{D}(2,1,6,5)$ & 0.6435 & $-\mathrm{DE} / \mathrm{DX}=0.0$ \\
\hline D6 & $\mathrm{D}(2,1,6,9)$ & -179.1141 & $-\mathrm{DE} / \mathrm{DX}=0.0$ \\
\hline D7 & $\mathrm{D}(19,1,6,5)$ & -179.8911 & $-\mathrm{DE} / \mathrm{DX}=0.0$ \\
\hline D8 & $\mathrm{D}(19,1,6,9)$ & 0.3513 & $-\mathrm{DE} / \mathrm{DX}=0.0$ \\
\hline D9 & $\mathrm{D}(1,2,3,4)$ & -1.0603 & $-\mathrm{DE} / \mathrm{DX}=0.0$ \\
\hline D10 & $\mathrm{D}(1,2,3,8)$ & 179.1715 & $-\mathrm{DE} / \mathrm{DX}=0.0$ \\
\hline D11 & $\mathrm{D}(7,2,3,4)$ & 179.3483 & $-\mathrm{DE} / \mathrm{DX}=0.0$ \\
\hline D12 & $\mathrm{D}(7,2,3,8)$ & -0.4199 & $-\mathrm{DE} / \mathrm{DX}=0.0$ \\
\hline D13 & $\mathrm{D}(2,3,4,5)$ & 1.7317 & $-\mathrm{DE} / \mathrm{DX}=0.0$ \\
\hline D14 & $\mathrm{D}(2,3,4,10)$ & 177.1671 & $-\mathrm{DE} / \mathrm{DX}=0.0$ \\
\hline D15 & $\mathrm{D}(8,3,4,5)$ & -178.4967 & $-\mathrm{DE} / \mathrm{DX}=0.0$ \\
\hline D16 & $\mathrm{D}(8,3,4,10)$ & -3.0612 & $-\mathrm{DE} / \mathrm{DX}=0.0$ \\
\hline D17 & $\mathrm{D}(3,4,5,6)$ & -1.2267 & $-\mathrm{DE} / \mathrm{DX}=0.0$ \\
\hline D18 & $\mathrm{D}(3,4,5,17)$ & 177.3669 & $-\mathrm{DE} / \mathrm{DX}=0.0$ \\
\hline D19 & $\mathrm{D}(10,4,5,6)$ & -176.4847 & $-\mathrm{DE} / \mathrm{DX}=0.0$ \\
\hline D20 & $\mathrm{D}(10,4,5,17)$ & 2.1089 & $-\mathrm{DE} / \mathrm{DX}=0.0$ \\
\hline D21 & $\mathrm{D}(3,4,10,15)$ & 124.4104 & $-\mathrm{DE} / \mathrm{DX}=0.0$ \\
\hline D22 & $\mathrm{D}(5,4,10,15)$ & -60.3462 & $-\mathrm{DE} / \mathrm{DX}=0.0$ \\
\hline D23 & $\mathrm{D}(4,5,6,1)$ & 0.0607 & $-\mathrm{DE} / \mathrm{DX}=0.0$ \\
\hline D24 & $\mathrm{D}(4,5,6,9)$ & 179.8174 & $-\mathrm{DE} / \mathrm{DX}=0.0$ \\
\hline D25 & $\mathrm{D}(17,5,6,1)$ & -178.5296 & $-\mathrm{DE} / \mathrm{DX}=0.0$ \\
\hline D26 & $\mathrm{D}(17,5,6,9)$ & 1.227 & $-\mathrm{DE} / \mathrm{DX}=0.0$ \\
\hline D27 & $\mathrm{D}(4,5,17,12)$ & 60.7966 & $-\mathrm{DE} / \mathrm{DX}=0.0$ \\
\hline D28 & $\mathrm{D}(6,5,17,12)$ & -120.5922 & $-\mathrm{DE} / \mathrm{DX}=0.0$ \\
\hline D29 & $\mathrm{D}(4,10,15,11)$ & -4.9637 & $-\mathrm{DE} / \mathrm{DX}=0.0$ \\
\hline D30 & $\mathrm{D}(4,10,15,16)$ & 176.7691 & $-\mathrm{DE} / \mathrm{DX}=0.0$ \\
\hline D31 & $\mathrm{D}(13,11,12,17)$ & -67.2911 & $-\mathrm{DE} / \mathrm{DX}=0.0$ \\
\hline D32 & $\mathrm{D}(14,11,12,17)$ & 175.4467 & $-\mathrm{DE} / \mathrm{DX}=0.0$ \\
\hline D33 & $\mathrm{D}(15,11,12,17)$ & 54.4458 & $-\mathrm{DE} / \mathrm{DX}=0.0$ \\
\hline D34 & $\mathrm{D}(12,11,15,10)$ & -5.7304 & $-\mathrm{DE} / \mathrm{DX}=0.0$ \\
\hline
\end{tabular}




\begin{tabular}{|c|c|c|c|}
\hline D35 & $\mathrm{D}(12,11,15,16)$ & 172.6355 & $-\mathrm{DE} / \mathrm{DX}=$ \\
\hline D36 & $\mathrm{D}(13,11,15,10)$ & 115.5461 & $-\mathrm{DE} / \mathrm{DX}=0.0$ \\
\hline D37 & $\mathrm{D}(13,11,15,16)$ & -66.088 & $-\mathrm{DE} / \mathrm{DX}=0.0$ \\
\hline D38 & $\mathrm{D}(14,11,15,10)$ & -127.1975 & $-\mathrm{DE} / \mathrm{DX}=0.0$ \\
\hline D39 & $\mathrm{D}(14,11,15,16)$ & 51.1684 & $-\mathrm{DE} / \mathrm{DX}=0.0$ \\
\hline D40 & $\mathrm{D}(11,12,17,5)$ & -126.0732 & $-\mathrm{DE} / \mathrm{DX}=0.0$ \\
\hline D41 & $\mathrm{D}(10,15,16,18)$ & -1.1719 & $-\mathrm{DE} / \mathrm{DX}=0.0$ \\
\hline D42 & $\mathrm{D}(11,15,16,18)$ & -179.7544 & $-\mathrm{DE} / \mathrm{DX}=0.0$ \\
\hline
\end{tabular}

CARTESIAN COORDINATES

$\begin{array}{cccccc}1 & 6 & 0 & -.089153 & -.088976 & .047111 \\ 2 & 6 & 0 & .150946 & -.547884 & 1.342539 \\ 3 & 6 & 0 & 1.399474 & -.339840 & 1.921039 \\ 4 & 6 & 0 & 2.426158 & .305004 & 1.211749 \\ 5 & 6 & 0 & 2.154752 & .770489 & -.085686 \\ 6 & 6 & 0 & .903414 & .575515 & -.669139\end{array}$

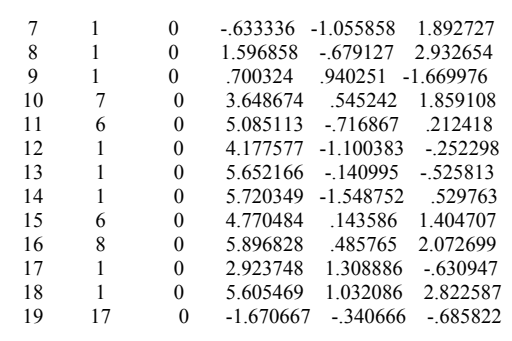

Table S3. Molecular models, Cartesian coordinates and intramolecular parameters of the optimized structures of the radicals and the respective closed shell species (theoretical level: UB3LYP/6-31G(d,p))

S3.1. $\mathrm{NH}_{2}{ }^{\bullet}$

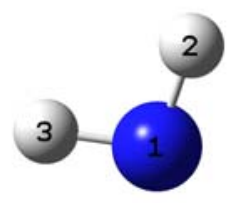

OPTIMIZED PARAMETERS

$\begin{array}{llll}\mathrm{R} 1 & \mathrm{R}(1,2) & 1.0331 & \text { calculate D2E/DX2 analytically } \\ \mathrm{R} 2 & \mathrm{R}(1,3) & 1.0331 & \text { calculate D2E/DX2 analytically } \\ \mathrm{A} 1 & \mathrm{~A}(2,1,3) & 101.8358 & \text { calculate D2E/DX2 analytically }\end{array}$

CARTESIAN COORDINATES

$\begin{array}{rrrrrr}1 & 7 & 0 & -.129607 & .000000 & -.064423 \\ 2 & 1 & 0 & .096668 & .000000 & .943603 \\ 3 & 1 & 0 & .810578 & .000000 & -.492640 \\ - & - & -\end{array}$

\section{S3.2. $\mathrm{NH}_{3}$}

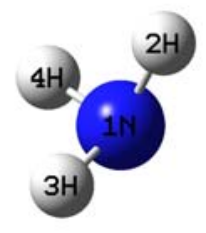

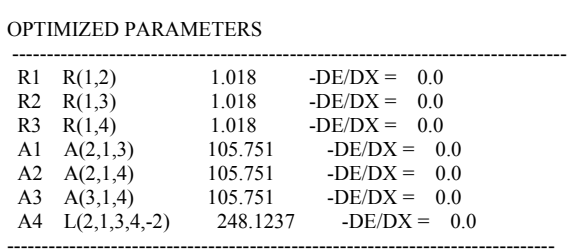

CARTESIAN COORDINATES

$\begin{array}{rrrrrr}1 & 7 & 0 & -.022941 & .039735 & -.016222 \\ 2 & 1 & 0 & .008168 & -.014148 & .999843 \\ 3 & 1 & 0 & .945384 & -.014148 & -.325580 \\ 4 & 1 & 0 & -.460439 & -.825800 & -.325580 \\ -\end{array}$

\section{S3.3. $\mathrm{CH}_{3}(\mathrm{CO}) \mathrm{NH}^{\bullet}$}

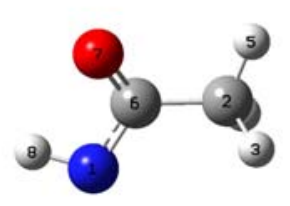

OPTIMIZED PARAMETERS

$\begin{array}{llll}\mathrm{R} 1 & \mathrm{R}(1,6) & 1.3688 & -\mathrm{DE} / \mathrm{DX}=0.0 \\ \mathrm{R} 2 & \mathrm{R}(1,8) & 1.0273 & -\mathrm{DE} / \mathrm{DX}=0.0 \\ \mathrm{R} 3 & \mathrm{R}(2,3) & 1.0948 & \mathrm{-DE} / \mathrm{DX}=0.0 \\ \mathrm{R} 4 & \mathrm{R}(2,4) & 1.094 & \text {-DE } / \mathrm{DX}=0.0 \\ \mathrm{R} 5 & \mathrm{R}(2,5) & 1.0906 & -\mathrm{DE} / \mathrm{DX}=0.0 \\ \mathrm{R} 6 & \mathrm{R}(2,6) & 1.5054 & -\mathrm{DE} / \mathrm{DX}=0.0 \\ \mathrm{R} 7 & \mathrm{R}(6,7) & 1.2398 & -\mathrm{DE} / \mathrm{DX}=0.0 \\ \mathrm{~A} 1 & \mathrm{~A}(6,1,8) & 108.5845 & \text {-DE/DX }=0.0\end{array}$




\begin{tabular}{|c|c|c|c|}
\hline A2 & $\mathrm{A}(3,2,4)$ & 107.4063 & $-\mathrm{DE} / \mathrm{DX}=$ \\
\hline A3 & $\mathrm{A}(3,2,5)$ & 109.3176 & $-\mathrm{DE} / \mathrm{DX}=$ \\
\hline A4 & $\mathrm{A}(3,2,6)$ & 109.4507 & $-\mathrm{DE} / \mathrm{DX}=$ \\
\hline A5 & $\mathrm{A}(4,2,5)$ & 110.2729 & $-\mathrm{DE} / \mathrm{DX}=$ \\
\hline A6 & $\mathrm{A}(4,2,6)$ & 110.4007 & $-\mathrm{DE} / \mathrm{DX}=$ \\
\hline A7 & $\mathrm{A}(5,2,6)$ & 109.9459 & $-\mathrm{DE} / \mathrm{DX}=$ \\
\hline A8 & $\mathrm{A}(1,6,2)$ & 117.7586 & $-\mathrm{DE} / \mathrm{DX}=$ \\
\hline A9 & $\mathrm{A}(1,6,7)$ & 118.2214 & $-\mathrm{DE} / \mathrm{DX}=$ \\
\hline A10 & $\mathrm{A}(2,6,7)$ & 123.4892 & $-\mathrm{DE} / \mathrm{DX}=$ \\
\hline D1 & $\mathrm{D}(8,1,6,2)$ & -157.7255 & $-\mathrm{DE} / \mathrm{DX}=0.0$ \\
\hline D2 & $\mathrm{D}(8,1,6,7)$ & 30.335 & $-\mathrm{DE} / \mathrm{DX}=0.0$ \\
\hline D3 & $\mathrm{D}(3,2,6,1)$ & -68.7031 & $-\mathrm{DE} / \mathrm{DX}=0.0$ \\
\hline D4 & $\mathrm{D}(3,2,6,7)$ & 102.7775 & $-\mathrm{DE} / \mathrm{DX}=0.0$ \\
\hline D5 & $\mathrm{D}(4,2,6,1)$ & 49.3197 & $-\mathrm{DE} / \mathrm{DX}=0.0$ \\
\hline D6 & $\mathrm{D}(4,2,6,7)$ & -139.1997 & $-\mathrm{DE} / \mathrm{DX}=0.0$ \\
\hline D7 & $\mathrm{D}(5,2,6,1)$ & 171.206 & $-\mathrm{DE} / \mathrm{DX}=0.0$ \\
\hline D8 & $\mathrm{D}(5,2,6,7)$ & -17.3134 & $-\mathrm{DE} / \mathrm{DX}=0.0$ \\
\hline
\end{tabular}

CARTESIAN COORDINATES

$\begin{array}{llllll}1 & 7 & 0 & .578034 & .660292 & -1.065726 \\ 2 & 6 & 0 & .305876 & .256325 & 1.347175 \\ 3 & 1 & 0 & 1.318617 & -.114921 & 1.534818 \\ 4 & 1 & 0 & .313172 & 1.334624 & 1.531689 \\ 5 & 1 & 0 & -.391011 & -.238294 & 2.024766 \\ 6 & 6 & 0 & -.076161 & -.029165 & -.080725 \\ 7 & 8 & 0 & -.832319 & -.953110 & -.414939 \\ 8 & 1 & 0 & -.006758 & .658469 & -1.910377\end{array}$




\section{S3.4. $\mathrm{CH}_{3}(\mathrm{CO}) \mathrm{NH}_{2}$}

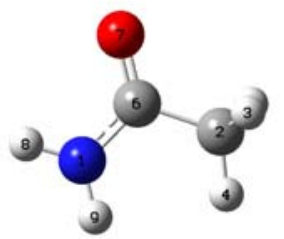

OPTIMIZED PARAMETERS

\begin{tabular}{|c|c|c|c|}
\hline R1 & $\mathrm{R}(1,6)$ & 1.3678 & $-\mathrm{DE} / \mathrm{DX}=0.0$ \\
\hline R2 & $\mathrm{R}(1,8)$ & 1.0086 & $-\mathrm{DE} / \mathrm{DX}=0.0$ \\
\hline R3 & $\mathrm{R}(1,9)$ & 1.006 & $-\mathrm{DE} / \mathrm{DX}=0.0$ \\
\hline R4 & $\mathrm{R}(2,3)$ & 1.0936 & $-\mathrm{DE} / \mathrm{DX}=0.0$ \\
\hline R5 & $\mathrm{R}(2,4)$ & 1.0933 & $-\mathrm{DE} / \mathrm{DX}=$ \\
\hline R6 & $\mathrm{R}(2,5)$ & 1.0926 & $-\mathrm{DE} / \mathrm{DX}=$ \\
\hline R7 & $\mathrm{R}(2,6)$ & 1.5225 & $-\mathrm{DE} / \mathrm{DX}=0.0$ \\
\hline R8 & $\mathrm{R}(6,7)$ & 1.2215 & $-\mathrm{DE} / \mathrm{DX}=0.0$ \\
\hline A1 & $\mathrm{A}(6,1,8)$ & 117.9808 & $-\mathrm{DE} / \mathrm{DX}=0.0$ \\
\hline A2 & $\mathrm{A}(6,1,9)$ & 122.9381 & $-\mathrm{DE} / \mathrm{DX}=0.0$ \\
\hline A3 & $\mathrm{A}(8,1,9)$ & 119.0618 & $-\mathrm{DE} / \mathrm{DX}=0.0$ \\
\hline A4 & $\mathrm{A}(3,2,4)$ & 108.9026 & $-\mathrm{DE} / \mathrm{DX}=0.0$ \\
\hline A5 & $\mathrm{A}(3,2,5)$ & 107.3454 & $-\mathrm{DE} / \mathrm{DX}=0.0$ \\
\hline A6 & $\mathrm{A}(3,2,6)$ & 108.6542 & $-\mathrm{DE} / \mathrm{DX}=0.0$ \\
\hline A7 & $\mathrm{A}(4,2,5)$ & 109.1396 & $-\mathrm{DE} / \mathrm{DX}=0.0$ \\
\hline A8 & $\mathrm{A}(4,2,6)$ & 114.0391 & $-\mathrm{DE} / \mathrm{DX}=0.0$ \\
\hline A9 & $\mathrm{A}(5,2,6)$ & 108.5525 & $-\mathrm{DE} / \mathrm{DX}=0.0$ \\
\hline A10 & $\mathrm{A}(1,6,2)$ & 115.5132 & $-\mathrm{DE} / \mathrm{DX}=0.0$ \\
\hline A11 & $\mathrm{A}(1,6,7)$ & 122.2633 & $-\mathrm{DE} / \mathrm{DX}=0.0$ \\
\hline A12 & $\mathrm{A}(2,6,7)$ & 122.2228 & $-\mathrm{DE} / \mathrm{DX}=0.0$ \\
\hline D1 & $\mathrm{D}(8,1,6,2)$ & 178.9017 & $-\mathrm{DE} / \mathrm{DX}=0.0$ \\
\hline D2 & $\mathrm{D}(8,1,6,7)$ & -0.7984 & $-\mathrm{DE} / \mathrm{DX}=0.0$ \\
\hline D3 & $\mathrm{D}(9,1,6,2)$ & 0.5144 & $-\mathrm{DE} / \mathrm{DX}=0.0$ \\
\hline D4 & $\mathrm{D}(9,1,6,7)$ & -179.1858 & $-\mathrm{DE} / \mathrm{DX}=0.0$ \\
\hline D5 & $\mathrm{D}(3,2,6,1)$ & -116.6141 & $-\mathrm{DE} / \mathrm{DX}=0.0$ \\
\hline D6 & $\mathrm{D}(3,2,6,7)$ & 63.0862 & $-\mathrm{DE} / \mathrm{DX}=0.0$ \\
\hline D7 & $\mathrm{D}(4,2,6,1)$ & 5.0525 & $-\mathrm{DE} / \mathrm{DX}=0.0$ \\
\hline D8 & $\mathrm{D}(4,2,6,7)$ & -175.2472 & $-\mathrm{DE} / \mathrm{DX}=0.0$ \\
\hline D9 & $\mathrm{D}(5,2,6,1)$ & 126.949 & $-\mathrm{DE} / \mathrm{DX}=0.0$ \\
\hline D10 & $\mathrm{D}(5,2,6,7)$ & -53.3507 & $-\mathrm{DE} / \mathrm{DX}=0.0$ \\
\hline
\end{tabular}

CARTESIAN COORDINATES

$\begin{array}{cccccc}1 & 7 & 0 & .153172 & .532570 & -1.204704 \\ 2 & 6 & 0 & .216310 & .631281 & 1.238456 \\ 3 & 1 & 0 & 1.043550 & .156145 & 1.773122 \\ 4 & 1 & 0 & .460261 & 1.683993 & 1.072041 \\ 5 & 1 & 0 & -.666364 & .565025 & 1.878994 \\ 6 & 6 & 0 & -.052139 & -.152593 & -.038875 \\ 7 & 8 & 0 & -.420658 & -1.317058 & -.023156 \\ 8 & 1 & 0 & .002678 & .043464 & -2.073820 \\ 9 & 1 & 0 & .467912 & 1.487716 & -1.229654\end{array}$

\section{S3.5. $\mathrm{CH}_{3}(\mathrm{CO}) \mathrm{PhN}^{\circ}$}

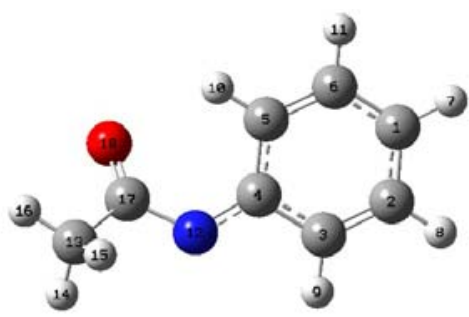

OPTIMIZED PARAMETERS

\begin{tabular}{|c|c|c|c|}
\hline R1 & $\mathrm{R}(1,2)$ & 1.4039 & $-\mathrm{DE} / \mathrm{DX}=$ \\
\hline R2 & $\mathrm{R}(1,6)$ & 1.4049 & $-\mathrm{DE} / \mathrm{DX}=$ \\
\hline R3 & $\mathrm{R}(1,7)$ & 1.0858 & $-\mathrm{DE} / \mathrm{DX}=$ \\
\hline R4 & $\mathrm{R}(2,3)$ & 1.3825 & $-\mathrm{DE} / \mathrm{DX}=$ \\
\hline R5 & $\mathrm{R}(2,8)$ & 1.0857 & $-\mathrm{DE} / \mathrm{DX}=0.0$ \\
\hline R6 & $\mathrm{R}(3,4)$ & 1.431 & $-\mathrm{DE} / \mathrm{DX}=0.0$ \\
\hline R7 & $\mathrm{R}(3,9)$ & 1.0848 & $-\mathrm{DE} / \mathrm{DX}=0.0$ \\
\hline R8 & $\mathrm{R}(4,5)$ & 1.434 & $-\mathrm{DE} / \mathrm{DX}=0.0$ \\
\hline R9 & $\mathrm{R}(4,12)$ & 1.3448 & $-\mathrm{DE} / \mathrm{DX}=0.0$ \\
\hline R10 & $\mathrm{R}(5,6)$ & 1.3828 & $-\mathrm{DE} / \mathrm{DX}=0.0$ \\
\hline R11 & $\mathrm{R}(5,10)$ & 1.0827 & $-\mathrm{DE} / \mathrm{DX}=0.0$ \\
\hline R12 & $\mathrm{R}(6,11)$ & 1.086 & $-\mathrm{DE} / \mathrm{DX}=0.0$ \\
\hline R13 & $\mathrm{R}(12,17)$ & 1.3938 & $-\mathrm{DE} / \mathrm{DX}=0.0$ \\
\hline R14 & $\mathrm{R}(13,14)$ & 1.0948 & $-\mathrm{DE} / \mathrm{DX}=0.0$ \\
\hline R15 & $\mathrm{R}(13,15)$ & 1.0948 & $-\mathrm{DE} / \mathrm{DX}=0.0$ \\
\hline R16 & $\mathrm{R}(13,16)$ & 1.0906 & $-\mathrm{DE} / \mathrm{DX}=0.0$ \\
\hline R17 & $\mathrm{R}(13,17)$ & 1.515 & $-\mathrm{DE} / \mathrm{DX}=0.0$ \\
\hline R18 & $\mathrm{R}(17,18)$ & 1.2249 & $-\mathrm{DE} / \mathrm{DX}=0.0$ \\
\hline A1 & $\mathrm{A}(2,1,6)$ & 120.1724 & $-\mathrm{DE} / \mathrm{DX}=0.0$ \\
\hline $\mathrm{A} 2$ & $\mathrm{~A}(2,1,7)$ & 119.9599 & $-\mathrm{DE} / \mathrm{DX}=0.0$ \\
\hline A3 & $\mathrm{A}(6,1,7)$ & 119.8677 & $-\mathrm{DE} / \mathrm{DX}=0.0$ \\
\hline A4 & $\mathrm{A}(1,2,3)$ & 120.013 & $-\mathrm{DE} / \mathrm{DX}=0.0$ \\
\hline A5 & $\mathrm{A}(1,2,8)$ & 119.9196 & $-\mathrm{DE} / \mathrm{DX}=0.0$ \\
\hline A6 & $\mathrm{A}(3,2,8)$ & 120.0674 & $-\mathrm{DE} / \mathrm{DX}=0.0$ \\
\hline A7 & $\mathrm{A}(2,3,4)$ & 120.9436 & $-\mathrm{DE} / \mathrm{DX}=0.0$ \\
\hline A8 & $\mathrm{A}(2,3,9)$ & 121.7642 & $-\mathrm{DE} / \mathrm{DX}=0.0$ \\
\hline A9 & $\mathrm{A}(4,3,9)$ & 117.2922 & $-\mathrm{DE} / \mathrm{DX}=0.0$ \\
\hline A10 & $\mathrm{A}(3,4,5)$ & 118.0175 & $-\mathrm{DE} / \mathrm{DX}=0.0$ \\
\hline A11 & $\mathrm{A}(3,4,12)$ & 116.6893 & $-\mathrm{DE} / \mathrm{DX}=0.0$ \\
\hline A12 & $\mathrm{A}(5,4,12)$ & 125.2579 & $-\mathrm{DE} / \mathrm{DX}=0.0$ \\
\hline A13 & $\mathrm{A}(4,5,6)$ & 120.1479 & $-\mathrm{DE} / \mathrm{DX}=0.0$ \\
\hline A14 & $\mathrm{A}(4,5,10)$ & 118.9722 & $-\mathrm{DE} / \mathrm{DX}=0.0$ \\
\hline A15 & $\mathrm{A}(6,5,10)$ & 120.8643 & $-\mathrm{DE} / \mathrm{DX}=0.0$ \\
\hline A16 & $\mathrm{A}(1,6,5)$ & 120.7054 & $-\mathrm{DE} / \mathrm{DX}=0.0$ \\
\hline A17 & $\mathrm{A}(1,6,11)$ & 119.6353 & $-\mathrm{DE} / \mathrm{DX}=0.0$ \\
\hline A18 & $\mathrm{A}(5,6,11)$ & 119.6593 & $-\mathrm{DE} / \mathrm{DX}=0.0$ \\
\hline A19 & $\mathrm{A}(4,12,17)$ & 122.6856 & $-\mathrm{DE} / \mathrm{DX}=0.0$ \\
\hline A20 & $\mathrm{A}(14,13,15)$ & 107.0345 & $-\mathrm{DE} / \mathrm{DX}=0.0$ \\
\hline A 21 & $\mathrm{~A}(14,13,16)$ & 109.6602 & $-\mathrm{DE} / \mathrm{DX}=0.0$ \\
\hline $\mathrm{A} 22$ & $\mathrm{~A}(14,13,17)$ & 109.5628 & $-\mathrm{DE} / \mathrm{DX}=0.0$ \\
\hline A23 & $\mathrm{A}(15,13,16)$ & 110.2138 & $-\mathrm{DE} / \mathrm{DX}=0.0$ \\
\hline A24 & $\mathrm{A}(15,13,17)$ & 110.7943 & $-\mathrm{DE} / \mathrm{DX}=0.0$ \\
\hline A 25 & $\mathrm{~A}(16,13,17)$ & 109.5376 & $-\mathrm{DE} / \mathrm{DX}=0.0$ \\
\hline A26 & $\mathrm{A}(12,17,13)$ & 113.3502 & $-\mathrm{DE} / \mathrm{DX}=0.0$ \\
\hline A27 & $\mathrm{A}(12,17,18)$ & 123.4547 & $-\mathrm{DE} / \mathrm{DX}=0.0$ \\
\hline A28 & $\mathrm{A}(13,17,18)$ & 122.9327 & $-\mathrm{DE} / \mathrm{DX}=0.0$ \\
\hline D1 & $\mathrm{D}(6,1,2,3)$ & -0.0498 & $-\mathrm{DE} / \mathrm{DX}=0.0$ \\
\hline D2 & $\mathrm{D}(6,1,2,8)$ & 179.9672 & $-\mathrm{DE} / \mathrm{DX}=0.0$ \\
\hline D3 & $\mathrm{D}(7,1,2,3)$ & 179.9657 & $-\mathrm{DE} / \mathrm{DX}=0.0$ \\
\hline D4 & $\mathrm{D}(7,1,2,8)$ & -0.0172 & $-\mathrm{DE} / \mathrm{DX}=0.0$ \\
\hline D5 & $\mathrm{D}(2,1,6,5)$ & 0.0947 & $-\mathrm{DE} / \mathrm{DX}=0.0$ \\
\hline D6 & $\mathrm{D}(2,1,6,11)$ & -179.8651 & $-\mathrm{DE} / \mathrm{DX}=0.0$ \\
\hline D7 & $\mathrm{D}(7,1,6,5)$ & -179.9209 & $-\mathrm{DE} / \mathrm{DX}=0.0$ \\
\hline D8 & $\mathrm{D}(7,1,6,11)$ & 0.1193 & $-\mathrm{DE} / \mathrm{DX}=0.0$ \\
\hline D9 & $\mathrm{D}(1,2,3,4)$ & 0.0639 & $-\mathrm{DE} / \mathrm{DX}=0.0$ \\
\hline D10 & $\mathrm{D}(1,2,3,9)$ & -179.8854 & $-\mathrm{DE} / \mathrm{DX}=0.0$ \\
\hline D11 & $\mathrm{D}(8,2,3,4)$ & -179.9532 & $-\mathrm{DE} / \mathrm{DX}=0.0$ \\
\hline D12 & $\mathrm{D}(8,2,3,9)$ & 0.0975 & $-\mathrm{DE} / \mathrm{DX}=0.0$ \\
\hline D13 & $\mathrm{D}(2,3,4,5)$ & -0.1176 & $-\mathrm{DE} / \mathrm{DX}=0.0$ \\
\hline D14 & $\mathrm{D}(2,3,4,12)$ & -178.0692 & $-\mathrm{DE} / \mathrm{DX}=0.0$ \\
\hline D15 & $\mathrm{D}(9,3,4,5)$ & 179.8339 & $-\mathrm{DE} / \mathrm{DX}=0.0$ \\
\hline D16 & $\mathrm{D}(9,3,4,12)$ & 1.8823 & $-\mathrm{DE} / \mathrm{DX}=0.0$ \\
\hline D17 & $\mathrm{D}(3,4,5,6)$ & 0.1605 & $-\mathrm{DE} / \mathrm{DX}=0.0$ \\
\hline D18 & $\mathrm{D}(3,4,5,10)$ & 178.7365 & $-\mathrm{DE} / \mathrm{DX}=0.0$ \\
\hline D19 & $\mathrm{D}(12,4,5,6)$ & 177.9191 & $-\mathrm{DE} / \mathrm{DX}=0.0$ \\
\hline D20 & $\mathrm{D}(12,4,5,10)$ & -3.5048 & $-\mathrm{DE} / \mathrm{DX}=0.0$ \\
\hline D21 & $\mathrm{D}(3,4,12,17)$ & -172.5401 & $-\mathrm{DE} / \mathrm{DX}=0.0$ \\
\hline D22 & $\mathrm{D}(5,4,12,17)$ & 9.6746 & $-\mathrm{DE} / \mathrm{DX}=0.0$ \\
\hline D23 & $\mathrm{D}(4,5,6,1)$ & -0.1513 & $-\mathrm{DE} / \mathrm{DX}=0.0$ \\
\hline D24 & $\mathrm{D}(4,5,6,11)$ & 179.8085 & $-\mathrm{DE} / \mathrm{DX}=0.0$ \\
\hline D25 & $\mathrm{D}(10,5,6,1)$ & -178.7 & $-\mathrm{DE} / \mathrm{DX}=0.0$ \\
\hline D26 & $\mathrm{D}(10,5,6,11)$ & 1.2597 & $-\mathrm{DE} / \mathrm{DX}=0.0$ \\
\hline D27 & $\mathrm{D}(4,12,17,13)$ & -140.0454 & $-\mathrm{DE} / \mathrm{DX}=0.0$ \\
\hline D28 & $\mathrm{D}(4,12,17,18)$ & 45.6926 & $-\mathrm{DE} / \mathrm{DX}=0.0$ \\
\hline D29 & $\mathrm{D}(14,13,17,12)$ & -64.6382 & $-\mathrm{DE} / \mathrm{DX}=0.0$ \\
\hline D30 & $\mathrm{D}(14,13,17,18)$ & 109.658 & $-\mathrm{DE} / \mathrm{DX}=0.0$ \\
\hline D31 & $\mathrm{D}(15,13,17,12)$ & 53.234 & $-\mathrm{DE} / \mathrm{DX}=0.0$ \\
\hline D32 & $\mathrm{D}(15,13,17,18)$ & -132.4698 & $-\mathrm{DE} / \mathrm{DX}=0.0$ \\
\hline D33 & $\mathrm{D}(16,13,17,12)$ & 175.0328 & $-\mathrm{DE} / \mathrm{DX}=0.0$ \\
\hline D34 & $\mathrm{D}(16,13,17,18)$ & -10.671 & $-\mathrm{DE} / \mathrm{DX}=0.0$ \\
\hline
\end{tabular}


CARTESIAN COORDINATES

$\begin{array}{cccccc}1 & 6 & 0 & -2.358161 & 1.293714 & -1.281145 \\ 2 & 6 & 0 & -2.575669 & 1.035359 & .081519 \\ 3 & 6 & 0 & -1.586241 & .435494 & .838165 \\ 4 & 6 & 0 & -.332096 & .070666 & .253431 \\ 5 & 6 & 0 & -.133927 & .343555 & -1.140374 \\ 6 & 6 & 0 & -1.135859 & .945127 & -1.879590 \\ 7 & 1 & 0 & -3.136044 & 1.764369 & -1.874721 \\ 8 & 1 & 0 & -3.521667 & 1.306953 & .539947 \\ 9 & 1 & 0 & -1.724544 & .221211 & 1.892532 \\ 10 & 1 & 0 & .816684 & .092819 & -1.593876 \\ 11 & 1 & 0 & -.976122 & 1.150977 & -2.933835 \\ 12 & 7 & 0 & .553392 & -.543726 & 1.057733 \\ 13 & 6 & 0 & 2.357468 & -2.172263 & 1.128671 \\ 14 & 1 & 0 & 2.403518 & -2.191791 & 2.222335 \\ 15 & 1 & 0 & 1.665822 & -2.963856 & .822594 \\ 16 & 1 & 0 & 3.349727 & -2.358596 & .716263 \\ 17 & 6 & 0 & 1.862721 & -.817139 & .665878 \\ 18 & 8 & 0 & 2.582433 & -.002886 & .100663 \\ \end{array}$

\section{S3.6. $\mathrm{CH}_{3}(\mathrm{CO}) \mathrm{PhNH}$}

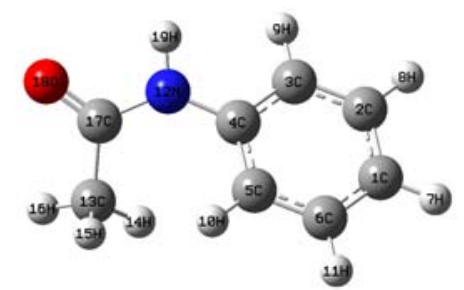

OPTIMIZED PARAMETERS

\begin{tabular}{|c|c|c|c|}
\hline R1 & $\mathrm{R}(1,2)$ & 1.3963 & $-\mathrm{DE} / \mathrm{DX}=0.0$ \\
\hline R2 & $\mathrm{R}(1,6)$ & 1.3948 & $-\mathrm{DE} / \mathrm{DX}=$ \\
\hline R3 & $\mathrm{R}(1,7)$ & 1.0854 & $-\mathrm{DE} / \mathrm{DX}=0.0$ \\
\hline R4 & $\mathrm{R}(2,3)$ & 1.3925 & $-\mathrm{DE} / \mathrm{DX}=0.0$ \\
\hline R5 & $\mathrm{R}(2,8)$ & 1.086 & $-\mathrm{DE} / \mathrm{DX}=0.0$ \\
\hline R6 & $\mathrm{R}(3,4)$ & 1.4036 & $-\mathrm{DE} / \mathrm{DX}=0.0$ \\
\hline R7 & $\mathrm{R}(3,9)$ & 1.0866 & $-\mathrm{DE} / \mathrm{DX}=0.0$ \\
\hline R8 & $\mathrm{R}(4,5)$ & 1.4022 & $-\mathrm{DE} / \mathrm{DX}=0.0$ \\
\hline R9 & $\mathrm{R}(4,12)$ & 1.4132 & $-\mathrm{DE} / \mathrm{DX}=0.0$ \\
\hline R10 & $\mathrm{R}(5,6)$ & 1.3948 & $-\mathrm{DE} / \mathrm{DX}=0.0$ \\
\hline R11 & $\mathrm{R}(5,10)$ & 1.0835 & $-\mathrm{DE} / \mathrm{DX}=0.0$ \\
\hline R12 & $\mathrm{R}(6,11)$ & 1.0861 & $-\mathrm{DE} / \mathrm{DX}=0.0$ \\
\hline R13 & $\mathrm{R}(12,17)$ & 1.3856 & $-\mathrm{DE} / \mathrm{DX}=0.0001$ \\
\hline R14 & $\mathrm{R}(12,19)$ & 1.0129 & $-\mathrm{DE} / \mathrm{DX}=0.0$ \\
\hline R15 & $\mathrm{R}(13,14)$ & 1.0919 & $-\mathrm{DE} / \mathrm{DX}=0.0$ \\
\hline R16 & $\mathrm{R}(13,15)$ & 1.0951 & $-\mathrm{DE} / \mathrm{DX}=$ \\
\hline R17 & $\mathrm{R}(13,16)$ & 1.0904 & $-\mathrm{DE} / \mathrm{DX}=0.0$ \\
\hline R18 & $\mathrm{R}(13,17)$ & 1.5167 & $-\mathrm{DE} / \mathrm{DX}=0.0$ \\
\hline R19 & $\mathrm{R}(17,18)$ & 1.2226 & $-\mathrm{DE} / \mathrm{DX}=$ \\
\hline A1 & $\mathrm{A}(2,1,6)$ & 119.2962 & $-\mathrm{DE} / \mathrm{DX}=0.0$ \\
\hline A2 & $\mathrm{A}(2,1,7)$ & 120.3534 & $-\mathrm{DE} / \mathrm{DX}=0.0$ \\
\hline A3 & $\mathrm{A}(6,1,7)$ & 120.3483 & $-\mathrm{DE} / \mathrm{DX}=0.0$ \\
\hline A4 & $\mathrm{A}(1,2,3)$ & 120.3727 & $-\mathrm{DE} / \mathrm{DX}=0.0$ \\
\hline A5 & $\mathrm{A}(1,2,8)$ & 120.1822 & $-\mathrm{DE} / \mathrm{DX}=0.0$ \\
\hline A6 & $\mathrm{A}(3,2,8)$ & 119.4415 & $-\mathrm{DE} / \mathrm{DX}=0.0$ \\
\hline A7 & $\mathrm{A}(2,3,4)$ & 120.4654 & $-\mathrm{DE} / \mathrm{DX}=0.0$ \\
\hline A8 & $\mathrm{A}(2,3,9)$ & 120.2798 & $-\mathrm{DE} / \mathrm{DX}=0.0$ \\
\hline A9 & $\mathrm{A}(4,3,9)$ & 119.252 & $-\mathrm{DE} / \mathrm{DX}=0.0$ \\
\hline A10 & $\mathrm{A}(3,4,5)$ & 119.0455 & $-\mathrm{DE} / \mathrm{DX}=0.0$ \\
\hline A11 & $\mathrm{A}(3,4,12)$ & 118.4218 & $-\mathrm{DE} / \mathrm{DX}=0.0$ \\
\hline A12 & $\mathrm{A}(5,4,12)$ & 122.4784 & $-\mathrm{DE} / \mathrm{DX}=0.0$ \\
\hline A13 & $\mathrm{A}(4,5,6)$ & 120.0753 & $-\mathrm{DE} / \mathrm{DX}=0.0$ \\
\hline A14 & $\mathrm{A}(4,5,10)$ & 120.0171 & $-\mathrm{DE} / \mathrm{DX}=0.0$ \\
\hline A15 & $\mathrm{A}(6,5,10)$ & 119.89 & $-\mathrm{DE} / \mathrm{DX}=0.0$ \\
\hline A16 & $\mathrm{A}(1,6,5)$ & 120.7139 & $-\mathrm{DE} / \mathrm{DX}=0.0$ \\
\hline A17 & $\mathrm{A}(1,6,11)$ & 120.0704 & $-\mathrm{DE} / \mathrm{DX}=0.0$ \\
\hline A18 & $\mathrm{A}(5,6,11)$ & 119.2023 & $-\mathrm{DE} / \mathrm{DX}=0.0$ \\
\hline A19 & $\mathrm{A}(4,12,17)$ & 131.9169 & $-\mathrm{DE} / \mathrm{DX}=0.0$ \\
\hline $\mathrm{A} 20$ & $\mathrm{~A}(4,12,19)$ & 116.5135 & $-\mathrm{DE} / \mathrm{DX}=0.0$ \\
\hline A21 & $\mathrm{A}(17,12,19)$ & 111.0615 & $-\mathrm{DE} / \mathrm{DX}=0.0$ \\
\hline A22 & $\mathrm{A}(14,13,15)$ & 108.1642 & $-\mathrm{DE} / \mathrm{DX}=$ \\
\hline
\end{tabular}

\begin{tabular}{|c|c|c|c|}
\hline A23 & $\mathrm{A}(14,13,16)$ & 110.2446 & $-\mathrm{DE} / \mathrm{DX}=$ \\
\hline A24 & $\mathrm{A}(14,13,17)$ & 113.1073 & $-\mathrm{DE} / \mathrm{DX}=$ \\
\hline A25 & $\mathrm{A}(15,13,16)$ & 107.8117 & $-\mathrm{DE} / \mathrm{DX}=0.0$ \\
\hline A26 & $\mathrm{A}(15,13,17)$ & 110.239 & $-\mathrm{DE} / \mathrm{DX}=0.0$ \\
\hline A27 & $\mathrm{A}(16,13,17)$ & 107.1555 & $-\mathrm{DE} / \mathrm{DX}=0.0$ \\
\hline A28 & $\mathrm{A}(12,17,13)$ & 118.8315 & $-\mathrm{DE} / \mathrm{DX}=$ \\
\hline A29 & $\mathrm{A}(12,17,18)$ & 119.2668 & $-\mathrm{DE} / \mathrm{DX}=0.0$ \\
\hline $\mathrm{A} 30$ & $\mathrm{~A}(13,17,18)$ & 121.8971 & $-\mathrm{DE} / \mathrm{DX}=0.0$ \\
\hline D1 & $\mathrm{D}(6,1,2,3)$ & -1.0538 & $-\mathrm{DE} / \mathrm{DX}=0.0$ \\
\hline D2 & $\mathrm{D}(6,1,2,8)$ & 179.6402 & $-\mathrm{DE} / \mathrm{DX}=0.0$ \\
\hline D3 & $\mathrm{D}(7,1,2,3)$ & 179.4726 & $-\mathrm{DE} / \mathrm{DX}=0.0$ \\
\hline D4 & $\mathrm{D}(7,1,2,8)$ & 0.1665 & $-\mathrm{DE} / \mathrm{DX}=0.0$ \\
\hline D5 & $\mathrm{D}(2,1,6,5)$ & -0.1436 & $-\mathrm{DE} / \mathrm{DX}=0.0$ \\
\hline D6 & $\mathrm{D}(2,1,6,11)$ & -178.7985 & $-\mathrm{DE} / \mathrm{DX}=0.0$ \\
\hline D7 & $\mathrm{D}(7,1,6,5)$ & 179.3301 & $-\mathrm{DE} / \mathrm{DX}=0.0$ \\
\hline D8 & $\mathrm{D}(7,1,6,11)$ & 0.6752 & $-\mathrm{DE} / \mathrm{DX}=0.0$ \\
\hline D9 & $\mathrm{D}(1,2,3,4)$ & 0.7557 & $-\mathrm{DE} / \mathrm{DX}=0.0$ \\
\hline D10 & $\mathrm{D}(1,2,3,9)$ & -178.6218 & $-\mathrm{DE} / \mathrm{DX}=0.0$ \\
\hline D11 & $\mathrm{D}(8,2,3,4)$ & -179.9331 & $-\mathrm{DE} / \mathrm{DX}=0.0$ \\
\hline D12 & $\mathrm{D}(8,2,3,9)$ & 0.6893 & $-\mathrm{DE} / \mathrm{DX}=0.0$ \\
\hline D13 & $\mathrm{D}(2,3,4,5)$ & 0.7339 & $-\mathrm{DE} / \mathrm{DX}=0.0$ \\
\hline D14 & $\mathrm{D}(2,3,4,12)$ & 178.1208 & $-\mathrm{DE} / \mathrm{DX}=0.0$ \\
\hline D15 & $\mathrm{D}(9,3,4,5)$ & -179.8822 & $-\mathrm{DE} / \mathrm{DX}=0.0$ \\
\hline D16 & $\mathrm{D}(9,3,4,12)$ & -2.4953 & $-\mathrm{DE} / \mathrm{DX}=0.0$ \\
\hline D17 & $\mathrm{D}(3,4,5,6)$ & -1.9195 & $-\mathrm{DE} / \mathrm{DX}=0.0$ \\
\hline D18 & $\mathrm{D}(3,4,5,10)$ & 176.5537 & $-\mathrm{DE} / \mathrm{DX}=0.0$ \\
\hline D19 & $\mathrm{D}(12,4,5,6)$ & -179.1952 & $-\mathrm{DE} / \mathrm{DX}=0.0$ \\
\hline D20 & $\mathrm{D}(12,4,5,10)$ & -0.722 & $-\mathrm{DE} / \mathrm{DX}=0.0$ \\
\hline D21 & $\mathrm{D}(3,4,12,17)$ & 139.1587 & $-\mathrm{DE} / \mathrm{DX}=0.0$ \\
\hline D22 & $\mathrm{D}(3,4,12,19)$ & -31.8068 & $-\mathrm{DE} / \mathrm{DX}=0.0$ \\
\hline D23 & $\mathrm{D}(5,4,12,17)$ & -43.5493 & $-\mathrm{DE} / \mathrm{DX}=0.0$ \\
\hline D24 & $\mathrm{D}(5,4,12,19)$ & 145.4852 & $-\mathrm{DE} / \mathrm{DX}=0.0$ \\
\hline D25 & $\mathrm{D}(4,5,6,1)$ & 1.6412 & $-\mathrm{DE} / \mathrm{DX}=0.0$ \\
\hline D26 & $\mathrm{D}(4,5,6,11)$ & -179.6924 & $-\mathrm{DE} / \mathrm{DX}=0.0$ \\
\hline D27 & $\mathrm{D}(10,5,6,1)$ & -176.834 & $-\mathrm{DE} / \mathrm{DX}=0.0$ \\
\hline D28 & $\mathrm{D}(10,5,6,11)$ & 1.8324 & $-\mathrm{DE} / \mathrm{DX}=0.0$ \\
\hline D29 & $\mathrm{D}(4,12,17,13)$ & 1.0296 & $-\mathrm{DE} / \mathrm{DX}=0.0$ \\
\hline D30 & $\mathrm{D}(4,12,17,18)$ & -178.1997 & $-\mathrm{DE} / \mathrm{DX}=0.0$ \\
\hline D31 & $\mathrm{D}(19,12,17,13)$ & 172.3695 & $-\mathrm{DE} / \mathrm{DX}=0.0$ \\
\hline D32 & $\mathrm{D}(19,12,17,18)$ & -6.8598 & $-\mathrm{DE} / \mathrm{DX}=0.0$ \\
\hline D33 & $\mathrm{D}(14,13,17,12)$ & -32.7901 & $-\mathrm{DE} / \mathrm{DX}=0.0$ \\
\hline D34 & $\mathrm{D}(14,13,17,18)$ & 146.4179 & $-\mathrm{DE} / \mathrm{DX}=0.0$ \\
\hline D35 & $\mathrm{D}(15,13,17,12)$ & 88.4453 & $-\mathrm{DE} / \mathrm{DX}=0.0$ \\
\hline D36 & $\mathrm{D}(15,13,17,18)$ & -92.3466 & $-\mathrm{DE} / \mathrm{DX}=0.0$ \\
\hline D37 & $\mathrm{D}(16,13,17,12)$ & -154.4885 & $-\mathrm{DE} / \mathrm{DX}=0.0$ \\
\hline D38 & $\mathrm{D}(16,13,17,18)$ & 24.7196 & $-\mathrm{DE} / \mathrm{DX}=0.0$ \\
\hline
\end{tabular}

CARTESIAN COORDINATES

$\begin{array}{cccccc}1 & 6 & 0 & -2.574612 & .644480 & -1.403374 \\ 2 & 6 & 0 & -2.556218 & 1.012298 & -.056526 \\ 3 & 6 & 0 & -1.389134 & .884905 & .692231 \\ 4 & 6 & 0 & -.212649 & .400385 & .099504 \\ 5 & 6 & 0 & -.225538 & .061271 & -1.260973 \\ 6 & 6 & 0 & -1.405488 & .168728 & -1.997001 \\ 7 & 1 & 0 & -3.486765 & .735892 & -1.984503 \\ 8 & 1 & 0 & -3.456729 & 1.390021 & .418647 \\ 9 & 1 & 0 & -1.382401 & 1.150543 & 1.745854 \\ 10 & 1 & 0 & .687089 & -.261080 & -1.747888 \\ 11 & 1 & 0 & -1.400580 & -.102493 & -3.048645 \\ 12 & 7 & 0 & .957226 & .326627 & .888946 \\ 13 & 6 & 0 & 1.869482 & -1.860756 & .094897 \\ 14 & 1 & 0 & .843362 & -2.175071 & -.106581 \\ 15 & 1 & 0 & 2.362589 & -1.669916 & -.864064 \\ 16 & 1 & 0 & 2.416499 & -2.658469 & .598319 \\ 17 & 6 & 0 & 1.952723 & -.632797 & .981227 \\ 18 & 8 & 0 & 2.863217 & -.474337 & 1.781692 \\ 19 & 1 & 0 & 1.059219 & 1.027803 & 1.612795\end{array}$


$\mathrm{S} 3.7 . \mathrm{Ph}_{2} \mathrm{~N}^{\circ}$

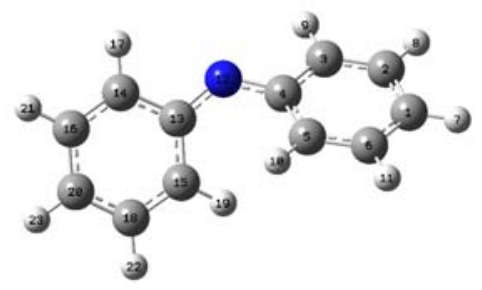

OPTIMIZED PARAMETERS

\begin{tabular}{|c|c|c|c|}
\hline R1 & $\mathrm{R}(1,2)$ & 1.4011 & $-\mathrm{DE} / \mathrm{DX}=0.0$ \\
\hline R2 & $\mathrm{R}(1,6)$ & 1.3995 & $-\mathrm{DE} / \mathrm{DX}=0.0$ \\
\hline R3 & $\mathrm{R}(1,7)$ & 1.0858 & $-\mathrm{DE} / \mathrm{DX}=$ \\
\hline R4 & $\mathrm{R}(2,3)$ & 1.3862 & $-\mathrm{DE} / \mathrm{DX}=0.0$ \\
\hline R5 & $\mathrm{R}(2,8)$ & 1.0861 & $-\mathrm{DE} / \mathrm{DX}=0.0$ \\
\hline R6 & $\mathrm{R}(3,4)$ & 1.4215 & $-\mathrm{DE} / \mathrm{DX}=0.0$ \\
\hline R7 & $\mathrm{R}(3,9)$ & 1.085 & $-\mathrm{DE} / \mathrm{DX}=0.0$ \\
\hline R8 & $\mathrm{R}(4,5)$ & 1.4234 & $-\mathrm{DE} / \mathrm{DX}=0.0$ \\
\hline R9 & $\mathrm{R}(4,12)$ & 1.3677 & $-\mathrm{DE} / \mathrm{DX}=0.0$ \\
\hline R10 & $\mathrm{R}(5,6)$ & 1.3891 & $-\mathrm{DE} / \mathrm{DX}=0.0$ \\
\hline R11 & $\mathrm{R}(5,10)$ & 1.0841 & $-\mathrm{DE} / \mathrm{DX}=0.0$ \\
\hline R12 & $\mathrm{R}(6,11)$ & 1.0864 & $-\mathrm{DE} / \mathrm{DX}=0.0$ \\
\hline R13 & $\mathrm{R}(12,13)$ & 1.3678 & $-\mathrm{DE} / \mathrm{DX}=0.0$ \\
\hline R14 & $\mathrm{R}(13,14)$ & 1.4215 & $-\mathrm{DE} / \mathrm{DX}=$ \\
\hline R15 & $\mathrm{R}(13,15)$ & 1.4233 & $-\mathrm{DE} / \mathrm{DX}=$ \\
\hline R16 & $\mathrm{R}(14,16)$ & 1.3862 & $-\mathrm{DE} / \mathrm{DX}=0.0$ \\
\hline R17 & $\mathrm{R}(14,17)$ & 1.085 & $-\mathrm{DE} / \mathrm{DX}=0.0$ \\
\hline R18 & $\mathrm{R}(15,18)$ & 1.3891 & $-\mathrm{DE} / \mathrm{DX}=0.0$ \\
\hline R19 & $\mathrm{R}(15,19)$ & 1.0841 & $-\mathrm{DE} / \mathrm{DX}=$ \\
\hline R20 & $\mathrm{R}(16,20)$ & 1.4011 & $-\mathrm{DE} / \mathrm{DX}=$ \\
\hline R21 & $\mathrm{R}(16,21)$ & 1.0861 & $-\mathrm{DE} / \mathrm{DX}=$ \\
\hline R22 & $\mathrm{R}(18,20)$ & 1.3994 & $-\mathrm{DE} / \mathrm{DX}=$ \\
\hline R23 & $\mathrm{R}(18,22)$ & 1.0864 & $-\mathrm{DE} / \mathrm{DX}=$ \\
\hline R24 & $\mathrm{R}(20,23)$ & 1.0857 & $-\mathrm{DE} / \mathrm{DX}=$ \\
\hline A1 & $\mathrm{A}(2,1,6)$ & 119.6435 & $-\mathrm{DE} / \mathrm{DX}=0.0$ \\
\hline A2 & $\mathrm{A}(2,1,7)$ & 120.2151 & $-\mathrm{DE} / \mathrm{DX}=0.0$ \\
\hline A3 & $\mathrm{A}(6,1,7)$ & 120.1307 & $-\mathrm{DE} / \mathrm{DX}=0.0$ \\
\hline A4 & $\mathrm{A}(1,2,3)$ & 120.2478 & $-\mathrm{DE} / \mathrm{DX}=0.0$ \\
\hline A5 & $\mathrm{A}(1,2,8)$ & 119.9497 & $-\mathrm{DE} / \mathrm{DX}=0.0$ \\
\hline A6 & $\mathrm{A}(3,2,8)$ & 119.7966 & $-\mathrm{DE} / \mathrm{DX}=0.0$ \\
\hline A7 & $\mathrm{A}(2,3,4)$ & 121.0761 & $-\mathrm{DE} / \mathrm{DX}=0.0$ \\
\hline A8 & $\mathrm{A}(2,3,9)$ & 121.4616 & $-\mathrm{DE} / \mathrm{DX}=0.0$ \\
\hline A9 & $\mathrm{A}(4,3,9)$ & 117.452 & $-\mathrm{DE} / \mathrm{DX}=0.0$ \\
\hline A10 & $\mathrm{A}(3,4,5)$ & 117.7608 & $-\mathrm{DE} / \mathrm{DX}=0.0$ \\
\hline A11 & $\mathrm{A}(3,4,12)$ & 116.4211 & $-\mathrm{DE} / \mathrm{DX}=0.0$ \\
\hline A12 & $\mathrm{A}(5,4,12)$ & 125.6541 & $-\mathrm{DE} / \mathrm{DX}=0.0$ \\
\hline A13 & $\mathrm{A}(4,5,6)$ & 120.5125 & $-\mathrm{DE} / \mathrm{DX}=0.0$ \\
\hline A14 & $\mathrm{A}(4,5,10)$ & 119.3657 & $-\mathrm{DE} / \mathrm{DX}=0.0$ \\
\hline A15 & $\mathrm{A}(6,5,10)$ & 120.0513 & $-\mathrm{DE} / \mathrm{DX}=0.0$ \\
\hline A16 & $\mathrm{A}(1,6,5)$ & 120.6883 & $-\mathrm{DE} / \mathrm{DX}=0.0$ \\
\hline A17 & $\mathrm{A}(1,6,11)$ & 119.8172 & $-\mathrm{DE} / \mathrm{DX}=0.0$ \\
\hline A18 & $\mathrm{A}(5,6,11)$ & 119.4926 & $-\mathrm{DE} / \mathrm{DX}=0.0$ \\
\hline A19 & $\mathrm{A}(4,12,13)$ & 123.132 & $-\mathrm{DE} / \mathrm{DX}=0.0001$ \\
\hline $\mathrm{A} 20$ & $\mathrm{~A}(12,13,14)$ & 116.4228 & $-\mathrm{DE} / \mathrm{DX}=0.0$ \\
\hline A21 & $\mathrm{A}(12,13,15)$ & 125.6479 & $-\mathrm{DE} / \mathrm{DX}=0.0$ \\
\hline A22 & $\mathrm{A}(14,13,15)$ & 117.7639 & $-\mathrm{DE} / \mathrm{DX}=$ \\
\hline A23 & $\mathrm{A}(13,14,16)$ & 121.0748 & $-\mathrm{DE} / \mathrm{DX}=0.0$ \\
\hline A24 & $\mathrm{A}(13,14,17)$ & 117.4537 & $-\mathrm{DE} / \mathrm{DX}=0.0$ \\
\hline A25 & $\mathrm{A}(16,14,17)$ & 121.4611 & $-\mathrm{DE} / \mathrm{DX}=$ \\
\hline A26 & $\mathrm{A}(13,15,18)$ & 120.5116 & $-\mathrm{DE} / \mathrm{DX}=$ \\
\hline A27 & $\mathrm{A}(13,15,19)$ & 119.3696 & $-\mathrm{DE} / \mathrm{DX}=$ \\
\hline A28 & $\mathrm{A}(18,15,19)$ & 120.0484 & $-\mathrm{DE} / \mathrm{DX}=0.0$ \\
\hline A29 & $\mathrm{A}(14,16,20)$ & 120.2478 & $-\mathrm{DE} / \mathrm{DX}=0.0$ \\
\hline A30 & $\mathrm{A}(14,16,21)$ & 119.7949 & $-\mathrm{DE} / \mathrm{DX}=$ \\
\hline A31 & $\mathrm{A}(20,16,21)$ & 119.9513 & $-\mathrm{DE} / \mathrm{DX}=0.0$ \\
\hline A32 & $\mathrm{A}(15,18,20)$ & 120.6883 & $-\mathrm{DE} / \mathrm{DX}=0.0$ \\
\hline A33 & $\mathrm{A}(15,18,22)$ & 119.4916 & $-\mathrm{DE} / \mathrm{DX}=$ \\
\hline A34 & $\mathrm{A}(20,18,22)$ & 119.8183 & $-\mathrm{DE} / \mathrm{DX}=0.0$ \\
\hline A35 & $\mathrm{A}(16,20,18)$ & 119.6423 & $-\mathrm{DE} / \mathrm{DX}=0.0$ \\
\hline A36 & $\mathrm{A}(16,20,23)$ & 120.2169 & $-\mathrm{DE} / \mathrm{DX}=0.0$ \\
\hline A37 & $\mathrm{A}(18,20,23)$ & 120.13 & $-\mathrm{DE} / \mathrm{DX}=0.0$ \\
\hline D1 & $\mathrm{D}(6,1,2,3)$ & -0.1025 & $-\mathrm{DE} / \mathrm{DX}=0.0$ \\
\hline D2 & $\mathrm{D}(6,1,2,8)$ & 179.0073 & $-\mathrm{DE} / \mathrm{DX}=0.0$ \\
\hline D3 & $\mathrm{D}(7,1,2,3)$ & -178.9129 & $-\mathrm{DE} / \mathrm{DX}=0.0$ \\
\hline D4 & $\mathrm{D}(7,1,2,8)$ & 0.1969 & $-\mathrm{DE} / \mathrm{DX}=0.0$ \\
\hline D5 & $\mathrm{D}(2,1,6,5)$ & 1.5767 & $-\mathrm{DE} / \mathrm{DX}=0.0$ \\
\hline D6 & $\mathrm{D}(2,1,6,11)$ & -177.9249 & $-\mathrm{DE} / \mathrm{DX}=0.0$ \\
\hline
\end{tabular}

\begin{tabular}{|c|c|c|c|}
\hline D7 & $\mathrm{D}(7,1,6,5)$ & -179.6119 & $-\mathrm{DE} / \mathrm{DX}=0.0$ \\
\hline D8 & $\mathrm{D}(7,1,6,11)$ & 0.8865 & $-\mathrm{DE} / \mathrm{DX}=0.0$ \\
\hline D9 & $\mathrm{D}(1,2,3,4)$ & -2.2498 & $-\mathrm{DE} / \mathrm{DX}=0.0$ \\
\hline D10 & $\mathrm{D}(1,2,3,9)$ & 178.9495 & $-\mathrm{DE} / \mathrm{DX}=0.0$ \\
\hline D11 & $\mathrm{D}(8,2,3,4)$ & 178.639 & $-\mathrm{DE} / \mathrm{DX}=$ \\
\hline D12 & $\mathrm{D}(8,2,3,9)$ & -0.1617 & $-\mathrm{DE} / \mathrm{DX}=0.0$ \\
\hline D13 & $\mathrm{D}(2,3,4,5)$ & 3.0533 & $-\mathrm{DE} / \mathrm{DX}=0.0$ \\
\hline D14 & $\mathrm{D}(2,3,4,12)$ & 178.6641 & $-\mathrm{DE} / \mathrm{DX}=0.0$ \\
\hline D15 & $\mathrm{D}(9,3,4,5)$ & -178.0995 & $-\mathrm{DE} / \mathrm{DX}=$ \\
\hline D16 & $\mathrm{D}(9,3,4,12)$ & -2.4887 & $-\mathrm{DE} / \mathrm{DX}=0.0$ \\
\hline D17 & $\mathrm{D}(3,4,5,6)$ & -1.5726 & $-\mathrm{DE} / \mathrm{DX}=0.0$ \\
\hline D18 & $\mathrm{D}(3,4,5,10)$ & 175.3763 & $-\mathrm{DE} / \mathrm{DX}=0.0$ \\
\hline D19 & $\mathrm{D}(12,4,5,6)$ & -176.7341 & $-\mathrm{DE} / \mathrm{DX}=$ \\
\hline D20 & $\mathrm{D}(12,4,5,10)$ & 0.2148 & $-\mathrm{DE} / \mathrm{DX}=0.0$ \\
\hline D21 & $\mathrm{D}(3,4,12,13)$ & 156.8872 & $-\mathrm{DE} / \mathrm{DX}=0.0$ \\
\hline D22 & $\mathrm{D}(5,4,12,13)$ & -27.8937 & $-\mathrm{DE} / \mathrm{DX}=0.0$ \\
\hline D23 & $\mathrm{D}(4,5,6,1)$ & -0.7022 & $-\mathrm{DE} / \mathrm{DX}=0.0$ \\
\hline D24 & $\mathrm{D}(4,5,6,11)$ & 178.8011 & $-\mathrm{DE} / \mathrm{DX}=0.0$ \\
\hline D25 & $\mathrm{D}(10,5,6,1)$ & -177.6302 & $-\mathrm{DE} / \mathrm{DX}=0.0$ \\
\hline D26 & $\mathrm{D}(10,5,6,11)$ & 1.8731 & $-\mathrm{DE} / \mathrm{DX}=0.0$ \\
\hline D27 & $\mathrm{D}(4,12,13,14)$ & 156.8658 & $-\mathrm{DE} / \mathrm{DX}=0.0$ \\
\hline D28 & $\mathrm{D}(4,12,13,15)$ & -27.9349 & $-\mathrm{DE} / \mathrm{DX}=0.0$ \\
\hline D29 & $\mathrm{D}(12,13,14,16)$ & 178.6544 & $-\mathrm{DE} / \mathrm{DX}=0.0$ \\
\hline D30 & $\mathrm{D}(12,13,14,17)$ & -2.5 & $-\mathrm{DE} / \mathrm{DX}=0.0$ \\
\hline D31 & $\mathrm{D}(15,13,14,16)$ & 3.0622 & $-\mathrm{DE} / \mathrm{DX}=0.0$ \\
\hline D32 & $\mathrm{D}(15,13,14,17)$ & -178.0922 & $-\mathrm{DE} / \mathrm{DX}=0.0$ \\
\hline D33 & $\mathrm{D}(12,13,15,18)$ & -176.7193 & $-\mathrm{DE} / \mathrm{DX}=$ \\
\hline D34 & $\mathrm{D}(12,13,15,19)$ & 0.2292 & $-\mathrm{DE} / \mathrm{DX}=0.0$ \\
\hline D35 & $\mathrm{D}(14,13,15,18)$ & -1.5779 & $-\mathrm{DE} / \mathrm{DX}=0.0$ \\
\hline D36 & $\mathrm{D}(14,13,15,19)$ & 175.3706 & $-\mathrm{DE} / \mathrm{DX}=0.0$ \\
\hline D37 & $\mathrm{D}(13,14,16,20)$ & -2.2566 & $-\mathrm{DE} / \mathrm{DX}=0.0$ \\
\hline D38 & $\mathrm{D}(13,14,16,21)$ & 178.6333 & $-\mathrm{DE} / \mathrm{DX}=0.0$ \\
\hline D39 & $\mathrm{D}(17,14,16,20)$ & 178.9444 & $-\mathrm{DE} / \mathrm{DX}=$ \\
\hline D40 & $\mathrm{D}(17,14,16,21)$ & -0.1657 & $-\mathrm{DE} / \mathrm{DX}=0.0$ \\
\hline D41 & $\mathrm{D}(13,15,18,20)$ & -0.7027 & $-\mathrm{DE} / \mathrm{DX}=0.0$ \\
\hline D42 & $\mathrm{D}(13,15,18,22)$ & 178.8011 & $-\mathrm{DE} / \mathrm{DX}=0.0$ \\
\hline D43 & $\mathrm{D}(19,15,18,20)$ & -177.6306 & $-\mathrm{DE} / \mathrm{DX}=0.0$ \\
\hline D44 & $\mathrm{D}(19,15,18,22)$ & 1.8732 & $-\mathrm{DE} / \mathrm{DX}=0.0$ \\
\hline D45 & $\mathrm{D}(14,16,20,18)$ & -0.1017 & $-\mathrm{DE} / \mathrm{DX}=0.0$ \\
\hline D46 & $\mathrm{D}(14,16,20,23)$ & -178.9099 & $-\mathrm{DE} / \mathrm{DX}=0.0$ \\
\hline D47 & $\mathrm{D}(21,16,20,18)$ & 179.007 & $-\mathrm{DE} / \mathrm{DX}=0.0$ \\
\hline D48 & $\mathrm{D}(21,16,20,23)$ & 0.1988 & $-\mathrm{DE} / \mathrm{DX}=0.0$ \\
\hline D49 & $\mathrm{D}(15,18,20,16)$ & 1.5796 & $-\mathrm{DE} / \mathrm{DX}=0.0$ \\
\hline D50 & $\mathrm{D}(15,18,20,23)$ & -179.6112 & $-\mathrm{DE} / \mathrm{DX}=0.0$ \\
\hline D51 & $\mathrm{D}(22,18,20,16)$ & -177.9226 & $-\mathrm{DE} / \mathrm{DX}=0.0$ \\
\hline D52 & $\mathrm{D}(22,18,20,23)$ & 0.8866 & $-\mathrm{DE} / \mathrm{DX}=0.0$ \\
\hline
\end{tabular}

CARTESIAN COORDINATES

$\begin{array}{clllll}1 & 6 & 0 & -3.484211 & 1.031111 & -1.281536 \\ 2 & 6 & 0 & -3.095056 & 1.968210 & -.315319 \\ 3 & 6 & 0 & -1.814255 & 1.934807 & .213820 \\ 4 & 6 & 0 & -.887008 & .928755 & -.171894 \\ 5 & 6 & 0 & -1.295287 & -.002410 & -1.168027 \\ 6 & 6 & 0 & -2.571828 & .061213 & -1.712086 \\ 7 & 1 & 0 & -4.481785 & 1.068638 & -1.708496 \\ 8 & 1 & 0 & -3.793911 & 2.733100 & .010633 \\ 9 & 1 & 0 & -1.486742 & 2.661001 & .950406 \\ 10 & 1 & 0 & -.584034 & -.733132 & -1.536148 \\ 11 & 1 & 0 & -2.859470 & -.645537 & -2.485458 \\ 12 & 7 & 0 & .358815 & 1.002544 & .387686 \\ 13 & 6 & 0 & 1.192311 & -.075995 & .501541 \\ 14 & 6 & 0 & 2.580837 & .206726 & .613927 \\ 15 & 6 & 0 & .781071 & -1.434039 & .612856 \\ 16 & 6 & 0 & 3.508832 & -.812348 & .761944 \\ 17 & 1 & 0 & 2.885532 & 1.246644 & .560057 \\ 18 & 6 & 0 & 1.719359 & -2.442697 & .791591 \\ 19 & 1 & 0 & -.276730 & -1.671461 & .606892 \\ 20 & 6 & 0 & 3.085490 & -2.145023 & .850857 \\ 21 & 1 & 0 & 4.566739 & -.574174 & .823578 \\ 22 & 1 & 0 & 1.385684 & -3.471517 & .894166 \\ 23 & 1 & 0 & 3.811476 & -2.941220 & .984516 \\ ------------------\end{array}$


S3.8. $\mathrm{Ph}_{2} \mathrm{NH}$

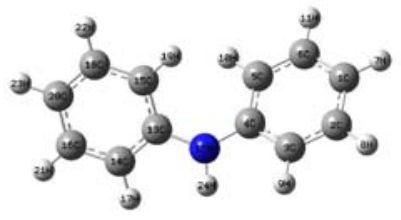

OPTIMIZED PARAMETERS

\begin{tabular}{|c|c|c|c|}
\hline R1 & $\mathrm{R}(1,2)$ & 1.3964 & $-\mathrm{DE} / \mathrm{DX}=0.0$ \\
\hline R2 & $\mathrm{R}(1,6)$ & 1.3954 & $-\mathrm{DE} / \mathrm{DX}=0.0$ \\
\hline R3 & $\mathrm{R}(1,7)$ & 1.0853 & $-\mathrm{DE} / \mathrm{DX}=0.0$ \\
\hline R4 & $\mathrm{R}(2,3)$ & 1.3914 & $-\mathrm{DE} / \mathrm{DX}=0.0$ \\
\hline R5 & $\mathrm{R}(2,8)$ & 1.0864 & $-\mathrm{DE} / \mathrm{DX}=0.0$ \\
\hline R6 & $\mathrm{R}(3,4)$ & 1.407 & $-\mathrm{DE} / \mathrm{DX}=0.0$ \\
\hline R7 & $\mathrm{R}(3,9)$ & 1.0875 & $-\mathrm{DE} / \mathrm{DX}=0.0$ \\
\hline R8 & $\mathrm{R}(4,5)$ & 1.4061 & $-\mathrm{DE} / \mathrm{DX}=0.0$ \\
\hline R9 & $\mathrm{R}(4,12)$ & 1.3987 & $-\mathrm{DE} / \mathrm{DX}=0.0$ \\
\hline R10 & $\mathrm{R}(5,6)$ & 1.3939 & $-\mathrm{DE} / \mathrm{DX}=0.0$ \\
\hline R11 & $\mathrm{R}(5,10)$ & 1.0834 & $-\mathrm{DE} / \mathrm{DX}=0.0$ \\
\hline R12 & $\mathrm{R}(6,11)$ & 1.0865 & $-\mathrm{DE} / \mathrm{DX}=0.0$ \\
\hline R13 & $\mathrm{R}(12,13)$ & 1.3987 & $-\mathrm{DE} / \mathrm{DX}=0.0$ \\
\hline R14 & $\mathrm{R}(12,24)$ & 1.0087 & $-\mathrm{DE} / \mathrm{DX}=0.0$ \\
\hline R15 & $\mathrm{R}(13,14)$ & 1.407 & $-\mathrm{DE} / \mathrm{DX}=0.0$ \\
\hline R16 & $\mathrm{R}(13,15)$ & 1.4061 & $-\mathrm{DE} / \mathrm{DX}=0.0$ \\
\hline R17 & $\mathrm{R}(14,16)$ & 1.3914 & $-\mathrm{DE} / \mathrm{DX}=0.0$ \\
\hline R18 & $\mathrm{R}(14,17)$ & 1.0875 & $-\mathrm{DE} / \mathrm{DX}=0.0$ \\
\hline R19 & $\mathrm{R}(15,18)$ & 1.3939 & $-\mathrm{DE} / \mathrm{DX}=0.0$ \\
\hline R20 & $\mathrm{R}(15,19)$ & 1.0834 & $-\mathrm{DE} / \mathrm{DX}=$ \\
\hline R21 & $\mathrm{R}(16,20)$ & 1.3964 & $-\mathrm{DE} / \mathrm{DX}=0.0$ \\
\hline R22 & $\mathrm{R}(16,21)$ & 1.0864 & $-\mathrm{DE} / \mathrm{DX}=0.0$ \\
\hline R23 & $\mathrm{R}(18,20)$ & 1.3955 & $-\mathrm{DE} / \mathrm{DX}=$ \\
\hline R24 & $\mathrm{R}(18,22)$ & 1.0865 & $-\mathrm{DE} / \mathrm{DX}=$ \\
\hline R25 & $\mathrm{R}(20,23)$ & 1.0853 & $-\mathrm{DE} / \mathrm{DX}=0.0$ \\
\hline A1 & $\mathrm{A}(2,1,6)$ & 118.904 & $-\mathrm{DE} / \mathrm{DX}=0.0$ \\
\hline A2 & $\mathrm{A}(2,1,7)$ & 120.5443 & $-\mathrm{DE} / \mathrm{DX}=0.0$ \\
\hline A3 & $\mathrm{A}(6,1,7)$ & 120.5513 & $-\mathrm{DE} / \mathrm{DX}=0.0$ \\
\hline A4 & $\mathrm{A}(1,2,3)$ & 120.5625 & $-\mathrm{DE} / \mathrm{DX}=0.0$ \\
\hline A5 & $\mathrm{A}(1,2,8)$ & 120.1639 & $-\mathrm{DE} / \mathrm{DX}=0.0$ \\
\hline A6 & $\mathrm{A}(3,2,8)$ & 119.2707 & $-\mathrm{DE} / \mathrm{DX}=0.0$ \\
\hline A7 & $\mathrm{A}(2,3,4)$ & 120.7266 & $-\mathrm{DE} / \mathrm{DX}=0.0$ \\
\hline A8 & $\mathrm{A}(2,3,9)$ & 120.0655 & $-\mathrm{DE} / \mathrm{DX}=0.0$ \\
\hline A9 & $\mathrm{A}(4,3,9)$ & 119.2068 & $-\mathrm{DE} / \mathrm{DX}=0.0$ \\
\hline A10 & $\mathrm{A}(3,4,5)$ & 118.5754 & $-\mathrm{DE} / \mathrm{DX}=0.0$ \\
\hline A11 & $\mathrm{A}(3,4,12)$ & 118.2994 & $-\mathrm{DE} / \mathrm{DX}=0.0$ \\
\hline A12 & $\mathrm{A}(5,4,12)$ & 123.0707 & $-\mathrm{DE} / \mathrm{DX}=0.0$ \\
\hline A13 & $\mathrm{A}(4,5,6)$ & 120.106 & $-\mathrm{DE} / \mathrm{DX}=0.0$ \\
\hline A14 & $\mathrm{A}(4,5,10)$ & 119.9527 & $-\mathrm{DE} / \mathrm{DX}=0.0$ \\
\hline A15 & $\mathrm{A}(6,5,10)$ & 119.9235 & $-\mathrm{DE} / \mathrm{DX}=0.0$ \\
\hline A16 & $\mathrm{A}(1,6,5)$ & 121.113 & $-\mathrm{DE} / \mathrm{DX}=0.0$ \\
\hline A17 & $\mathrm{A}(1,6,11)$ & 119.9561 & $-\mathrm{DE} / \mathrm{DX}=0.0$ \\
\hline A18 & $\mathrm{A}(5,6,11)$ & 118.924 & $-\mathrm{DE} / \mathrm{DX}=0.0$ \\
\hline A19 & $\mathrm{A}(4,12,13)$ & 129.8393 & $-\mathrm{DE} / \mathrm{DX}=0.0$ \\
\hline $\mathrm{A} 20$ & $\mathrm{~A}(4,12,24)$ & 115.0799 & $-\mathrm{DE} / \mathrm{DX}=0.0$ \\
\hline A21 & $\mathrm{A}(13,12,24)$ & 115.0808 & $-\mathrm{DE} / \mathrm{DX}=0.0$ \\
\hline A22 & $\mathrm{A}(12,13,14)$ & 118.3023 & $-\mathrm{DE} / \mathrm{DX}=0.0$ \\
\hline A23 & $\mathrm{A}(12,13,15)$ & 123.0678 & $-\mathrm{DE} / \mathrm{DX}=0.0$ \\
\hline A24 & $\mathrm{A}(14,13,15)$ & 118.5752 & $-\mathrm{DE} / \mathrm{DX}=0.0$ \\
\hline A25 & $\mathrm{A}(13,14,16)$ & 120.7266 & $-\mathrm{DE} / \mathrm{DX}=$ \\
\hline A26 & $\mathrm{A}(13,14,17)$ & 119.2069 & $-\mathrm{DE} / \mathrm{DX}=0.0$ \\
\hline A27 & $\mathrm{A}(16,14,17)$ & 120.0653 & $-\mathrm{DE} / \mathrm{DX}=0.0$ \\
\hline A28 & $\mathrm{A}(13,15,18)$ & 120.1063 & $-\mathrm{DE} / \mathrm{DX}=0.0$ \\
\hline A29 & $\mathrm{A}(13,15,19)$ & 119.9517 & $-\mathrm{DE} / \mathrm{DX}=0.0$ \\
\hline A30 & $\mathrm{A}(18,15,19)$ & 119.9241 & $-\mathrm{DE} / \mathrm{DX}=$ \\
\hline A31 & $\mathrm{A}(14,16,20)$ & 120.5625 & $-\mathrm{DE} / \mathrm{DX}=0.0$ \\
\hline A32 & $\mathrm{A}(14,16,21)$ & 119.2705 & $-\mathrm{DE} / \mathrm{DX}=0.0$ \\
\hline A33 & $\mathrm{A}(20,16,21)$ & 120.1641 & $-\mathrm{DE} / \mathrm{DX}=$ \\
\hline A34 & $\mathrm{A}(15,18,20)$ & 121.1127 & $-\mathrm{DE} / \mathrm{DX}=0.0$ \\
\hline A35 & $\mathrm{A}(15,18,22)$ & 118.9241 & $-\mathrm{DE} / \mathrm{DX}=0.0$ \\
\hline A36 & $\mathrm{A}(20,18,22)$ & 119.9563 & $-\mathrm{DE} / \mathrm{DX}=0.0$ \\
\hline A37 & $\mathrm{A}(16,20,18)$ & 118.9042 & $-\mathrm{DE} / \mathrm{DX}=0.0$ \\
\hline A38 & $\mathrm{A}(16,20,23)$ & 120.5442 & $-\mathrm{DE} / \mathrm{DX}=0.0$ \\
\hline A39 & $\mathrm{A}(18,20,23)$ & 120.551 & $-\mathrm{DE} / \mathrm{DX}=0.0$ \\
\hline D1 & $\mathrm{D}(6,1,2,3)$ & -0.8941 & $-\mathrm{DE} / \mathrm{DX}=0.0$ \\
\hline D2 & $\mathrm{D}(6,1,2,8)$ & 179.7324 & $-\mathrm{DE} / \mathrm{DX}=0.0$ \\
\hline D3 & $\mathrm{D}(7,1,2,3)$ & 179.3632 & $-\mathrm{DE} / \mathrm{DX}=0.0$ \\
\hline D4 & $\mathrm{D}(7,1,2,8)$ & -0.0103 & $-\mathrm{DE} / \mathrm{DX}=0.0$ \\
\hline
\end{tabular}

\begin{tabular}{|c|c|c|c|}
\hline D5 & $\mathrm{D}(2,1,6,5)$ & 0.0756 & $-\mathrm{DE} / \mathrm{DX}=0.0$ \\
\hline D6 & $\mathrm{D}(2,1,6,11)$ & -178.9614 & $-\mathrm{DE} / \mathrm{DX}=0.0$ \\
\hline D7 & $\mathrm{D}(7,1,6,5)$ & 179.8183 & $-\mathrm{DE} / \mathrm{DX}=0.0$ \\
\hline D8 & $\mathrm{D}(7,1,6,11)$ & 0.7812 & $-\mathrm{DE} / \mathrm{DX}=0.0$ \\
\hline D9 & $\mathrm{D}(1,2,3,4)$ & 0.7361 & $-\mathrm{DE} / \mathrm{DX}=0.0$ \\
\hline D10 & $\mathrm{D}(1,2,3,9)$ & -178.8671 & $-\mathrm{DE} / \mathrm{DX}=0.0$ \\
\hline D11 & $\mathrm{D}(8,2,3,4)$ & -179.8849 & $-\mathrm{DE} / \mathrm{DX}=0.0$ \\
\hline D12 & $\mathrm{D}(8,2,3,9)$ & 0.512 & $-\mathrm{DE} / \mathrm{DX}=0.0$ \\
\hline D13 & $\mathrm{D}(2,3,4,5)$ & 0.2439 & $-\mathrm{DE} / \mathrm{DX}=0.0$ \\
\hline D14 & $\mathrm{D}(2,3,4,12)$ & 177.6423 & $-\mathrm{DE} / \mathrm{DX}=0.0$ \\
\hline D15 & $\mathrm{D}(9,3,4,5)$ & 179.8504 & $-\mathrm{DE} / \mathrm{DX}=0.0$ \\
\hline D16 & $\mathrm{D}(9,3,4,12)$ & -2.7512 & $-\mathrm{DE} / \mathrm{DX}=0.0$ \\
\hline D17 & $\mathrm{D}(3,4,5,6)$ & -1.0526 & $-\mathrm{DE} / \mathrm{DX}=0.0$ \\
\hline D18 & $\mathrm{D}(3,4,5,10)$ & 177.4073 & $-\mathrm{DE} / \mathrm{DX}=0.0$ \\
\hline D19 & $\mathrm{D}(12,4,5,6)$ & -178.319 & $-\mathrm{DE} / \mathrm{DX}=0.0$ \\
\hline D20 & $\mathrm{D}(12,4,5,10)$ & 0.1409 & $-\mathrm{DE} / \mathrm{DX}=0.0$ \\
\hline D21 & $\mathrm{D}(3,4,12,13)$ & 157.9359 & $-\mathrm{DE} / \mathrm{DX}=0.0$ \\
\hline D22 & $\mathrm{D}(3,4,12,24)$ & -22.1139 & $-\mathrm{DE} / \mathrm{DX}=0.0$ \\
\hline D23 & $\mathrm{D}(5,4,12,13)$ & -24.7906 & $-\mathrm{DE} / \mathrm{DX}=0.0$ \\
\hline D24 & $\mathrm{D}(5,4,12,24)$ & 155.1596 & $-\mathrm{DE} / \mathrm{DX}=0.0$ \\
\hline D25 & $\mathrm{D}(4,5,6,1)$ & 0.905 & $-\mathrm{DE} / \mathrm{DX}=0.0$ \\
\hline D26 & $\mathrm{D}(4,5,6,11)$ & 179.9518 & $-\mathrm{DE} / \mathrm{DX}=0.0$ \\
\hline D27 & $\mathrm{D}(10,5,6,1)$ & -177.5554 & $-\mathrm{DE} / \mathrm{DX}=0.0$ \\
\hline D28 & $\mathrm{D}(10,5,6,11)$ & 1.4914 & $-\mathrm{DE} / \mathrm{DX}=0.0$ \\
\hline D29 & $\mathrm{D}(4,12,13,14)$ & 157.867 & $-\mathrm{DE} / \mathrm{DX}=0.0$ \\
\hline D30 & $\mathrm{D}(4,12,13,15)$ & -24.8648 & $-\mathrm{DE} / \mathrm{DX}=0.0$ \\
\hline D31 & $\mathrm{D}(24,12,13,14)$ & -22.0831 & $-\mathrm{DE} / \mathrm{DX}=0.0$ \\
\hline D32 & $\mathrm{D}(24,12,13,15)$ & 155.1851 & $-\mathrm{DE} / \mathrm{DX}=0.0$ \\
\hline D33 & $\mathrm{D}(12,13,14,16)$ & 177.6368 & $-\mathrm{DE} / \mathrm{DX}=0.0$ \\
\hline D34 & $\mathrm{D}(12,13,14,17)$ & -2.7566 & $-\mathrm{DE} / \mathrm{DX}=0.0$ \\
\hline D35 & $\mathrm{D}(15,13,14,16)$ & 0.2436 & $-\mathrm{DE} / \mathrm{DX}=0.0$ \\
\hline D36 & $\mathrm{D}(15,13,14,17)$ & 179.8502 & $-\mathrm{DE} / \mathrm{DX}=0.0$ \\
\hline D37 & $\mathrm{D}(12,13,15,18)$ & -178.3142 & $-\mathrm{DE} / \mathrm{DX}=0.0$ \\
\hline D38 & $\mathrm{D}(12,13,15,19)$ & 0.1457 & $-\mathrm{DE} / \mathrm{DX}=0.0$ \\
\hline D39 & $\mathrm{D}(14,13,15,18)$ & -1.0531 & $-\mathrm{DE} / \mathrm{DX}=0.0$ \\
\hline D40 & $\mathrm{D}(14,13,15,19)$ & 177.4068 & $-\mathrm{DE} / \mathrm{DX}=0.0$ \\
\hline D41 & $\mathrm{D}(13,14,16,20)$ & 0.7367 & $-\mathrm{DE} / \mathrm{DX}=0.0$ \\
\hline D42 & $\mathrm{D}(13,14,16,21)$ & -179.8846 & $-\mathrm{DE} / \mathrm{DX}=0.0$ \\
\hline D43 & $\mathrm{D}(17,14,16,20)$ & -178.8665 & $-\mathrm{DE} / \mathrm{DX}=0.0$ \\
\hline D44 & $\mathrm{D}(17,14,16,21)$ & 0.5121 & $-\mathrm{DE} / \mathrm{DX}=0.0$ \\
\hline D45 & $\mathrm{D}(13,15,18,20)$ & 0.906 & $-\mathrm{DE} / \mathrm{DX}=0.0$ \\
\hline D46 & $\mathrm{D}(13,15,18,22)$ & 179.9523 & $-\mathrm{DE} / \mathrm{DX}=0.0$ \\
\hline D47 & $\mathrm{D}(19,15,18,20)$ & -177.5544 & $-\mathrm{DE} / \mathrm{DX}=0.0$ \\
\hline D48 & $\mathrm{D}(19,15,18,22)$ & 1.492 & $-\mathrm{DE} / \mathrm{DX}=0.0$ \\
\hline D49 & $\mathrm{D}(14,16,20,18)$ & -0.8943 & $-\mathrm{DE} / \mathrm{DX}=0.0$ \\
\hline D50 & $\mathrm{D}(14,16,20,23)$ & 179.3632 & $-\mathrm{DE} / \mathrm{DX}=0.0$ \\
\hline D51 & $\mathrm{D}(21,16,20,18)$ & 179.7326 & $-\mathrm{DE} / \mathrm{DX}=0.0$ \\
\hline D52 & $\mathrm{D}(21,16,20,23)$ & -0.01 & $-\mathrm{DE} / \mathrm{DX}=0.0$ \\
\hline D53 & $\mathrm{D}(15,18,20,16)$ & 0.075 & $-\mathrm{DE} / \mathrm{DX}=0.0$ \\
\hline D54 & $\mathrm{D}(15,18,20,23)$ & 179.8176 & $-\mathrm{DE} / \mathrm{DX}=0.0$ \\
\hline D55 & $\mathrm{D}(22,18,20,16)$ & -178.9616 & $-\mathrm{DE} / \mathrm{DX}=0.0$ \\
\hline D56 & $\mathrm{D}(22,18,20,23)$ & 0.781 & $-\mathrm{DE} / \mathrm{DX}=0.0$ \\
\hline
\end{tabular}

CARTESIAN COORDINATES

\begin{tabular}{|c|c|c|c|c|c|}
\hline 1 & 6 & 0 & -3.526775 & 1.096280 & -1.344301 \\
\hline 2 & 6 & 0 & -3.106630 & 2.034469 & -.399196 \\
\hline 3 & 6 & 0 & -1.840648 & 1.945891 & .171278 \\
\hline 4 & 6 & 0 & -.953593 & .919908 & -.203135 \\
\hline 5 & 6 & 0 & -1.371922 & -.011221 & -1.170202 \\
\hline 6 & 6 & 0 & -2.649005 & .079108 & -1.721337 \\
\hline 7 & 1 & 0 & -4.518083 & 1.159685 & -1.781466 \\
\hline 8 & 1 & 0 & -3.771733 & 2.837402 & -.094055 \\
\hline 9 & 1 & 0 & -1.528608 & 2.670140 & .920068 \\
\hline 10 & 1 & 0 & -.691972 & -.785042 & -1.505777 \\
\hline 11 & 1 & 0 & -2.952019 & -.650017 & -2.467687 \\
\hline 12 & 7 & 0 & .324373 & .908081 & .365184 \\
\hline 13 & 6 & 0 & 1.229311 & -.148031 & .513535 \\
\hline 14 & 6 & 0 & 2.592915 & .159439 & .673952 \\
\hline 15 & 6 & 0 & .833219 & -1.496235 & .565419 \\
\hline 16 & 6 & 0 & 3.530284 & -.849084 & .874509 \\
\hline 17 & 1 & 0 & 2.910948 & 1.198566 & .633175 \\
\hline 18 & 6 & 0 & 1.784754 & -2.497893 & .750072 \\
\hline 19 & 1 & 0 & -.215822 & -1.755215 & .486749 \\
\hline 20 & 6 & 0 & 3.136612 & -2.188495 & .905115 \\
\hline 21 & 1 & 0 & 4.576799 & -.584763 & .997455 \\
\hline 22 & 1 & 0 & 1.457326 & -3.533187 & .787984 \\
\hline 23 & 1 & 0 & 3.869420 & -2.975545 & 1.051297 \\
\hline 24 & 1 & 0 & 641993 & 1.796587 & .721721 \\
\hline
\end{tabular}


S3.9. $\mathrm{PhNH}^{\circ}$

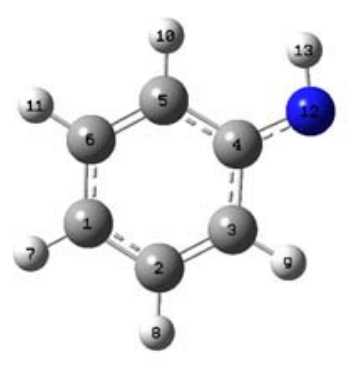

CARTESIAN COORDINATES

$\begin{array}{cccccc}1 & 6 & 0 & -1.538687 & .096521 & -.946256 \\ 2 & 6 & 0 & -1.582003 & .102896 & .458007 \\ 3 & 6 & 0 & -.413084 & .029533 & 1.192401 \\ 4 & 6 & 0 & .862955 & -.054366 & .542882 \\ 5 & 6 & 0 & .873957 & -.058012 & -.892245 \\ 6 & 6 & 0 & -.304472 & .015737 & -1.612411 \\ 7 & 1 & 0 & -2.459689 & .154265 & -1.518226 \\ 8 & 1 & 0 & -2.539588 & .165562 & .966593 \\ 9 & 1 & 0 & -.417773 & .032721 & 2.277381 \\ 10 & 1 & 0 & 1.831071 & -.120429 & -1.404679 \\ 11 & 1 & 0 & -.277694 & .011475 & -2.698292 \\ 12 & 7 & 0 & 1.955203 & -.122841 & 1.315721 \\ 13 & 1 & 0 & 2.785257 & -.177566 & .712906\end{array}$

\section{S3.10. $\mathrm{Ph}_{2} \mathrm{NH}$}

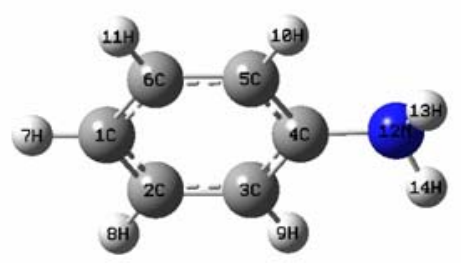

OPTIMIZED PARAMETERS

\begin{tabular}{|c|c|c|c|}
\hline R1 & $\mathrm{R}(1,2)$ & 1.3962 & $-\mathrm{DE} / \mathrm{DX}=$ \\
\hline R2 & $\mathrm{R}(1,6)$ & 1.3962 & $-\mathrm{DE} / \mathrm{DX}=$ \\
\hline R3 & $\mathrm{R}(1,7)$ & 1.0853 & $-\mathrm{DE} / \mathrm{DX}=$ \\
\hline R4 & $\mathrm{R}(2,3)$ & 1.3928 & $-\mathrm{DE} / \mathrm{DX}=$ \\
\hline R5 & $\mathrm{R}(2,8)$ & 1.0866 & $-\mathrm{DE} / \mathrm{DX}=$ \\
\hline R6 & $\mathrm{R}(3,4)$ & 1.4054 & $-\mathrm{DE} / \mathrm{DX}=$ \\
\hline R7 & $\mathrm{R}(3,9)$ & 1.0875 & $-\mathrm{DE} / \mathrm{DX}=$ \\
\hline R8 & $\mathrm{R}(4,5)$ & 1.4053 & $-\mathrm{DE} / \mathrm{DX}=$ \\
\hline R9 & $\mathrm{R}(4,12)$ & 1.398 & $-\mathrm{DE} / \mathrm{DX}=0.0001$ \\
\hline R10 & $\mathrm{R}(5,6)$ & 1.3928 & $-\mathrm{DE} / \mathrm{DX}=0.0$ \\
\hline R11 & $\mathrm{R}(5,10)$ & 1.0875 & $-\mathrm{DE} / \mathrm{DX}=0.0$ \\
\hline R12 & $\mathrm{R}(6,11)$ & 1.0866 & $-\mathrm{DE} / \mathrm{DX}=0.0$ \\
\hline R13 & $\mathrm{R}(12,13)$ & 1.011 & $-\mathrm{DE} / \mathrm{DX}=$ \\
\hline R14 & $\mathrm{R}(12,14)$ & 1.011 & $-\mathrm{DE} / \mathrm{DX}=0.0$ \\
\hline A1 & $\mathrm{A}(2,1,6)$ & 118.8885 & $-\mathrm{DE} / \mathrm{DX}=0.0$ \\
\hline A2 & $\mathrm{A}(2,1,7)$ & 120.5524 & $-\mathrm{DE} / \mathrm{DX}=$ \\
\hline A3 & $\mathrm{A}(6,1,7)$ & 120.5591 & $-\mathrm{DE} / \mathrm{DX}=0.0$ \\
\hline A4 & $\mathrm{A}(1,2,3)$ & 120.7949 & $-\mathrm{DE} / \mathrm{DX}=0.0$ \\
\hline A5 & $\mathrm{A}(1,2,8)$ & 120.0192 & $-\mathrm{DE} / \mathrm{DX}=$ \\
\hline A6 & $\mathrm{A}(3,2,8)$ & 119.1857 & $-\mathrm{DE} / \mathrm{DX}=0.0$ \\
\hline A7 & $\mathrm{A}(2,3,4)$ & 120.4763 & $-\mathrm{DE} / \mathrm{DX}=0.0$ \\
\hline A8 & $\mathrm{A}(2,3,9)$ & 120.1177 & $-\mathrm{DE} / \mathrm{DX}=0.0$ \\
\hline A9 & $\mathrm{A}(4,3,9)$ & 119.4054 & $-\mathrm{DE} / \mathrm{DX}=0.0$ \\
\hline A10 & $\mathrm{A}(3,4,5)$ & 118.5692 & $-\mathrm{DE} / \mathrm{DX}=0.0$ \\
\hline A11 & $\mathrm{A}(3,4,12)$ & 120.6778 & $-\mathrm{DE} / \mathrm{DX}=0.0$ \\
\hline A12 & $\mathrm{A}(5,4,12)$ & 120.6937 & $-\mathrm{DE} / \mathrm{DX}=0.0$ \\
\hline A13 & $\mathrm{A}(4,5,6)$ & 120.4734 & $-\mathrm{DE} / \mathrm{DX}=0.0$ \\
\hline A14 & $\mathrm{A}(4,5,10)$ & 119.4222 & $-\mathrm{DE} / \mathrm{DX}=0.0$ \\
\hline A15 & $\mathrm{A}(6,5,10)$ & 120.1039 & $-\mathrm{DE} / \mathrm{DX}=0.0$ \\
\hline A16 & $\mathrm{A}(1,6,5)$ & 120.7977 & $-\mathrm{DE} / \mathrm{DX}=0.0$ \\
\hline A17 & $\mathrm{A}(1,6,11)$ & 120.0251 & $-\mathrm{DE} / \mathrm{DX}=0.0$ \\
\hline A18 & $\mathrm{A}(5,6,11)$ & 119.1771 & $-\mathrm{DE} / \mathrm{DX}=0.0$ \\
\hline A19 & $\mathrm{A}(4,12,13)$ & 114.8678 & $-\mathrm{DE} / \mathrm{DX}=0.0$ \\
\hline A20 & $\mathrm{A}(4,12,14)$ & 114.8656 & $-\mathrm{DE} / \mathrm{DX}=0.0$ \\
\hline A21 & $\mathrm{A}(13,12,14)$ & 111.5297 & $-\mathrm{DE} / \mathrm{DX}=0.0$ \\
\hline D1 & $\mathrm{D}(6,1,2,3)$ & 0.0538 & $-\mathrm{DE} / \mathrm{DX}=0.0$ \\
\hline D2 & $\mathrm{D}(6,1,2,8)$ & 179.8967 & $-\mathrm{DE} / \mathrm{DX}=0.0$ \\
\hline D3 & $\mathrm{D}(7,1,2,3)$ & -179.8992 & $-\mathrm{DE} / \mathrm{DX}=0.0$ \\
\hline D4 & $\mathrm{D}(7,1,2,8)$ & -0.0563 & $-\mathrm{DE} / \mathrm{DX}=0.0$ \\
\hline D5 & $\mathrm{D}(2,1,6,5)$ & -0.0548 & $-\mathrm{DE} / \mathrm{DX}=0.0$ \\
\hline D6 & $\mathrm{D}(2,1,6,11)$ & -179.9016 & $-\mathrm{DE} / \mathrm{DX}=0.0$ \\
\hline D7 & $\mathrm{D}(7,1,6,5)$ & 179.8982 & $-\mathrm{DE} / \mathrm{DX}=0.0$ \\
\hline D8 & $\mathrm{D}(7,1,6,11)$ & 0.0514 & $-\mathrm{DE} / \mathrm{DX}=0.0$ \\
\hline D9 & $\mathrm{D}(1,2,3,4)$ & -0.0372 & $-\mathrm{DE} / \mathrm{DX}=0.0$ \\
\hline D10 & $\mathrm{D}(1,2,3,9)$ & -179.7489 & $-\mathrm{DE} / \mathrm{DX}=0.0$ \\
\hline D11 & $\mathrm{D}(8,2,3,4)$ & -179.8814 & $-\mathrm{DE} / \mathrm{DX}=0.0$ \\
\hline
\end{tabular}




\begin{tabular}{|c|c|c|c|}
\hline D12 & $\mathrm{D}(8,2,3,9)$ & 0.4069 & $-\mathrm{DE} / \mathrm{DX}=$ \\
\hline D13 & $\mathrm{D}(2,3,4,5)$ & 0.0203 & $-\mathrm{DE} / \mathrm{DX}=0.0$ \\
\hline D14 & $\mathrm{D}(2,3,4,12)$ & -177.1995 & $-\mathrm{DE} / \mathrm{DX}=0.0$ \\
\hline D15 & $\mathrm{D}(9,3,4,5)$ & 179.734 & $-\mathrm{DE} / \mathrm{DX}=0.0$ \\
\hline D16 & $\mathrm{D}(9,3,4,12)$ & 2.5143 & $-\mathrm{DE} / \mathrm{DX}=0.0$ \\
\hline D17 & $\mathrm{D}(3,4,5,6)$ & -0.0213 & $-\mathrm{DE} / \mathrm{DX}=0.0$ \\
\hline D18 & $\mathrm{D}(3,4,5,10)$ & -179.7505 & $-\mathrm{DE} / \mathrm{DX}=0.0$ \\
\hline D19 & $\mathrm{D}(12,4,5,6)$ & 177.198 & $-\mathrm{DE} / \mathrm{DX}=0.0$ \\
\hline D20 & $\mathrm{D}(12,4,5,10)$ & -2.5312 & $-\mathrm{DE} / \mathrm{DX}=0.0$ \\
\hline D21 & $\mathrm{D}(3,4,12,13)$ & -157.1342 & $-\mathrm{DE} / \mathrm{DX}=0.0$ \\
\hline D22 & $\mathrm{D}(3,4,12,14)$ & -25.7864 & $-\mathrm{DE} / \mathrm{DX}=0.0$ \\
\hline D23 & $\mathrm{D}(5,4,12,13)$ & 25.7054 & $-\mathrm{DE} / \mathrm{DX}=0.0$ \\
\hline D24 & $\mathrm{D}(5,4,12,14)$ & 157.0531 & $-\mathrm{DE} / \mathrm{DX}=0.0$ \\
\hline D25 & $\mathrm{D}(4,5,6,1)$ & 0.0392 & $-\mathrm{DE} / \mathrm{DX}=0.0$ \\
\hline D26 & $\mathrm{D}(4,5,6,11)$ & 179.8873 & $-\mathrm{DE} / \mathrm{DX}=0.0$ \\
\hline D27 & $\mathrm{D}(10,5,6,1)$ & 179.7666 & $-\mathrm{DE} / \mathrm{DX}=0.0$ \\
\hline D28 & $\mathrm{D}(10,5,6,11)$ & -0.3853 & $-\mathrm{DE} / \mathrm{DX}=0.0$ \\
\hline
\end{tabular}

\begin{tabular}{|c|c|c|c|c|c|}
\hline \multicolumn{6}{|c|}{ CARTESIAN COORDINATES } \\
\hline 1 & 6 & 0 & -1.660902 & .043358 & -.882376 \\
\hline 2 & 6 & 0 & -1.594662 & .121447 & .510092 \\
\hline 3 & 6 & 0 & -.367869 & .087453 & 1.168623 \\
\hline 4 & 6 & 0 & .828858 & -.027207 & .440795 \\
\hline 5 & 6 & 0 & .758263 & -.105908 & -.960547 \\
\hline 6 & 6 & 0 & -.473995 & -.070998 & -1.608779 \\
\hline 7 & 1 & 0 & -2.618895 & .071851 & -1.391569 \\
\hline 8 & 1 & 0 & -2.506851 & .212517 & 1.093461 \\
\hline 9 & 1 & 0 & -.329337 & .144955 & 2.253893 \\
\hline 10 & 1 & 0 & 1.675515 & -.199047 & -1.537260 \\
\hline 11 & 1 & 0 & -.503948 & -.131499 & -2.693279 \\
\hline 12 & 7 & 0 & 2.058278 & -.120288 & 1.099705 \\
\hline 13 & 1 & 0 & 2.858301 & .159703 & .548581 \\
\hline 14 & 1 & 0 & 2.079113 & 294666 & 2.021387 \\
\hline
\end{tabular}

\section{REFERENCES}

\section{Complete reference M. J. Frisch et al.:}

GAUSSIAN03, Revision B.05, M. J. Frisch, G. W. Trucks, H. B. Schlegel, G. E. Scuseria, M. A. Robb, J. R. Cheeseman, J. A. Montgomery, Jr., T. Vreven, K. N. Kudin, J. C. Burant, J. M. Millam, S. S. Iyengar, J. Tomasi, V. Barone, B. Mennucci, M. Cossi, G. Scalmani, N. Rega, G. A. Petersson, H. Nakatsuji, M. Hada, M. Ehara, K. Toyota, R. Fukuda, J. Hasegawa, M. Ishida, T. Nakajima, Y. Honda, O. Kitao, H. Nakai, M. Klene, X. Li, J. E. Knox, H. P. Hratchian, J. B. Cross, V. Bakken, C. Adamo, J. Jaramillo, R. Gomperts, R. E. Stratmann, O. Yazyev, A. J. Austin, R. Cammi, C. Pomelli, J. W. Ochterski, P. Y. Ayala, K. Morokuma, G. A. Voth, P. Salvador, J. J. Dannenberg, V. G. Zakrzewski, S. Dapprich, A. D. Daniels, M. C. Strain, O. Farkas, D. K. Malick, A. D. Rabuck, K. Raghavachari, J. B. Foresman, J. V. Ortiz, Q. Cui, A. G. Baboul, S. Clifford, J. Cioslowski, B. B. Stefanov, G. Liu, A. Liashenko, P. Piskorz, I. Komaromi, R. L. Martin, D. J. Fox, T. Keith, M. A. Al-Laham, C. Y. Peng, A. Nanayakkara, M. Challacombe, P. M. W. Gill, B. Johnson, W. Chen, M. W. Wong, C. Gonzalez, and J. A. Pople, Gaussian, Inc., Wallingford CT, 2004. 